\title{
Identification of new OPA1 cleavage site reveals that short isoforms regulate mitochondrial fusion
}

\author{
Thesis By \\ Ruohan Wang
}

In Partial Fulfillment of the Requirements for the Degree of

Doctor of Philosophy

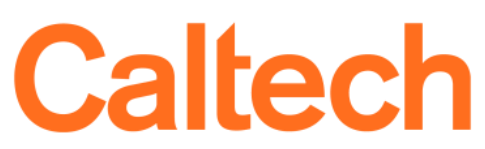

California Institute of Technology

Pasadena, CA

2021

Defended Oct 28, 2020 
(C)2021

Ruohan Wang All Rights Reserved 


\section{Acknowledgement}

I would like to thank my advisor, Dr. David Chan, for his guidance and support for me throughout my graduate training. Monday afternoon meetings have been filled with insightful discussions and kept me on the right track constantly. I would also like to express special thanks to Dr. Hsiuchen Chen, who not only provided insights during lab meetings, but also a good friend to chat with. I will not forget all the random topics we discussed across the bench, from travel, education, TV shows to latest news.

I want to thank the members of my committee, Professor Marianne Bronner, Professor Alex Van Der Bliek, Professor Alexei Aravin and Professor Rebecca Voorhees, for their advice and support. It has been quite a delight for me gathering together the brightest scientists into one tiny room/zoom meeting and discuss the project I worked on. Special thanks to Professor Van der Bliek, who not only drove from UCLA all the way down to Pasadena and helped me through my candidacy meeting, but also gave me enormous courage. I was honored to be greatly encouraged by an expert in the field to continue my research even back in second year.

I am grateful for both the past and present members of the Chan lab_-Dr. Prashant Mishra, who was my mentor when I joined and provided me first hand guidance on my very first project; Dr. Chunshik Shin, for being the best bay mate and always ready for my cell culture questions; Dr. Rebecca Rojansky, for being a good friend and rotation mentor; Dr. Raymond Liu, for always being encouraging and willing to help; Dr. Greg Varuzhanyan, for bringing in various Armenian desserts that I have never seen; Dr. Yoga Chakraborty, for being cheerful and so ready 
to help; Dr. Chris Fiorese for the cheerful laughter and meaningful discussions; Shuxia Meng, for being the best lab manager and making the work environment convenient and smooth.

I have my deepest gratitude for my parents, who not only raised me with enormous love and care, but also dedicated all they have to my education. My mother and father have always been great mentors to me as well as close friends, and I hope that I have become the daughter that they are proud of.

My friend, Yi Jin, have provided me so much support, company and love. Located on the opposite coast of the country, our friendship became tighter despite distance.

Finally, I would like to thank Wei-Hsiang Lin, whose love, humor, support, and wisdom have accompanied my entire graduate study. He has always been there for me, good or bad, ups and downs, during this precious period of my time. My days with him have kept me positive and happy throughout the years. 


\begin{abstract}
OPA1 is a $120 \mathrm{kDa}$ large GTPase belonging to the dynamin superfamily. It is the only known mitochondrial inner membrane fusion protein, mediating fusion of the mitochondrial inner membranes following outer membrane fusion. Additionally, OPA1 also regulates cristae morphology and maintains respiratory chain function.

OPA1 has two forms - inner-membrane-anchored long forms (1-OPA1) and cleaved intermembrane-space only short forms (s-OPA1). L-OPA1 are proteolytically processed by two mitochondrial proteases-OMA1 and YME1L, acting at cleavage sites S1 and S2 respectively, to produce s-OPA1.

In both mice and human, half of the mRNA splice forms of Opal are constitutively processed post translation to yield exclusively s-OPA1. However, the specific function of s-OPA1 in mitochondrial fusion has been debated — under basal conditions, s-OPA1 are needed to maintain optimal fusion activity, but in certain stress conditions, s-OPA1 is dispensable for fusion. By constructing cells in which the Opal locus no longer produces transcripts with S2 cleavage sites using CRISPR-Cas9, we generated a simplified system to identify the novel YME1L-dependent site S3 that mediates constitutive and complete cleavage of OPA1. We found that S3 site locates within the C-terminal leucine string of Opal exon4b, slightly upstream of the well-established S1. We show that mitochondrial morphology is highly sensitive to the ratio of 1-OPA1 to s-OPA1, indicating that s-OPA1 fine tunes mitochondrial fusion.
\end{abstract}




\section{Published Content and Contributions}

Wang, R., Mishra P., Garbis, S., Moradian, A., Sweredoski, M., and Chan, D.C. (2020).

Identification of new OPA1 cleavage site reveals that short isoforms regulate mitochondrial

fusion. Mol Biol Cell. doi: 10.1091/mbc.E20-09-0605

RW and DCC conceptualized this project, analyzed the data, and wrote the manuscript. RW carried the experimental analysis. PM performed the PA-GFP fusion assays. AM performed the LC-MS experiments, and the data were analyzed by SDG and MJS 


\section{Table of Contents}

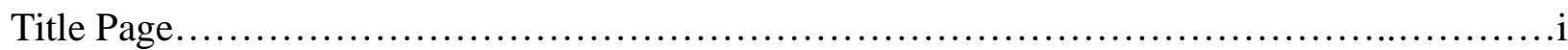

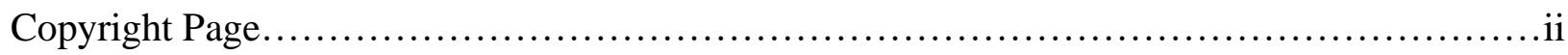

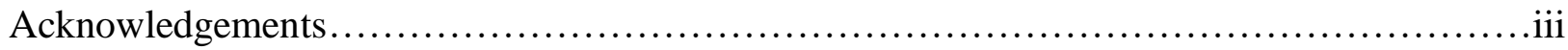

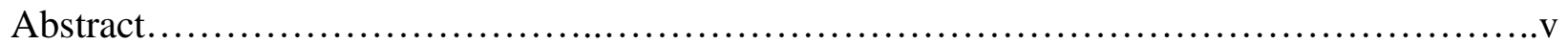

Published Content and Contributions.....................................................

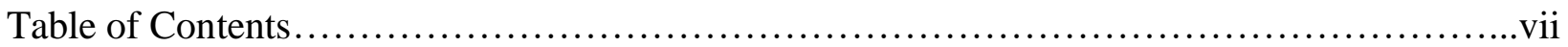

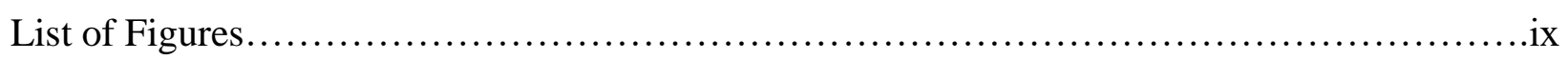

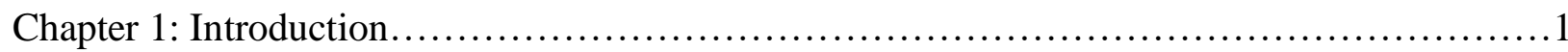

Mitochondria Overview......................................................

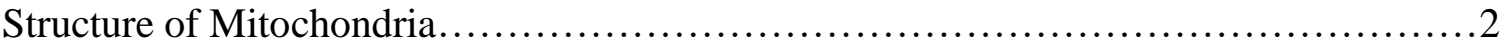

Mitochondrial Dynamics.........................................................

Mitochondria Transport and Turnover.......................................4

Mitochondrial Fission........................................................

Mitochondrial Fusion......................................................

Molecular Machinery of Mitochondrial Fusion...............................10

Outer Membrane Fusion (MFN1\&2) …................................ 10

Inner Membrane Fusion (OPA1) ..........................................12

Physiological Roles of Mitochondrial Fusion...................................19

Mitochondrial Morphology Regulation.............................................19

Inter-mitochondria Content Exchange Facilitation.................................20

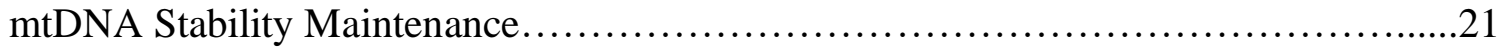

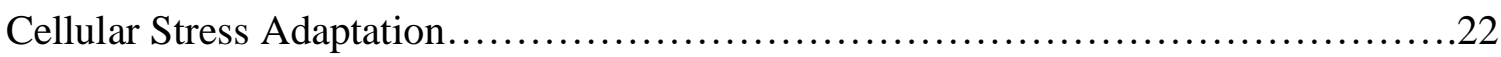

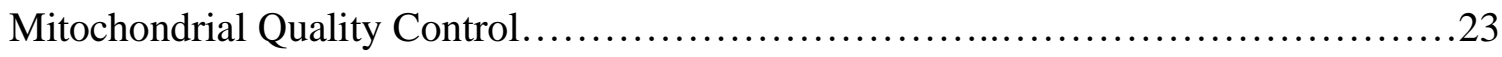


Outline of Thesis

References......

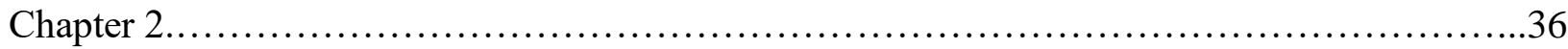

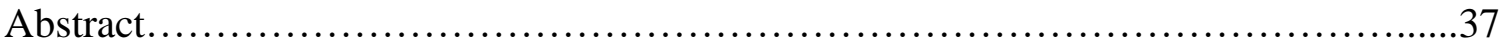

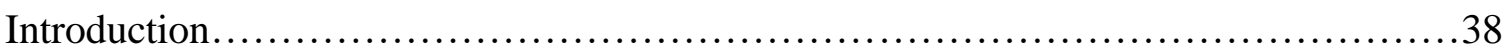

Results......................................................................... 41

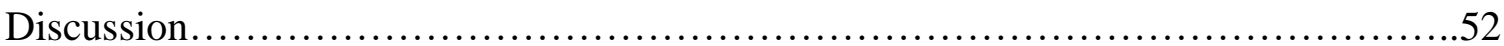

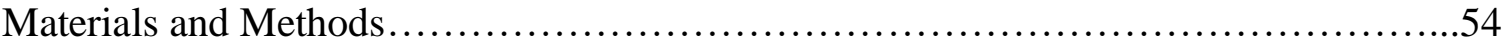

Acknowledgements.........................................................6

Figure Legends.......................................................61

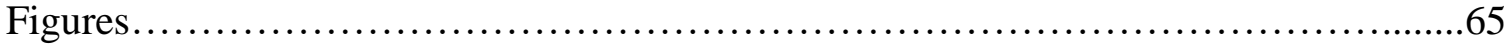

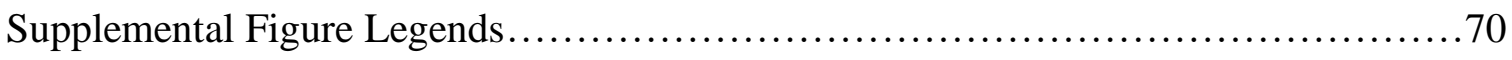

Supplemental Figures....................................................... 73

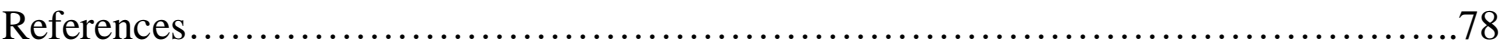

Chapter 3: Discussion....................................................... 87

Reference................................................................ 95 


\section{List of Figures}

\section{Chapter 1}

Fig 1.1 Mitochondria Cristae structure

.25

Fig 1.2 Human OPA1 isoforms overview .26

Chapter 2

Fig 2.1 OMA1 and YME1L differentially regulate OPA1 fusion activity

Fig 2.2 The S2 site encoded by Opa1 exon 5b is necessary for OXPHOS-induced fusion in vivo .66

Fig 2.3 Detection of a new OPA1 cleavage in WT and $\Delta$ exon $5 \mathrm{~b}$ cells.... .67

Fig 2.4 Identification of the YME1L-dependent OPA1 S3 cleavage site within exon 4b. .68

Fig 2.5 The S3-cleaved short form of isoform 5 regulates mitochondrial morphology.... 69

Fig S2.1 OPA1 mRNA isoforms and the role of Oma1 .73

Fig S2.2 Phenotypic characterization of $\triangle$ exon $5 \mathrm{~b}$ MEFs and mice. . .74

Fig S2.3 shRNA knockdown efficiency and primer design for Opa1 mRNA identification.....75

Fig S2.4 Tubulation of mitochondria by isoform 5 mutants.... .76

Fig S2.5 Short form of isoform 5 regulates mitochondrial morphology.... .77 


\section{Chapter 1}

\section{Introduction}

\section{Mitochondria Overview}

The mitochondrion is an organelle of bacterial ancestry, originating from within the bacterial phylum $\alpha$-Proteobacteria (Alphaproteobacteria). The well-known endosymbiotic hypothesis states that the first mitochondrion evolved from a bacterial progenitor via symbiosis with a proto-eukaryotic host cell. The strongest evidence for this hypothesis comes from phylogenetic gene analysis, including both mitochondrial DNAs and ribosomal RNAs, which show homology to the $\alpha$-Proteobacteria. The mitochondrial genome contains in total 37 genes. 13 of the 37 genes encode proteins; 2 encode ribosomal RNAs; and 22 encode transfer RNAs. All of the 37 genes are essential for OXPHOS activity and high levels of mtDNA mutations generally result in impaired energy generation (Carelli and Chan, 2014). Yet mitochondrial genome evolution has taken radically different pathways in diverse eukaryotic lineages, and the organelle itself is increasingly viewed as a genetic and functional mosaic, with the bulk of the mitochondrial proteome having an evolutionary origin outside Alphaproteobacteria. The vast majority of the $\sim 1,000$ proteins of the mitochondrial proteome are encoded in the nuclear genome, synthesized in the cytosol, and imported into the organelle via rigorous importing systems on mitochondrial membranes.

Mitochondria fulfill several essential functions in eukaryotic cells. Long been described as the powerhouses for generating ATP via oxidative phosphorylation, they also play a central role in $\mathrm{Ca} 2+$ signaling, lipids metabolism, apoptosis and innate immunity. They can also produce 
damaging metabolic byproducts such as ammonia and reactive oxygen species (ROS) sequestration (Chan, 2020). In addition, mitochondria are now understood to play active roles in integrating signaling pathways and responses to stressors (Focusing on mitochondrial form and function, 2018). Due to these significant physiological functions, with a few exceptions, mitochondria are found in all eukaryotic cells with varying numbers per cell among different organisms and tissue types. In mammals, except for mature human erythrocytes, mitochondria are utilized by every single cell to meet the varying energetic demands of a specific tissue, ranging from $\sim 80$ to $\sim 2000$ per cell (Cole, 2016). These demands are great in tissues such as the heart and brain, where a dysfunction of mitochondria has been associated with an increasing number of human diseases. Mitochondrial activity must therefore be tightly monitored and optimized to meet any changes in cellular metabolism and physiology.

\section{Structure of mitochondria}

Mitochondria are double-membrane organelles. The outer membrane (OM) is a lipid bilayer membrane separating mitochondria compartments apart from the cytosol. The OM is porous and freely traversed by ions and small, uncharged molecules through pore-forming membrane proteins (porins), such as the voltage-dependent anion channel VDAC (Bayrhuber et al., 2008). Any larger molecules, especially proteins, have to be imported by special translocases. Because of its porosity, there is no membrane potential across the outer membrane.

In contrast, the inner membrane is a tight diffusion barrier to all ions and molecules. Substrates waiting to get inside the mitochondrial matrix can only get across in the presence of corresponding membrane transport proteins, each of which is selective for a particular ion or 
molecule. As a result of the ion selectivity of the inner membrane, an electrochemical membrane potential of about $180 \mathrm{mV}$ builds up across the inner mitochondrial membrane (Kühlbrandt, 2015). Oxidative phosphorylation (OXPHOS) takes place on the inner membrane through a series of biochemical processes in the respiratory complexes. More specifically, in a chain of membrane protein complexes, electrons are transferred across different compartments and protons get pumped out into the inter membrane space, creating the electrochemical gradient across the inner membrane and to the ATP synthase. One of the most functionally important part of the IM is the cristae (Fig 1.1), the invaginated membrane harboring membrane proteins, such as the electron transport chain (ETC) complexes. Cristae can be lamellar, likely formed by infoldings of the IM sheet, or tubular (Riva et al., 2005; Hoppel et al., 2009). The intra-cristal space (ICS) is the inside space of lamellar or tubular cristae, continuous with the IMS; however, the functional continuity is thought to be limited by the narrow cristae junction (CJ) structure. The rest of the IM that runs parallel with the OM is the inner boundary membrane (IBM). Recent studies have shown that the cristae structure is also dynamically regulated and constantly remodeled within individual mitochondria, with the functional regulation of MICOS complex (Kondadi et al., 2020).

\section{Mitochondrial Dynamics}

How do cells maintain their healthy mitochondrial networks? One of the fundamental ways is through the versatile regulation mechanisms of mitochondrial morphology and dynamics. The term mitochondrial dynamics encompasses the processes of mitochondrial fusion, fission, turnover, and transport. 
Mitochondria are remarkably dynamic organelles. Individual mitochondria can have a variety of morphologies, appearing as small spheres, short or long tubules, or interconnected tubules. These morphologies are controlled by the opposing processes of fusion and fission. More specifically, during fusion events, two or more mitochondria fuse together into rods or chains and further threads, which later may form a connected network. During fission events, a part of tubular mitochondria gets constricted and later divide into two or more smaller mitochondria spheres. As discussed previously, mitochondria have dual membranes; therefore, mitochondrial membrane dynamics is a complicated multistep process that requires the coordinated regulation of protein and lipid molecules on both membranes. A fibroblast can have a population of dozens to hundreds of mitochondria, each containing at least one mtDNA genome.

\section{Mitochondria Transport and Turnover}

Mitochondria are distributed across the cell through cytoskeletons, most times along microtubules in mammalian cells. There is a well-designed transport machinery involving various protein motors and adaptors responsible for anchoring and moving mitochondria along the microtubule tracks. Due to the special shape of neurons and the need to transport mitochondria across neuronal axons and dendrites, mitochondria trafficking has been especially widely studied in neurons. Trafficking of mitochondria is bi-directional-anterograde transport towards microtubule plus end from cell soma to dendrites and axons, and retrograde transport towards microtubule minus end from synapses/dendrites back to soma. First discovered in Drosophila, two different motor proteins are used in the opposing transport systemsanterograde transport utilizing Kinesin-1 and retrograde transport utilizing Dynein (Pilling et al., 2006). The attachment of microtubule motor proteins to mitochondria is mediated by 
mitochondrial outer membrane proteins RhoT (small Rho GTPase, in mammalian cells being Miro1/2) and their motor adaptors Trak1 and Trak2 (Stowers et al., 2002; Glater et al., 2006; Russo et al., 2009).

Another dynamic aspect of mitochondria is the selective removal of dysfunctional mitochondria via autophagy, a quality-control mechanism named mitophagy that ensures a healthy mitochondrial population. Mitophagy in conjunction with mitochondrial biogenesis regulates the mitochondrial content that is needed under various conditions to meet cellular metabolic demand. Several key factors and pathways have been identified in regulating mitophagy and the most well-known pathways include the Pink1/Parkin pathway and Nix pathway. Mitophagy consista in the sequestration of the defective organelle into a double membrane phagosome that is later fused with lysosomes forming autolysosomes, where the contents are degraded and can be recycled.

The turnover rate varies depending on different species, cell types, and physiological conditions within single cells. It has been estimated that the turnover of mitochondria in both young and aged rat liver, heart, and brain is 9.3, 17.5, and 24.4 days, respectively (Menzies and Gold, 1971).

\section{Mitochondrial Fission}

Mitochondrial fission is a multi-step process where the recruitment of the large GTPase Drp1 plays a crucial role. Drp1 is evolutionary conserved across Caenorhabditis elegans (Labrousse et al., 1999), yeast (Bleazard et al., 1999), and mammals (Smirnova et al., 2001). It is mainly a cytosolic protein, but is dynamically recruited to mitochondrial and peroxisomal 
membranes, where it oligomerizes and drives membrane constriction in a GTP-dependent manner (Tilokani et al., 2018). Genetic ablation of Drp1 leads to a drastic elongation of both mitochondria and peroxisomes (Koch et al., 2003) in multiple cell lines and a variety of animal models (Ishihara et al., 2009).

During mitochondrial fission, Drp1 is recruited from the cytosol to the mitochondrial outer membrane, where it forms a ring-like structure around the mitochondria leading to the narrowing of the membrane (Smirnova et al., 2001; Fröhlich et al., 2013). Then, GTP hydrolysis enhances this membrane constriction (Mears et al., 2011) which marks a potential future site of mitochondrial scission.

As Drp1 lacks a Pleckstrin Homology (PH) domain to bind membrane phospholipids directly, additional adaptor proteins are needed to help the recruitment of Drp1 onto the outer membrane (Tilokani et al., 2018). In mammalian cells, three adaptor proteins have been identified serving as the receptor proteins of Drp1--the tail-anchored proteins mitochondrial fission factor (MFF) (Gandre-Babbe and van der Bliek, 2008) and mitochondrial dynamics proteins 49 and 51 (MiD49 and MiD51) (Palmer et al., 2011; Losón et al., 2013). Although both MFF and MiDs serve as the receptor protein of Drp1, their roles differ slightly in regulating Drp1 mediated fission, which leads to different mitochondrial morphology outcomes in cell lines with either MFF or MiDs knocked out. Overexpression of MFF leads to a fragmented network whereas MFF knockout induces mitochondrial and peroxisome elongation (Otera et al., 2010), accompanied by a decrease in Drp1 mitochondrial recruitment. However, MiD49 and MiD51 mutants show completely opposite phenotypes. Overexpression of MiDs leads to mitochondrial elongation due to Drp1 sequestration (Palmer et al., 2011; Zhao et al., 2011). Either Mid49 or MiD51 can mediate Drp1 recruitment and mitochondrial fission in the absence of Mff, 
suggesting that multiple receptors can recruit Drp1 to mitochondria for fission independent of each other (Losón et al., 2013).

In addition to Drp1 regulated mitochondrial membrane constriction step, it has been found that ER is required for the initiation of the mitochondrial division (Friedman et al., 2011). The pre-constriction step at the ER-mitochondria contact site is thought to mark the future fission site and decrease the average mitochondrial diameter from approximately $300-500 \mathrm{~nm}$ to $\sim 150$ nm, further allowing Drp1-oligomeric ring formation (Friedman et al., 2011). Drp1 and its adaptor proteins are subsequently recruited to these mitochondria-ER contact sites prior to mitochondrial division (Friedman et al., 2011). Furthermore, recent studies suggest that the canonical GTPase Dnm2, which was known to regulate endocytic vesicle fission, was involved in catalyzing the final step of membrane scission (Lee et al., 2016) to facilitate the complete separation of mitochondria. However, another study suggests that Dnm2 is not involved in fission after all, and it is DRP1 that is crucial to the final fission step (Fonseca et al., 2019). Debates remain in the field as to determine the actual role of Dnm2 in mitochondrial fission process, if any.

\section{Mitochondrial Fusion}

Mitochondrial fusion is the merger of two individual mitochondria into a new mitochondrion. One of the most important and well known functions of fusion is to regulate mitochondrial morphology. Each cell type has a characteristic profile of mitochondrial morphology that is maintained by the balanced control of mitochondrial fusion and fission. For instance, interphase fibroblast cells in culture typically have tubular mitochondria, ranging from 
short tubules to long tubules to interconnected tubular networks. When fusion is blocked, the tubules fragment rapidly into small spheres as a result of unopposed fission (Chen et al., 2003a). When fission is blocked, the tubules elongate dramatically and show more interconnections due to unopposed fusion. Imbalance between fusion and fission is related with the onset and progression of several familial and sporadic neurodegenerative disorders (Knott and BossyWetzel, 2008). Despite the importance of fusion and fission balance control, little is known about how cells maintain a particular balance between fusion and fission events. In one study, researchers have shown that fission events often occur shortly after fusion events, suggesting that there may be an underlying feedback mechanism linking the two processes (Twig et al., 2008).

Two major categories of fusion have been studied so far: the full complete fusion and the 'kiss and run' type of fusion. In a typical mitochondrial fusion reaction, two mitochondria come into contact end-to-end (longitudinal interaction) or side-by-side (oblique interaction), and the membrane fusion event occurs at the contact site. In addition to two or more mitochondria fusing into one, fusion can also refer to single mitochondrion fusing from one end to the other, forming the ring-like donut shape structure. Since mitochondria are double-membraned organelles, one complete fusion process consists of an outer membrane fusion followed by an inner membrane fusion. These two membrane fusion events occur close in time, but are spatially and temporally separable (Song et al., 2009). The end result of membrane fusion is content mixing, with the matrix components diffusing throughout the new mitochondrion.

Besides the obviously complete and relatively prolonged fusion events, some fusion events called 'kiss-and-run' fusion, are extremely transient, and the original mitochondria quickly separate after content exchange, with no obvious structural rearrangement (Liu et al., 
2009). It has been found that mitochondria moving along the same microtubule usually undergo the complete fusion whereas mitochondria traveling along distinct but spatially close microtubules go through the transient ('kiss-and-run') fusions. Cultured rat cardiomyocytes expressing photoactivatable fluorescent proteins targeted to the mitochondrial matrix together with another mitochondrial fluorescent protein were used to observe such events. When individual mitochondria were laser photo-activated, the fluorescence marked mitochondria exchange matrix contents with other mitochondria during transient encounters as shown by mixing of converted fluorescence. However, since the fusion events are so transient, only content mixing were observed without obvious morphology change on either mitochondrion (Liu et al., 2009). A basic difference between transient fusion and complete fusion lies in the extent of the mixing of the mitochondrial components. The restricted mixing of integral membrane components during transient fusion may be of relevance for preservation of the original morphology of the merging organelles (Liu et al., 2009). The observation of this flexible transient fusion type implies that fusion pores can rapidly open and close between two mitochondria, further suggesting that besides regulating mitochondrial morphology, content exchange is also an important function of mitochondrial fusion (Chan, 2020).

Although mtDNA is located in the matrix and content exchange happens during fusion events, mixing of mtDNA genomes during fusion were not obviously observed. A previous study showed that when two different cell lines harboring distinct non-overlapping mtDNA deletions fuse together, the two sets of mtDNA functionally complemented each other and together restored the normal level of protein synthesis, however, the two sets of mtDNAs remained in discrete nucleoids without obvious intermixing. The result suggests that during mitochondrial 
fusion, the nucleoids regulate and maintain their genetic content instead of freely exchange mtDNA components among each other (Gilkerson et al., 2008).

In mammalian cells, three large GTPases, which bind to GTP and hydrolyze GTP to GDP to facilitate various biochemical processes, are discovered to be essential for mitochondrial fusion. The mitofusins Mfn1 and Mfn2 are transmembrane GTPases embedded in the mitochondrial outer membrane. They face the cytosol and are responsible for mediating mitochondrial outer membrane fusion (Rojo et al., 2002; Santel and Fuller, 2001). OPA1 has multiple isoforms, some anchored on the mitochondrial inner membrane and others lacking a transmembrane anchor (Delettre et al., 2000a). It mediates inner membrane fusion. Depletion of any of these three GTPases results in severely reduced levels of mitochondrial fusion (Chen et al., 2005b; Cipolat et al., 2004; Griparic et al., 2007a; Song et al., 2007a).

\section{Molecular Machinery of Mitochondrial Fusion}

\section{Outer Membrane Fusion (MFN1\&2)}

The mitofusins were the first proteins found to be important for mitochondrial fusion. The first mitofusin gene, fuzzy onions ( $f z o$ ), was discovered in Drosophila, the deletion of which leads to male infertility (Hales and Fuller, 1997). The gene also has homologs in mammals, nematodes and yeast, and later studies have shown that the fusion functions are conserved across these species. In early post-meiotic spermatids, all mitochondria aggregate beside each haploid nucleus, then fuse into two giant mitochondria that wrap around each other to form a spherical structure named the Nebenkern. When viewed in cross section by transmission electron microscopy (TEM), the Nebenkern resembles an onion slice, and therefore early round spermatids are also known as the 'onion stage'. In fzo mutants, the Nebenkern structure 
formation was disrupted, and in TEM images, instead of having round onion slices, they are irregular and 'fluffy' and 'fuzzy', giving the gene the name 'fuzzy onions'. Imaging studies showed that mutation of Fzo resulted in failure of the mitochondria to fuse. It appears that Fzo function is restricted to spermatids during a brief developmental stage in spermatogenesis. A homologous gene, Marf/Dmfn, has been found to be expressed more widely and function more broadly to mediate mitochondrial fusion in other tissues (Hwa et al., 2002).

Mammalian cells have two mitofusins, Mfn1 and Mfn2, that are anchored on the mitochondrial outer membrane. Deletion of either Mfn11 or Mfn2 leads to fragmented mitochondria, due to unopposed Drp1-mediated fission. Conversely, overexpression of Mfn1 or Mfn2 leads to highly tubulated mitochondria, an indication of elevated fusion (Rojo et al., 2002; Santel and Fuller, 2001). Knockout mouse models further demonstrate the essential roles of these proteins for mitochondrial fusion as well as other vital functions (Chen et al., 2003a, 2005b). Homozygous Mfn1 and Mfn2 knockouts are embryonically lethal, suggesting that Mfn1 and Mfn2 are both required for embryonic development, and may have a cooperative relationship in the early stage of embryogenesis. Mouse embryonic fibroblasts (MEFs) lacking either Mfn1 or Mfn2 have highly fragmented mitochondria compared to the tubular network observed in wildtype cells. Fusion assays indeed indicate a great reduction in the levels of mitochondrial fusion in single knockout MEFs (Chen et al., 2003a) and a complete loss of fusion in cells lacking both Mfn1 and Mfn2 (Chen et al., 2005b).

When mitochondrial fusion rates are reduced, the mitochondrial population fragments into short tubules or small spheres because of ongoing mitochondrial fission in the face of less fusion. These observations support the idea that mitochondrial morphology is dictated by a 
balance between fusion and fission, and although there are some interplay between these two

processes. Although both have been shown to be required for mediating mitochondrial fusion, it has also been reported that Mfn1 and Mfn2 have distinct functions. In mouse models, only Mfn2 mutant mice show placental defects and Mfn1 mutant embryos display completely normal placental development. Mfn1 tethers mitochondrial membranes, depending on GTP hydrolysis, with a higher efficiency than Mfn2, which corresponds to changes in the oligomeric states of the Mfn-containing complex. These properties reflect differences in the GTPase activity of the Mfn proteins; purified Mfn1 exhibited eightfold higher activity than Mfn2 (Ishihara et al., 2004). In addition to mitochondrial fusion, Mfn2 has been implicated in maintaining the sites of contact between the mitochondria and the endoplasmic reticulum (de Brito and Scorrano, 2008).

\section{Inner Membrane Fusion (OPA1)}

Inner membrane fusion is mediated by Optic Atrophy 1 (Opa1), a large GTPase of the dynamin superfamily. Going from $\mathrm{N}$-terminus towards the $\mathrm{C}$-terminus, the protein has a mitochondrial targeting sequence, a transmembrane domain, a coiled coil domain and other functional domains (GTPase domain, middle domain, and GTPase effector domain). Opa1 was first identified as the gene mutated in autosomal dominant optic atrophy, a human disease showing progressive loss of visual acuity, and bilateral temporal atrophy of the optic nerve with an early onset within the first two decades of life (Alexander et al., 2000b).

Cells lacking Opa1 show mitochondrial outer membrane fusion, but the fusion process cannot proceed further into sequential inner membrane fusion, resulting in fragmented 
mitochondria in Opa1-null cells (Song et al, 2007). Unlike mitofusins, Opa1 is needed on only one of two opposing mitochondria for inner membrane fusion to occur (Song et al., 2007a, 2009; Ban et al., 2017a).

Opa1 is post-translationally regulated by proteases

In human cells, eight RNA splice forms of Opa1 are generated from alternative splicing of exons 4, 4b, and 5b (Fig 1.2). All RNA splice forms contain an N-terminal mitochondrial targeting sequence (MTS). The MTS is removed by the matrix processing protease (MPP) during import into the mitochondrial matrix, leaving a very short fragment remaining in the matrix and the transmembrane segment anchoring OPA1 on the inner membrane, whereas the majority of OPA1 is in the intermembrane space. At least two proteolytic cleavage sites are present in the intermembrane space segment of Opa1, enabling membrane-anchored long form of OPA1 (1OPA1) to be further cleaved by proteases, leading to the generation of short forms of OPA1 (sOPA1) lacking the N-terminal transmembrane domain. The universal exon 5, which is present in all isoforms, encodes a proteolytic processing site S1. When S1 site gets recognized and cleaved by OMA1, a mitochondrial inner membrane metalloprotease, partial OPA1 long forms get processed into short forms, which do not have a membrane anchor and only exist in the intermembrane space. The short forms are soluble in principle, but known to form complexes with the membrane-bound long Opa1 isoforms to modulate fusion activity. Additional proteolytic cleavage site, $S 2$, is found in half of the splice forms $(4,6,7$, and 8$)$, which is encoded by the exon 5b and cleaved by YME1L, an m-AAA protease (Song et al., 2007a). The 
OMA1 and YME1L proteases together regulate OPA1 processing (Song et al., 2007a; Anand et al., 2014a).

The role of Opa1 isoforms in mediating fusion

An intriguing question is why this diversity of proteolytic processing of OPA1 exists. It has been shown recently that both the long and short OPA1 isoforms can independently restore mtDNA content, maintain respiratory function, stabilize OXPHOS complex, and maintain energetic activity (Lee et al., 2017)(Del Dotto et al., 2017b). However, there are controversial opinions on their significance to fusion activity. It is clear that, under basal conditions, a combination of both long and short isoforms is required for physiological levels of mitochondrial fusion (Song et al., 2007a). For example, when expressing either long or only short OPAlin Opa1-null cells, little mitochondrial fusion is detected. When both isoforms are co-expressed, partial fusion activity is restored in the cells (Song et al., 2007a). Under pathological conditions, such as dissipation of mitochondrial inner membrane potential, Oma1 is activated and promotes complete processing of OPA1 to short isoforms (Griparic et al., 2007a; Mishra et al., 2014a). This complete shift to short isoforms results in inactivation of fusion activity. It has also been proposed that long and short Opa1 serve completely opposite roles in mitochondrial dynamicslong OPA1 alone is sufficient for mediating fusion whereas short Opa1 promotes fission. SOPA1 accumulation has been linked to mitochondrial fragmentation in Yme1L null MEFs, where an increased level of short Opa1 has been observed due to OMA1 activation induced increased cleavage. Also, when expressing s-OPA1 in Oma1/Yme1L DKO MEFs, the mitochondria network became more fragmented compared to control. However, it should be 
noted that several studies have shown that increased levels of overall OPAl proteins, regardless of 1 - or s-OPA1, can cause mitochondrial fragmentation, therefore this could be an artifact induced by total OPAl protein levels instead of s-Opa1.

Several lines of evidence indicate that long OPA1 by itself is capable of mediating mitochondrial fusion under certain conditions. In Opa1-null cells expressing only long OPA1 isoforms, there is extensive elongation of mitochondria if the cells are treated with cycloheximide, a protein translation inhibitor which induces a condition called stress-induced hyperfusion (Tondera et al., 2009a). Additionally, cells with both the Oma1 and Yme1L proteases knocked out, where OPA1 cannot be processed into the short forms, contain some tubular mitochondria and mitochondrial fusion activity (Anand et al., 2014a). Lastly, in a recent study, the authors developed an in vitro assay where recombinant long Opa1 isoforms incorporated into liposome membranes showed fusion activity against other liposomes, as long as the opposing liposomes contained cardiolipin, a vital lipid component on the inner membrane and known to be a binding partner of OPA1 (Ban et al., 2017a). However, the study also showed evidence that short OPA1 can modulate fusion where adding s-OPA1 into 1-OPA1 plus liposome system can enhance fusion activity (Ban et al., 2017a). Even more, short OPA1 alone mediates GTP-dependent fusion when added to liposomes containing cardiolipin (Ban et al., 2018b).

Taken together, these results indicate that both the long and short forms of Opa1 can display inherent fusion activity. In cells under basal conditions, long or short OPA1 alone shows little or no fusion activity (Song et al., 2007a). Under stress conditions, especially conditions associated with stress-induced hyperfusion (Tondera et al., 2009a), the fusion activity of the long isoform is activated by unknown mechanisms, which could potentially be explained by 
temporary structural change or enhanced activity binding to cardiolipin. Short Opa1 can substantially regulate the fusion activity of long Opa1 (Ban et al., 2017a, 2018b), and in fact, cleavage of long Opa1 into the short isoform activates its membrane fusion activity (Mishra et al., 2014a).

\section{The role of Opal in cristae maintenance and energetics}

In addition to its inner membrane fusion function, depletion of OPA1 protein in cells leads to increased apoptotic sensitivity and decreased mtDNA content and stability, as well as reduced energetic activity. These are correlated with the change in cristae structure, including cristae number, shape, and cristae junction tightness. Oligomerization of OPA1 appears to be a mechanism that regulates apoptosis by maintaining the tightness of cristae junctions (Frezza et al., 2006).

The apoptosis regulation function of OPA1 was discovered in a study where the authors suggest that under normal conditions, OPA1 protein oligomerized to keep the cristae junction tight, whereas during tBid-induced apoptosis, OPA1 complexes got disrupted and the cristae junctions widened, allowing for the release of cytochrome $\mathrm{c}$ from the intra-cristal space (Frezza et al., 2006). This cristae junction widening was not seen in other studies, and in fact some studies showed contrary phenotypes in which during apoptosis, the cristae junctions became tighter and narrower (Yamaguchi et al., 2008; Sun et al., 2007). Further research is needed to confirm this cristae junction change phenotype and the role of Opa1 in regulating this phenotype under stress induced apoptosis. Despite contradictory results on the cristae junction morphology, past studies showed consistently that OPA1 complex disassembly is necessary for cytochrome c release during apoptosis (Frezza et al., 2006; Yamaguchi et al., 2008). Therefore, it is more 
likely that the assembled OPA1 complex instead of cristae junction wideness/morphology is the key regulator of apoptosis induced cytochrome $c$ release, pointing to a functional link between OPA1 and cytochrome $c$.

Through the immuno-EM images of OPA1 within mitochondria, it is obvious that OPA1 is enriched along the central neck area of the cristae with gradual decreases toward cristae junction and tip (Barrera et al., 2016). The most striking ultrastructural defect in OPA1 deficiency is the disruption of cristae structure, showing greatly decreased cristae number and the loss of cristae tightness (Olichon et al., 2003; Griparic et al., 2007a; Lee et al., 2017a). OPA1 deficiency causes defects in respiration and the respiratory complex, likely due to the loss membrane in which respiratory complexes and super-complexes assemble (Lee et al., 2017a; Del Dotto et al., 2017b). It is likely that the functions of OPA1 in energetic maintenance and apoptotic resistance are attributed to the structural and maintaining cristae tightness (Cogliati et al., 2013).

Two recent studies have demonstrated the important role of s-OPA1 in energetic maintenance (Lee et al., 2017a; Del Dotto et al., 2017b). Different isoforms were put back into the Opa1 null cells and studied individually. In contrast to the prevalent notion that s-OPA1 is just the nonfunctional proteolytic product, it was found that s-OPA1 by itself is sufficient to maintain respiratory function independent of 1-OPA1 (Lee et al., 2017a; Del Dotto et al., 2017b). The expression of a single form of S1-cleaved s-OPA1 in OPA1-KO cells was able to maintain mtDNA stability, respiratory complexes, and cristae structure, indistinguishable from that of LOPA1 (Lee et al., 2017a). No functional distinction among the eight variants were found in restoring energetic activity (Del Dotto et al., 2017b). Importantly, the ability of s-Opa1 alone for maintaining energetic function without being able to directly mediate fusion supports the 
previous idea that the fusion function and cristae maintenance and energetics function of OPA1 are mechanistically separated (Frezza et al., 2006). The energetic phenotypes of OPA1 deficiency include respiration defects, decreased levels of respiratory complexes and mtDNA content, all of which are likely due to the lack of adequate cristae structure maintenance in the absence of OPA1. Cristae membranes are the assembly platform for respiratory complexes, and therefore, when cristae structures change or cristae numbers decrease, respiratory complexes become unstable and degraded, which later could deteriorate into a respiration defect.

Opa1 knockout cells show an almost complete loss of cristae structure, suggesting the importance of Opa1 in cristae maintenance. It was shown in one study that the expression of either 1- or s-OPA1 increased the cristae number, indicating that either isoform is able to maintain cristae structure independent of fusion activity. Moreover, OPA1 mutants with defective GTPase domain can rescue the number of cristae, but with an abnormal morphology. The cristae in Opa1 null cells infected with Opa1 GTPase mutants were swollen and round (balloon shape), indicating a loss of cristae tightness (Lee et al., 2017a).

In Opa1 mutant cells and mice, mtDNA levels are dramatically decreased (Elachouri et al., 2011; Chen et al., 2010). The role of Opa1 in mtDNA maintenance is thought to be linked to the fusion activity (Chen et al., 2010; Patten et al., 2014). However, since mtDNA is attached to the IM, the mtDNA decrease with OPA1 deficiency may also be due to the cristae and inner membrane disruption. The two studies on s-Opa1 function show that short Opa1 can also rescue mtDNA level, regardless of the minimal ability for fusion (Lee et al., 2017a; Del Dotto et al., 2017b). The new finding that s-OPA1 alone, without 1-OPA1, is able to support the formation of cristae, as well as the maintenance of cristae tightness, is unexpected but significant, as it 
prompts the revision of the current paradigm of 1- and s-OPA1 functions.

\section{Physiological roles of mitochondrial fusion}

We will be discussing some key functions of mitochondrial fusion and fission in the next section. Fusion and fission fulfill important physiological functions for the mitochondria. Knockout mouse models of any fusion genes result in embryonic lethality, further indicating the vital roles of fusion process in maintaining mitochondrial and cellular functions. Next, we will briefly discuss the known roles of fusion and how they are physiologically linked with various cellular processes. The functions of mitochondrial dynamics are still not fully understood and researchers are actively looking for additional aspects that fusion and fission have in regulating mitochondrial and cellular functions. Here, we mainly talk about five well studied functions on the list--

\section{Mitochondrial Morphology Regulation}

The balance of fusion and fission appears to be essential for controlling the size, shape, and abundance of mitochondria within a given cell. When the balance shifts towards more fusion, the mitochondria exhibit a larger and longer shape, whereas shifting towards the opposite direction results in smaller and more punctated mitochondria. An intriguing but still unsolved question arises as how mitochondrial morphology may affect the function of mitochondria. It has been suggested that mitochondrial shape may directly affect their bioenergetic activity. Elongated mitochondria are thought to have enhanced ATP production efficiency and can protect mitochondria from stressful conditions, whereas fragmented mitochondria are usually seen under 
pathological conditions such as uncoupling and membrane depolarization. Not enough experimental results have proven the causal relationship between mitochondrial fragmentation and low energy production. A recently published study suggests that the short form of OPA1, which is usually linked with mitochondrial fragmentation, is more capable of ATP generation compared to the long form only (Lee et al., 2020a).

Evidence from neurons suggests that certain cell types may be more sensitive for mitochondrial shape change than others. In neurons, mitochondria need to be transported out from the cell body, traveling along axons and reaching to the dendritic terminals. Smaller mitochondria are thought to be transported more efficiently than larger and longer mitochondria during this process. Overexpression of Drp1 lead to increased mitochondrial fission, and has been shown to increase dendritic mitochondria, associated with enhanced density of synapses ( $\mathrm{Li}$ et al., 2004; Verstreken et al., 2005). The failure of elongated mitochondria to be transported into nerve terminals may result from entanglement of long mitochondria in the soma. Blocking fission process causes mitochondria elongation and interconnection, decreasing the efficiency of mitochondrial transport into axons, which can potentially be explained by mitochondrial entanglement in the cell body. Fusion process disruption does not increase the transport efficiency, although mitochondria can get fragmented under this condition. This can be explained by the direct physical interaction between fusion protein mitofusins and transport proteins including Miro and Milton (Misko et al., 2010). By disrupting the mitochondrial outer membrane fusion, the transport machinery is severed and cannot support axonal mitochondrial transport.

\section{Inter-mitochondria Content Exchange Facilitation}


Mitochondrial fusion involves not only membrane mixing but more importantly matrix mixing and content exchange. The content exchange feature has extensive benefits to the cell, including maintaining a homogeneous mitochondrial population and tolerance of mtDNA mutations. Since mitochondria import most of the proteins from cytosols, the distribution and components of different proteins among various mitochondria within a cell may differ slightly due to spatial and temporal difference. Without fusion, the variations get more enhanced and eventually lead to inter-mitochondrial differences, which can lead to pathogenesis if a certain portion of mitochondria gets unhealthy. Similarly, fusion enables ameliorating pathological effects caused by excessively mutated and damaged mtDNA. Most mtDNA mutations causing mitochondrial disease are recessive, and need to reach a high percentage of $60-90 \%$ to be pathogenic. By fusing a mitochondrion with no or low level of mtDNA with a mitochondrion with relatively high level of mtDNA, the heteroplasmy level can be mitigated and therefore can avoid respiratory defects caused by high level mtDNA mutations (Chen et al., 2010).

\section{mtDNA Stability Maintenance}

Cells with no mitochondrial fusion contain extremely low levels of mtDNA. MEFs lacking both mitofusins or OPA1 alone retain only 1/3 of the mtDNA amount compared to that in WT cells (Chen et al., 2010). It is well established that mtDNA encodes 13 polypeptides that are part of the OXPHOS complexes, therefore, reduced levels of mtDNA lead to a huge reduction of OXPHOS activity. The OXPHOS deficiency is likely a combination of mtDNA loss and fusion defect, especially in OPA1 null cells, as OPA1 has been shown to be involved in cristae structure maintenance and respiratory complex stability. On the other hand, cells deficient 
in mitochondrial fission also exhibit mtDNA defects. In fission deficient cells, mtDNA are not properly distributed, rather the mtDNA nucleoids aggregate and form large structures that deform mitochondrial tubules (Ban-Ishihara et al., 2013). The clustering of nucleoids results in uneven distribution of mtDNA within the mitochondria and causes mosaic OXPHOS deficits in cells (Ishihara et al., 2015). This dependence of the mtDNA level on fusion and fission activity is not only seen in cultured cells but also in various high energy demanding tissues such as mouse skeletal muscle (Chen et al., 2010) and cardiomyocytes (Ishihara et al., 2015).

\section{Cellular Stress Adaptation}

Previous studies have shown that mitochondrial fusion and fission activity can be modulated according to various cellar stresses. Certain stressors such as RNA transcription inhibitors, protein translation inhibitors, UV irradiation, and nutrient starvation inducers all cause super elongated mitochondria (Tondera et al., 2009a). This phenomena is termed stress-inducedmitochondrial-hyperfusion (SIMH). Fusion proteins MFN1 and OPA1 are necessary for this elevated fusion process in response to the first three stress inducers. Along with the hyperfusion process, cells showed increased ATP production to better survive under these stresses. Fusion mutant cells show increased vulnerability to stress and more readily undergo apoptosis, possibly due to the inability of initiating the hyperfusion process.

Other studies have indicated the role of mitochondrial fission in mitochondrial elongation under serum and amino acid starvation (Gomes et al., 2011; Rambold et al., 2011). In contrast to conditions discussed above, inhibition of fission instead of activation of fusion results in mitochondrial elongation. More specifically, Drp1 recruitment to mitochondria is disrupted when 
cells are starved. The fission inhibition is thought to be a mechanism for mitochondria to escape autophagy/clearance under this particular stress condition. Cells that possess mutations inhibiting the elongation process show an elevated level of mitophagy under the starvation condition.

\section{Mitochondrial Quality Control}

The degradation of mitochondria is completed by mitophagy, where damaged mitochondria are degraded through a specialized autophagy machinery starting with engulfment by autophagosome followed by fusing with lysosome forming auto-lysosome and then later for final degradation in lysosome. During the first step of mitophagy, the damaged mitochondria need to be recognized and isolated from the healthy mitochondria, and this process is completed by the mitochondrial fusion and fission processes. To begin with, the dysfunctional mitochondria, which usually show inner membrane depolarization, can no longer fuse with the rest of the healthy population due to increased processing of Opa1 induced by the activation of Oma1 (Twig et al., 2008). Additionally, OXPHOS activity also regulates Opa1 cleavage and function, with increased fusion under highly oxidative conditions. Cells that do not have proper OXPHOS activity also cannot fuse due to Opa1 inactivity. On the other hand, fission can promote the segregation of damaged mitochondria as well as further divide targeted mitochondria into smaller pieces to facilitate the autophagosome engulfment process. In mammalian cells, inhibition of Drp1-mediated mitochondrial fission prevents Parkin-induced mitophagy, a well-known pathway regulating selective mitochondrial degradation. Finally, during Parkin-mediated mitophagy, unbiquitination of mitofusins and other mitochondrial outer membrane proteins are observed and lead to protein degradation (Tanaka et al., 2010). This removal of fusion machinery further prevents re-fusion of any targeted mitochondria back into the system. 


\section{Outline of thesis}

In my thesis, I will be discussing the novel finding of the inner membrane fusion protein OPA1 on its cleavage process and related physiological functions. One of the most important issues regarding the long and short forms of OPA1 remains unelucidated-how both forms coordinate together for mediating fusion, in particular, how important is short OPA1 in the fusion process, given the fact that half of the isoforms produce exclusively short forms. What conditions favor more balanced long and short forms, more proteolytic cleaved forms, whereas other conditions may prefer exclusively long forms. To better understand the role of s-OPA1, we used CRISPR-Cas9 gene editing to generate Opa1 cleavage site knockout cell lines and mouse models. In Chapter 2, I will talk about how a novel S3 cleavage site of Opa1 was discovered, the protease cleaving at the site, the exact cleavage site within the exon, and the physiological function of this novel cleavage site as well as how it is involved in mediating fusion activity. Lastly, in Chapter3, I will briefly discuss the implications of my findings, how it can help improve the current model together with other studies, and the future research needed to further address the remaining unknown issues. 


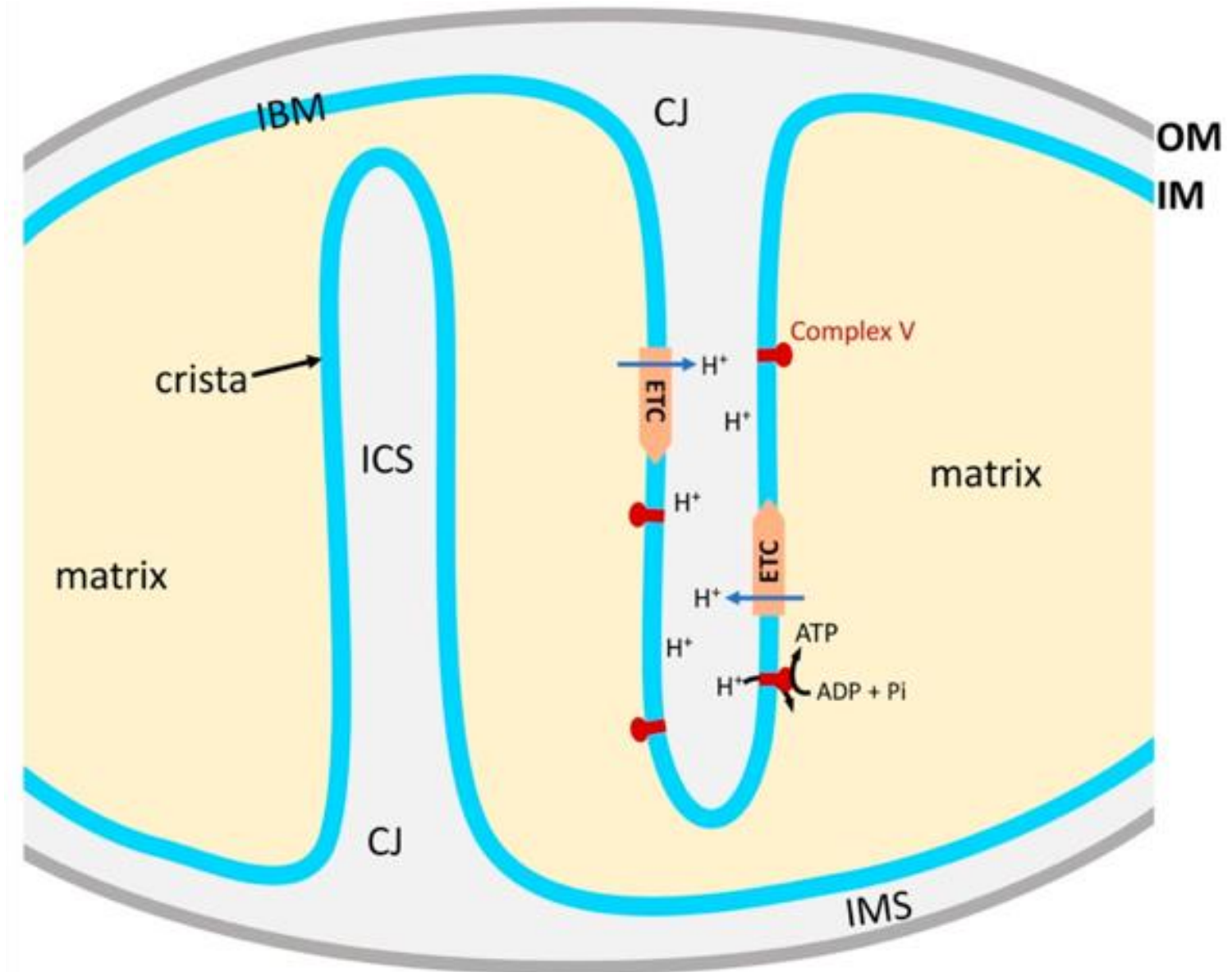

Fig. 1.1 Mitochondria Cristae Structure (modified from Lee et al., 2020a). OM: outer membrane; IM: inner membrane; IBM: inner boundary membrane; IMS: inter membrane space; CJ: cristae junction; ICS: intra cristal space; ETC: electron transport chain. 

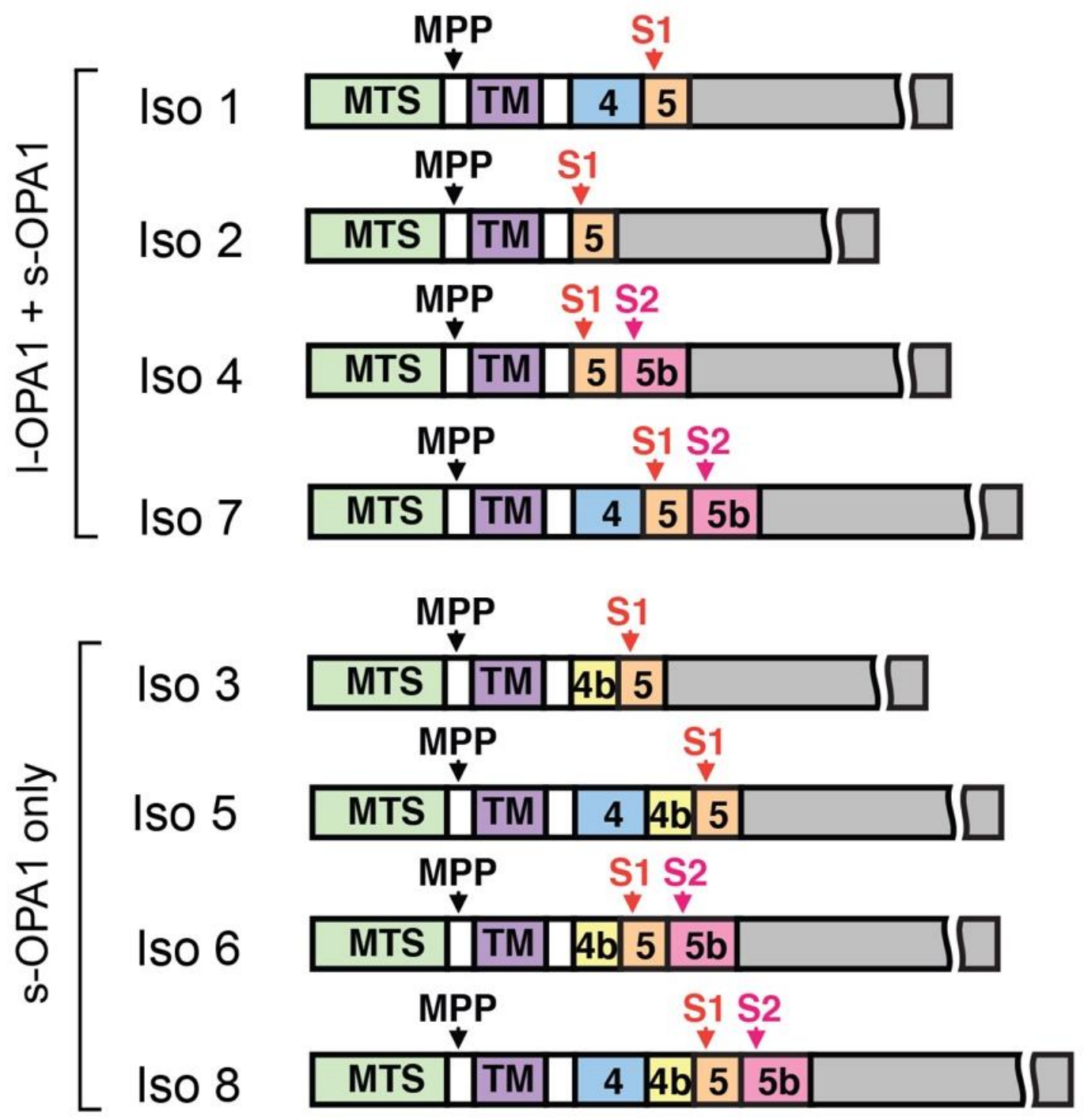

Fig. 1.2 Human OPA1 isoforms overview. At least 8 Opal mRNA splice variants exist in human cells. Isoforms 1, 2, 4, and 7 generate both 1- and s- OPA1, whereas isoforms 3, 5, 6, and 8 produce only s-OPA1 due to the presence of exon4b. MPP: mitochondrial processing peptidase; S1: cleavage site 1 , locates within exon 5; S2: cleavage site 2, locates within exon 5 b. 


\section{References}

Akepati, V.R., E.-C. Müller, A. Otto, H.M. Strauss, M. Portwich, and C. Alexander. 2008. Characterization of OPA1 isoforms isolated from mouse tissues. J. of Neurochemistry. 106:372-383. doi:10.1111/j.1471-4159.2008.05401.x.

Alavi, M.V., S. Bette, S. Schimpf, F. Schuettauf, U. Schraermeyer, H.F. Wehrl, L. Ruttiger, S.C. Beck, F. Tonagel, B.J. Pichler, M. Knipper, T. Peters, J. Laufs, and B. Wissinger. 2007. A splice site mutation in the murine Opal gene features pathology of autosomal dominant optic atrophy. Brain. 130:1029-1042. doi:10.1093/brain/awm005.

Alexander, C., M. Votruba, U.E. Pesch, D.L. Thiselton, S. Mayer, A. Moore, M. Rodriguez, U. Kellner, B. Leo-Kottler, G. Auburger, S.S. Bhattacharya, and B. Wissinger. 2000a. OPA1, encoding a dynamin-related GTPase, is mutated in autosomal dominant optic atrophy linked to chromosome 3q28. Nat. Genet. 26:211-215. doi:10.1038/79944.

Anand, R., T. Wai, M.J. Baker, N. Kladt, A.C. Schauss, E. Rugarli, and T. Langer. 2014a. The iAAA protease YME1L and OMA1 cleave OPA1 to balance mitochondrial fusion and fission. J. Cell Biol. 204:919-929. doi:10.1083/jcb.201308006.

Ban, T., T. Ishihara, H. Kohno, S. Saita, A. Ichimura, K. Maenaka, T. Oka, K. Mihara, and N. Ishihara. 2017a. Molecular basis of selective mitochondrial fusion by heterotypic action between OPA1 and cardiolipin. Nat. Cell Biol. 19:856-863. doi:10.1038/ncb3560.

Ban, T., H. Kohno, T. Ishihara, and N. Ishihara. 2018a. Relationship between OPA1 and cardiolipin in mitochondrial inner-membrane fusion. Biochim. Biophys. Acta. Bioenerg. 1859:951-957. doi:10.1016/j.bbabio.2018.05.016.

Ban-Ishihara, R., T. Ishihara, N. Sasaki, K. Mihara, and N. Ishihara. 2013. Dynamics of nucleoid structure regulated by mitochondrial fission contributes to cristae reformation and release of cytochrome c. Proc. Natl. Acad. Sci. U.S.A. 110:11863-11868. doi:10.1073/pnas.1301951110.

Barrera, M., S. Koob, D. Dikov, F. Vogel, and A.S. Reichert. 2016. OPA1 functionally interacts with MIC60 but is dispensable for crista junction formation. FEBS Lett. 590:3309-3322. doi:10.1002/1873-3468.12384.

Bayrhuber, M., T. Meins, M. Habeck, S. Becker, K. Giller, S. Villinger, C. Vonrhein, C. Griesinger, M. Zweckstetter, and K. Zeth. 2008. Structure of the human voltagedependent anion channel. Proc. Natl. Acad. Sci. U.S.A. 105:15370-15375. doi:10.1073/pnas.0808115105. 
Bleazard, W., J.M. McCaffery, E.J. King, S. Bale, A. Mozdy, Q. Tieu, J. Nunnari, and J.M. Shaw. 1999. The dynamin-related GTPase Dnm1 regulates mitochondrial fission in yeast. Nat. Cell Biol.. 1:298-304. doi:10.1038/13014.

de Brito, O.M., and L. Scorrano. 2008. Mitofusin 2 tethers endoplasmic reticulum to mitochondria. Nature. 456:605-610. doi:10.1038/nature07534.

Carelli, V., and D.C. Chan. 2014. Mitochondrial DNA: Impacting central and peripheral nervous systems. Neuron. 84:1126-1142. doi:10.1016/j.neuron.2014.11.022.

Chan, D.C. 2019. Mitochondrial dynamics and its involvement in disease. Annu. Rev. Pathol. doi:10.1146/annurev-pathmechdis-012419-032711.

Chen, H., A. Chomyn, and D.C. Chan. 2005a. Disruption of fusion results in mitochondrial heterogeneity and dysfunction. J. Biol Chem. 280:26185-26192.

Chen, H., S.A. Detmer, A.J. Ewald, E.E. Griffin, S.E. Fraser, and D.C. Chan. 2003a. Mitofusins Mfn1 and Mfn2 coordinately regulate mitochondrial fusion and are essential for embryonic development. J. Cell Biol. 160:189-200. doi:10.1083/jcb.200211046.

Chen, H., J.M. McCaffery, and D.C. Chan. 2007. Mitochondrial fusion protects against neurodegeneration in the cerebellum. Cell. 130:548-62. doi:10.1016/j.cell.2007.06.026.

Chen, H., M. Vermulst, Y.E. Wang, A. Chomyn, T.A. Prolla, J.M. McCaffery, and D.C. Chan. 2010. Mitochondrial fusion is required for mtDNA stability in skeletal muscle and tolerance of mtDNA mutations. Cell. 141:280-289. doi:10.1016/j.cell.2010.02.026.

Cipolat, S., O. Martins de Brito, B. Dal Zilio, and L. Scorrano. 2004. OPA1 requires mitofusin 1 to promote mitochondrial fusion. Proc. Natl. Acad. Sci. U.S.A. 101:15927-15932. doi:10.1073/pnas.0407043101.

Cogliati, S., C. Frezza, M.E. Soriano, T. Varanita, R. Quintana-Cabrera, M. Corrado, S. Cipolat, V. Costa, A. Casarin, L.C. Gomes, E. Perales-Clemente, L. Salviati, P. Fernandez-Silva, J.A. Enriquez, and L. Scorrano. 2013. Mitochondrial cristae shape determines respiratory chain supercomplexes assembly and respiratory efficiency. Cell. 155:160-171. doi:10.1016/j.cell.2013.08.032.

Cole, L.W. 2016. The evolution of per-cell organelle number. Front Cell Dev. Biol. 4. doi:10.3389/fcell.2016.00085.

Crompton, M., and A. Costi. 1990. A heart mitochondrial Ca2+-dependent pore of possible relevance to re-perfusion-induced injury. Evidence that ADP facilitates pore interconversion between the closed and open states. Biochem J. 266:33-39. doi:10.1042/bj2660033.

Del Dotto, V., P. Mishra, S. Vidoni, M. Fogazza, A. Maresca, L. Caporali, J.M. McCaffery, M. Cappelletti, E. Baruffini, G. Lenaers, D. Chan, M. Rugolo, V. Carelli, and C. Zanna. 
2017. OPA1 Isoforms in the Hierarchical Organization of Mitochondrial Functions. Cell Rep. 19:2557-2571. doi:10.1016/j.celrep.2017.05.073.

Delettre, C., J.M. Griffoin, J. Kaplan, H. Dollfus, B. Lorenz, L. Faivre, G. Lenaers, P. Belenguer, and C.P. Hamel. 2001. Mutation spectrum and splicing variants in the OPA1 gene. Hum. Genet. 109:584-591. doi:10.1007/s00439-001-0633-y.

Delettre, C., G. Lenaers, J.-M. Griffoin, N. Gigarel, C. Lorenzo, P. Belenguer, L. Pelloquin, J. Grosgeorge, C. Turc-Carel, E. Perret, C. Astarie-Dequeker, L. Lasquellec, B. Arnaud, B. Ducommun, J. Kaplan, and C.P. Hamel. 2000a. Nuclear gene OPA1, encoding a mitochondrial dynamin-related protein, is mutated in dominant optic atrophy. Nature Genetics. 26:207-210. doi:10.1038/79936.

DeVay, R.M., L. Dominguez-Ramirez, L.L. Lackner, S. Hoppins, H. Stahlberg, and J. Nunnari. 2009. Coassembly of Mgm1 isoforms requires cardiolipin and mediates mitochondrial inner membrane fusion. J. Cell Biol. 186:793-803. doi:10.1083/jcb.200906098.

Ehses, S., I. Raschke, G. Mancuso, A. Bernacchia, S. Geimer, D. Tondera, J.-C. Martinou, B. Westermann, E.I. Rugarli, and T. Langer. 2009. Regulation of OPA1 processing and mitochondrial fusion by m-AAA protease isoenzymes and OMA1. J. Cell Biol. 187:1023-1036. doi:10.1083/jcb.200906084.

Elachouri, G., S. Vidoni, C. Zanna, A. Pattyn, H. Boukhaddaoui, K. Gaget, P. Yu-Wai-Man, G. Gasparre, E. Sarzi, C. Delettre, A. Olichon, D. Loiseau, P. Reynier, P.F. Chinnery, A. Rotig, V. Carelli, C.P. Hamel, M. Rugolo, and G. Lenaers. 2011. OPA1 links human mitochondrial genome maintenance to mtDNA replication and distribution. Genome Res. 21:12-20. doi:10.1101/gr.108696.110.

Faelber, K., L. Dietrich, J.K. Noel, F. Wollweber, A.-K. Pfitzner, A. Mühleip, R. Sánchez, M. Kudryashev, N. Chiaruttini, H. Lilie, J. Schlegel, E. Rosenbaum, M. Hessenberger, C. Matthaeus, S. Kunz, A. von der Malsburg, F. Noé, A. Roux, M. van der Laan, W. Kühlbrandt, and O. Daumke. 2019. Structure and assembly of the mitochondrial membrane remodelling GTPase Mgm1. Nature. 571:429-433. doi:10.1038/s41586-0191372-3.

Focusing on mitochondrial form and function. 2018. Nat. Cell Biology. 20:735-735. doi:10.1038/s41556-018-0139-7.

Fonseca, T.B., Á. Sánchez-Guerrero, I. Milosevic, and N. Raimundo. 2019. Mitochondrial fission requires DRP1 but not dynamins. Nature. 570:E34-E42. doi:10.1038/s41586-0191296-y.

Frezza, C., S. Cipolat, O.M. de Brito, M. Micaroni, G.V. Beznoussenko, T. Rudka, D. Bartoli, R.S. Polishuck, N.N. Danial, B.D. Strooper, and L. Scorrano. 2006. OPA1 controls apoptotic cristae remodeling independently from mitochondrial fusion. Cell. 126:177189. doi:10.1016/j.cell.2006.06.025. 
Friedman, J.R., L.L. Lackner, M. West, J.R. DiBenedetto, J. Nunnari, and G.K. Voeltz. 2011. ER tubules mark sites of mitochondrial division. Science. 334:358-362.

doi:10.1126/science.1207385.

Fröhlich, C., S. Grabiger, D. Schwefel, K. Faelber, E. Rosenbaum, J. Mears, O. Rocks, and O. Daumke. 2013. Structural insights into oligomerization and mitochondrial remodelling of dynamin 1-like protein. The EMBO Journal. 32:1280-1292. doi:10.1038/emboj.2013.74.

Gandre-Babbe, S., and A.M. van der Bliek. 2008. The novel tail-anchored membrane protein Mff controls mitochondrial and peroxisomal fission in mammalian Cells. MBoC. 19:24022412. doi:10.1091/mbc.e07-12-1287.

Ge, Y., X. Shi, S. Boopathy, J. McDonald, A.W. Smith, and L.H. Chao. 2020. Two forms of Opa1 cooperate to complete fusion of the mitochondrial inner-membrane. eLife. 9:e50973. doi:10.7554/eLife.50973.

Gilkerson, R.W., E.A. Schon, E. Hernandez, and M.M. Davidson. 2008. Mitochondrial nucleoids maintain genetic autonomy but allow for functional complementation. J. Cell Biol. 181:1117-1128. doi:10.1083/jcb.200712101.

Glater, E.E., L.J. Megeath, R.S. Stowers, and T.L. Schwarz. 2006. Axonal transport of mitochondria requires milton to recruit kinesin heavy chain and is light chain independent. J. Cell Biol. 173:545-557. doi:10.1083/jcb.200601067.

Gomes, L.C., G. Di Benedetto, and L. Scorrano. 2011. During autophagy mitochondria elongate, are spared from degradation and sustain cell viability. Nat. Cell Biol. 13:589-598. doi:10.1038/ncb2220.

Griparic, L., T. Kanazawa, and A.M. van der Bliek. 2007a. Regulation of the mitochondrial dynamin-like protein Opa1 by proteolytic cleavage. J. of Cell Biol. 178:757-764. doi:10.1083/jcb.200704112.

Hales, K.G., and M.T. Fuller. 1997. Developmentally Regulated Mitochondrial Fusion Mediated by a Conserved, Novel, Predicted GTPase. Cell. 90:121-129. doi:10.1016/S00928674(00)80319-0.

Head, B., L. Griparic, M. Amiri, S. Gandre-Babbe, and A.M. van der Bliek. 2009. Inducible proteolytic inactivation of OPA1 mediated by the OMA1 protease in mammalian cells. The J. of Cell Biol. 187:959-966. doi:10.1083/jcb.200906083.

Hoppel, C.L., B. Tandler, H. Fujioka, and A. Riva. 2009. Dynamic organization of mitochondria in human heart and in myocardial disease. Int. J. Biochem. Cell Biol. 41:1949-1956. doi:10.1016/j.biocel.2009.05.004.

Hwa, J.J., M.A. Hiller, M.T. Fuller, and A. Santel. 2002. Differential expression of the Drosophila mitofusin genes fuzzy onions (fzo) and dmfn. Mech. Dev. 116:213-216. doi:10.1016/s0925-4773(02)00141-7. 
Ishihara, N., Y. Eura, and K. Mihara. 2004. Mitofusin 1 and 2 play distinct roles in mitochondrial fusion reactions via GTPase activity. J. of Cell Science. 117:6535-6546.

doi:10.1242/jcs.01565.

Ishihara, N., M. Nomura, A. Jofuku, H. Kato, S.O. Suzuki, K. Masuda, H. Otera, Y. Nakanishi, I. Nonaka, Y. Goto, N. Taguchi, H. Morinaga, M. Maeda, R. Takayanagi, S. Yokota, and K. Mihara. 2009. Mitochondrial fission factor Drp1 is essential for embryonic development and synapse formation in mice. Nat. Cell Biol.. 11:958-966. doi:10.1038/ncb1907.

Ishihara, T., R. Ban-Ishihara, M. Maeda, Y. Matsunaga, A. Ichimura, S. Kyogoku, H. Aoki, S. Katada, K. Nakada, M. Nomura, N. Mizushima, K. Mihara, and N. Ishihara. 2015. Dynamics of mitochondrial DNA nucleoids regulated by mitochondrial fission is essential for maintenance of homogeneously active mitochondria during neonatal heart development. Mol. Cell. Biol. 35:211-223. doi:10.1128/MCB.01054-14.

Karbowski, M., M.M. Cleland, and B.A. Roelofs. 2014. Photoactivatable green fluorescent protein-based visualization and quantification of mitochondrial fusion and mitochondrial network complexity in living cells. Meth. Enzymol. 547:57-73. doi:10.1016/B978-0-12801415-8.00004-7.

Knott, A.B., and E. Bossy-Wetzel. 2008. Impairing the mitochondrial fission and fusion Balance: a new mechanism of neurodegeneration. Ann. N Y Acad. Sci. 1147:283-292. doi:10.1196/annals.1427.030.

Koch, A., M. Thiemann, M. Grabenbauer, Y. Yoon, M.A. McNiven, and M. Schrader. 2003. Dynamin-like protein 1 is involved in peroxisomal fission. J. Biol. Chem. 278:85978605. doi:10.1074/jbc.M211761200.

Kondadi, A.K., R. Anand, S. Hänsch, J. Urbach, T. Zobel, D.M. Wolf, M. Segawa, M. Liesa, O.S. Shirihai, S. Weidtkamp-Peters, and A.S. Reichert. 2020. Cristae undergo continuous cycles of membrane remodelling in a MICOS-dependent manner. EMBO reports. 21:e49776. doi:10.15252/embr.201949776.

Kühlbrandt, W. 2015. Structure and function of mitochondrial membrane protein complexes. BMC Biol. 13. doi:10.1186/s12915-015-0201-x.

Labrousse, A.M., M.D. Zappaterra, D.A. Rube, and A.M. van der Bliek. 1999. C. elegans Dynamin-Related Protein DRP-1 controls severing of the mitochondrial outer membrane. Molecular Cell. 4:815-826. doi:10.1016/S1097-2765(00)80391-3.

Lee, H., S.B. Smith, S.-S. Sheu, and Y. Yoon. 2020a. The short variant of optic atrophy 1 (OPA1) improves cell survival under oxidative stress. J. Biol. Chem. jbc.RA119.010983. doi:10.1074/jbc.RA119.010983.

Lee, H., S.B. Smith, and Y. Yoon. 2017a. The short variant of the mitochondrial dynamin OPA1 maintains mitochondrial energetics and cristae structure. J. Biol. Chem. 292:7115-7130. doi:10.1074/jbc.M116.762567. 
Lee, J.E., L.M. Westrate, H. Wu, C. Page, and G.K. Voeltz. 2016. Multiple dynamin family members collaborate to drive mitochondrial division. Nature. 540:139-143. doi:10.1038/nature20555.

Li, Z., K.-I. Okamoto, Y. Hayashi, and M. Sheng. 2004. The importance of dendritic mitochondria in the morphogenesis and plasticity of spines and synapses. Cell. 119:873887. doi:10.1016/j.cell.2004.11.003.

Liu, X., D. Weaver, O. Shirihai, and G. Hajnóczky. 2009. Mitochondrial "kiss-and-run": interplay between mitochondrial motility and fusion-fission dynamics. EMBO J. 28:3074-3089. doi:10.1038/emboj.2009.255.

Losón, O.C., Z. Song, H. Chen, and D.C. Chan. 2013. Fis1, Mff, MiD49, and MiD51 mediate Drp1 recruitment in mitochondrial fission. $M B o C$. 24:659-667. doi:10.1091/mbc.e12-100721.

Mears, J.A., L.L. Lackner, S. Fang, E. Ingerman, J. Nunnari, and J.E. Hinshaw. 2011. Conformational changes in Dnm1 support a contractile mechanism for mitochondrial fission. Nat. Structural \& Molecular Biology. 18:20-26. doi:10.1038/nsmb.1949.

Menzies, R.A., and P.H. Gold. 1971. The turnover of mitochondria in a variety of tissues of young adult and aged rats. J. Biol. Chem. 246:2425-2429.

Mishra, P., V. Carelli, G. Manfredi, and D.C. Chan. 2014a. Proteolytic cleavage of Opa1 stimulates mitochondrial inner membrane fusion and couples fusion to oxidative phosphorylation. Cell Metabolism. 19:630-641. doi:10.1016/j.cmet.2014.03.011.

Misko, A., S. Jiang, I. Wegorzewska, J. Milbrandt, and R.H. Baloh. 2010. Mitofusin 2 is necessary for transport of axonal mitochondria and interacts with the Miro/Milton complex. J. Neurosci. 30:4232-4240. doi:10.1523/JNEUROSCI.6248-09.2010.

Olichon, A., L. Baricault, N. Gas, E. Guillou, A. Valette, P. Belenguer, and G. Lenaers. 2003. Loss of OPA1 perturbates the mitochondrial inner membrane structure and integrity, leading to cytochrome c release and apoptosis. J. Biol. Chem. 278:7743-7746. doi:10.1074/jbc.C200677200.

Olichon, A., G. Elachouri, L. Baricault, C. Delettre, P. Belenguer, and G. Lenaers. 2007. OPA1 alternate splicing uncouples an evolutionary conserved function in mitochondrial fusion from a vertebrate restricted function in apoptosis. Cell Death Differ. 14:682-692. doi:10.1038/sj.cdd.4402048.

Otera, H., C. Wang, M.M. Cleland, K. Setoguchi, S. Yokota, R.J. Youle, and K. Mihara. 2010. Mff is an essential factor for mitochondrial recruitment of Drp1 during mitochondrial fission in mammalian cells. J. Cell Biol. 191:1141-1158. doi:10.1083/jcb.201007152.

Palmer, C.S., L.D. Osellame, D. Laine, O.S. Koutsopoulos, A.E. Frazier, and M.T. Ryan. 2011. MiD49 and MiD51, new components of the mitochondrial fission machinery. EMBO reports. 12:565-573. doi:10.1038/embor.2011.54. 
Patten, D.A., J. Wong, M. Khacho, V. Soubannier, R.J. Mailloux, K. Pilon-Larose, J.G. MacLaurin, D.S. Park, H.M. McBride, L. Trinkle-Mulcahy, M.-E. Harper, M. Germain, and R.S. Slack. 2014. OPA1-dependent cristae modulation is essential for cellular adaptation to metabolic demand. EMBO J. 33:2676-2691. doi:10.15252/embj.201488349.

Pilling, A.D., D. Horiuchi, C.M. Lively, and W.M. Saxton. 2006. Kinesin-1 and dynein are the primary motors for fast transport of mitochondria in Drosophila motor axons. $M B o C$. 17:2057-2068. doi:10.1091/mbc.e05-06-0526.

Rambold, A.S., B. Kostelecky, N. Elia, and J. Lippincott-Schwartz. 2011. Tubular network formation protects mitochondria from autophagosomal degradation during nutrient starvation. Proc. Natl. Acad. Sci. U.S.A. 108:10190-10195. doi:10.1073/pnas.1107402108.

Ran, F.A., P.D. Hsu, J. Wright, V. Agarwala, D.A. Scott, and F. Zhang. 2013. Genome engineering using the CRISPR-Cas9 system. Nature Protocols. 8:2281-2308. doi:10.1038/nprot.2013.143.

Riva, A., B. Tandler, F. Loffredo, E. Vazquez, and C. Hoppel. 2005. Structural differences in two biochemically defined populations of cardiac mitochondria. Am. J. Physiol. Heart Circ. Physiol. 289:H868-872. doi:10.1152/ajpheart.00866.2004.

Rojo, M., F. Legros, D. Chateau, and A. Lombès. 2002. Membrane topology and mitochondrial targeting of mitofusins, ubiquitous mammalian homologs of the transmembrane GTPase Fzo. J. of Cell Science. 115:1663-1674.

Russo, G.J., K. Louie, A. Wellington, G.T. Macleod, F. Hu, S. Panchumarthi, and K.E. Zinsmaier. 2009. Drosophila Miro is required for both anterograde and retrograde axonal mitochondrial transport. J. Neurosci. 29:5443-5455. doi:10.1523/JNEUROSCI.541708.2009.

Santel, A., and M.T. Fuller. 2001. Control of mitochondrial morphology by a human mitofusin. J. of Cell Science. 114:867-874.

Shi, H., A.J. Rampello, and S.E. Glynn. 2016a. Engineered AAA+ proteases reveal principles of proteolysis at the mitochondrial inner membrane. Nat. Commun. 7:13301. doi:10.1038/ncomms13301.

Shi, H., A.J. Rampello, and S.E. Glynn. 2016b. Engineered AAA+ proteases reveal principles of proteolysis at the mitochondrial inner membrane. Nat. Commun. 7. doi:10.1038/ncomms13301.

Smirnova, E., L. Griparic, D.-L. Shurland, and A.M. van der Bliek. 2001. Dynamin-related protein Drp1 is required for mitochondrial division in mammalian cells. MBoC. 12:22452256. doi:10.1091/mbc.12.8.2245. 
Song, Z., H. Chen, M. Fiket, C. Alexander, and D.C. Chan. 2007a. OPA1 processing controls mitochondrial fusion and is regulated by mRNA splicing, membrane potential, and Yme1L. J. Cell Biol. 178:749-755. doi:10.1083/jcb.200704110.

Song, Z., M. Ghochani, J.M. McCaffery, T.G. Frey, and D.C. Chan. 2009. Mitofusins and OPA1 mediate sequential steps in mitochondrial membrane fusion. Mol Biol Cell. 20:35253532. doi:10.1091/mbc.E09-03-0252.

Stowers, R.S., L.J. Megeath, J. Górska-Andrzejak, I.A. Meinertzhagen, and T.L. Schwarz. 2002. Axonal transport of mitochondria to synapses depends on milton, a novel Drosophila protein. Neuron. 36:1063-1077. doi:10.1016/s0896-6273(02)01094-2.

Sun, M.G., J. Williams, C. Munoz-Pinedo, G.A. Perkins, J.M. Brown, M.H. Ellisman, D.R. Green, and T.G. Frey. 2007. Correlated three-dimensional light and electron microscopy reveals transformation of mitochondria during apoptosis. Nat. Cell Biol. 9:1057-1065. doi:10.1038/ncb1630.

Takeyama, N., N. Matsuo, and T. Tanaka. 1993. Oxidative damage to mitochondria is mediated by the Ca2+-dependent inner-membrane permeability transition. Biochem. J. 294:719725. doi:10.1042/bj2940719.

Tanaka, A., M.M. Cleland, S. Xu, D.P. Narendra, D.-F. Suen, M. Karbowski, and R.J. Youle. 2010. Proteasome and p97 mediate mitophagy and degradation of mitofusins induced by Parkin. J. Cell Biol. 191:1367-1380. doi:10.1083/jcb.201007013.

Tilokani, L., S. Nagashima, V. Paupe, and J. Prudent. 2018. Mitochondrial dynamics: Overview of molecular mechanisms. Essays in Biochemistry. 62:341-360. doi:10.1042/EBC20170104.

Tondera, D., S. Grandemange, A. Jourdain, M. Karbowski, Y. Mattenberger, S. Herzig, S. Da Cruz, P. Clerc, I. Raschke, C. Merkwirth, S. Ehses, F. Krause, D.C. Chan, C. Alexander, C. Bauer, R. Youle, T. Langer, and J.-C. Martinou. 2009a. SLP-2 is required for stressinduced mitochondrial hyperfusion. EMBO J. 28:1589-1600. doi:10.1038/emboj.2009.89.

Twig, G., A. Elorza, A.J.A. Molina, H. Mohamed, J.D. Wikstrom, G. Walzer, L. Stiles, S.E. Haigh, S. Katz, G. Las, J. Alroy, M. Wu, B.F. Py, J. Yuan, J.T. Deeney, B.E. Corkey, and O.S. Shirihai. 2008. Fission and selective fusion govern mitochondrial segregation and elimination by autophagy. EMBO J. 27:433-446. doi:10.1038/sj.emboj.7601963.

Verstreken, P., C.V. Ly, K.J.T. Venken, T.-W. Koh, Y. Zhou, and H.J. Bellen. 2005. Synaptic Mitochondria are critical for mobilization of reserve pool vesicles at Drosophila neuromuscular junctions. Neuron. 47:365-378. doi:10.1016/j.neuron.2005.06.018.

Wai, T., J. García-Prieto, M.J. Baker, C. Merkwirth, P. Benit, P. Rustin, F.J. Rupérez, C. Barbas, B. Ibañez, and T. Langer. 2015. Imbalanced OPA1 processing and mitochondrial fragmentation cause heart failure in mice. Science. 350:aad0116. doi:10.1126/science.aad0116. 
Yamaguchi, R., L. Lartigue, G. Perkins, R.T. Scott, A. Dixit, Y. Kushnareva, T. Kuwana, M. Ellisman, and D.D. Newmeyer. 2008. Opa1-mediated cristae opening is Bax/Bak- and BH3-dependent, required for apoptosis, and independent of Bak oligomerization. Mol. Cell. 31:557-569. doi:10.1016/j.molcel.2008.07.010.

Zhao, J., T. Liu, S. Jin, X. Wang, M. Qu, P. Uhlén, N. Tomilin, O. Shupliakov, U. Lendahl, and M. Nistér. 2011. Human MIEF1 recruits Drp1 to mitochondrial outer membranes and promotes mitochondrial fusion rather than fission. The EMBO J.. 30:2762-2778. doi:10.1038/emboj.2011.198. 


\title{
Chapter 2
}

\section{Identification of new OPA1 cleavage site reveals that short isoforms regulate mitochondrial fusion}

\author{
Ruohan Wang ${ }^{1}$, Prashant Mishra ${ }^{2}$, Spiros D. Garbis ${ }^{3}$, Annie Moradian ${ }^{3}$, Michael J. Sweredoski ${ }^{3}$, \\ and David C. Chan ${ }^{1 *}$
}

From the ${ }^{1}$ Division of Biology and Biological Engineering, California Institute of Technology, Pasadena, CA 91125; ${ }^{2}$ Children's Medical Center Research Institute, University of Texas Southwestern Medical Center, Dallas, TX 75390, USA; Department of Pediatrics, University of Texas Southwestern Medical Center, Dallas, TX 75390, USA; The Green Center for Systems Biology, University of Texas Southwestern Medical Center, Dallas, TX 75390; ${ }^{3}$ Proteome Exploration Laboratory of the Beckman Institute, California Institute of Technology, Pasadena, CA 91125. 


\begin{abstract}
OPA1, a large GTPase of the dynamin superfamily, mediates fusion of the mitochondrial inner membranes, regulates cristae morphology, and maintains respiratory chain function. Innermembrane-anchored long forms of OPA1 (1-OPA1) are proteolytically processed by the OMA1 or YME1L proteases, acting at cleavage sites S1 and S2, respectively, to produce short forms (sOPA1). In both mouse and human, half of the mRNA splice forms of Opal are constitutively processed to yield exclusively s-OPA1. However, the function of s-OPA1 in mitochondrial fusion has been debated, because in some stress conditions, s-OPA1 is dispensable for fusion. By constructing cells in which the Opal locus no longer produces transcripts with $\mathrm{S} 2$ cleavage sites, we generated a simplified system to identify the new YME1L-dependent site S3 that mediates constitutive and complete cleavage of OPA1. We show that mitochondrial morphology is highly sensitive to the ratio of 1-OPA1 to s-OPA1, indicating that s-OPA1 regulates mitochondrial fusion.
\end{abstract}




\section{Introduction}

By controlling mitochondrial form and function, the dynamics of mitochondrial fusion and fission regulate cellular physiology, including responses to metabolic demands and cellular stress (Chan, 2019). As double-membrane organelles, mitochondria fuse by a two-step process coordinated by a set of dynamin superfamily GTPases. In mammalian cells, mitochondrial outer membrane fusion mediated by the mitofusins MFN1 and MFN2 is followed by inner membrane fusion mediated by OPA1 (Chan, 2019). Each of these fusion molecules is essential for embryonic development in mouse (Chen et al., 2003b; Alavi et al., 2007). In humans, heterozygous mutations in MFN2 and OPA1 cause the neurodegenerative diseases CharcotMarie-Tooth Disease 2A and Autosomal Dominant Optic Atrophy, respectively (Alexander et al., 2000a; Delettre et al., 2000b; Chan, 2019).

Due to alternative splicing and post-translational processing, the OPA1 locus gives rise to a complicated set of protein isoforms. In humans, OPA1 encodes for $8 \mathrm{mRNA}$ splice forms that are generated by alternative splicing of exons 4, 4b, and 5b (Fig. S2.1A). The encoded OPA1 polypeptides are directed into mitochondria due to an $\mathrm{N}$-terminal mitochondrial targeting sequence (MTS) that is cleaved by the mitochondrial processing peptidase (MPP) soon after entry of this region into the matrix. Lateral exiting of the polypeptide from the TIM (translocase of the inner membrane) pore results in a "long" form of OPA1 (1-OPA1) that is anchored in the mitochondrial inner membrane by an N-terminal transmembrane segment. A fraction of 1-OPA1 is proteolytically cleaved beyond the transmembrane segment by either OMA1 or YME1L, inner membraneanchored proteases, to produce slightly different, non-membrane-anchored, "short" forms (sOPA1). OMA1 cleaves at the S1 site encoded by exon 5 (Head et al., 2009; Ehses et al., 2009), and YME1L cleaves at the S2 site encoded by the alternative exon 5b (Song et al., 2007b; Griparic 
et al., 2007b). For isoforms 1, 2, 4, and 7, the moderate action of these proteases typically results in roughly comparable levels of long versus short OPA1. In contrast, the polypeptides encoded by the other 4 mRNA splice forms $(3,5,6$, and 8), which all contain exon $4 \mathrm{~b}$, are such avid substrates for proteolytic processing that they generate only short forms (Song et al., 2007b; Griparic et al., 2007b) (Fig. S2.1A). Although little detail is available, it has been suggested that exon $4 \mathrm{~b}$ may encode a protease cleavage site (Head et al., 2009; Olichon et al., 2007; Lee et al., 2017b). Under normal conditions, a balanced combination of long and short forms is required to maintain high levels of mitochondrial fusion (Song et al., 2007b). Proteolytic processing of the OPA1 ortholog Mgm1p is also necessary for normal levels of mitochondrial fusion in yeast cell (DeVay et al., 2009). In contrast, either s-OPA1 or 1-OPA1 is sufficient for the non-fusion functions of OPA1, such as maintenance of mtDNA, cristae structure, and respiratory chain assembly (Del Dotto et al., 2017; Lee et al., 2017).

These observations raise several important issues. How do long and short forms of OPA1 contribute to mitochondrial inner membrane fusion? In particular, what is the purpose of the short forms of OPA1, given that 4 of 8 mRNA splice isoforms give rise to exclusively short forms? There are currently two general models explaining how long and short OPA1 isoforms regulate inner membrane fusion activity. The first model is that a mixture of long and short forms is critical for fusion (Song et al., 2007; Mishra et al., 2014). This model is based on cell culture observations that expression of 1-OPA1 or s-OPA1 alone results in very low mitochondrial fusion activity and that robust levels of fusion require a combination of both long and short forms (Song et al., 2007b). Furthermore, in an in vitro fusion assay, mitochondria containing 1-OPA1 do not fuse until partial proteolytic cleavage is triggered (Mishra et al., 2014b). The second model is that 1-OPA1 and sOPA have distinct activities, with 1-OPA1 being the only fusogenic form. This model is based on 
the observation that cells lacking OMA1 and YME1L, which produce only long forms of OPA1, nevertheless show tubular mitochondria and mitochondrial fusion activity (Anand et al., 2014b). In addition, under a stress condition termed stress-induced mitochondrial hyperfusion, 1-OPA1 is sufficient for fusion activity (Tondera et al., 2009b). These observations indicate that under specific cellular conditions, 1-OPA1 alone can have substantial fusion activity, even though this does not appear to be the case under basal conditions. S-OPA1 has instead been suggested to have fission activity, based on the observation that overexpression of s-OPA1 leads to mitochondrial fragmentation (Anand et al., 2014b). Recent reconstitution of OPA1 fusion activity in vitro (Ban et al., 2017b) has not distinguished between these two models, because both 1-OPA1 and s-OPA1 show liposome fusion activity (Ban et al., 2018a). However, consistent with the first model, sOPA1 seems to work synergistically with 1-OPA1 to increase in vitro fusion activity (Ban et al., 2017b).

To understand the role of the short forms of OPA1, we used CRISPR-Cas9 gene editing to generate cells and mice lacking OPA1 isoforms containing the YME1L-dependent S2 site encoded by exon $5 b$. This genetic change eliminated all OPA1 isoforms containing this exon, and we anticipated that the role of YME1L in regulating OPA1 function would be abrogated due to the elimination of the YME1L cleavage site. Surprisingly, however, OPA1 processing was still regulated by YME1L due to a novel cleavage site, that we term S3, encoded by the alternative exon $4 \mathrm{~b}$. Notably, isoform 5 contains exon $4 \mathrm{~b}$ and is constitutively cleaved at $\mathrm{S} 3$, and therefore this mRNA isoform contributes only s-OPA1 to the OPA1 repertoire. In specific cellular contexts, we show that depletion of S3-generated s-OPA1 causes mitochondrial fragmentation, and conversely, that increased expression of S3-generated s-OPA1 induces mitochondrial elongation. 
These results demonstrate that s-OPA1 works with 1-OPA1 to tune the level of mitochondrial fusion.

\section{RESULTS}

\section{OMA1 and YME1L differentially modulate OPA1 fusion activity}

Whereas human OPA1 has 8 different mRNA splice variants (Fig. S2.1A), mouse tissues express only four-isoforms 1, 5, 7, and 8 (Akepati et al., 2008) (Fig. 2.1A). Isoforms 1 and 7 each produce a mixture of 1-OPA1 and one or two versions of s-OPA1, respectively. The short isoforms are produced by proteolytic cleavage of the long isoform by the mitochondrial inner membrane proteases OMA1 (at the S1 site) and YME1L (at the S2 site). In contrast, proteolytic processing of isoforms 5 and 8 goes to completion and results in only short isoforms (Song et al., 2007b).

In mouse embryonic fibroblasts (MEFs), this group of 4 mRNA isoforms gives rise to five protein bands when cell lysates are resolved on SDS-PAGE (labeled $a$-e in Fig. 2.1A, B). Bands $a$ and $b$ are the long forms arising from isoform 7 and isoform 1, respectively (Fig. 2.1A). Bands $c$ and $d$ are S1- and S2-cleavage products of isoform 7 generated by OMA1 and YME1L, respectively. Band $e$ is the short, S1-cleaved form of isoform 1. Isoforms 5 and 8 generate only short isoforms that would be expected to contribute to bands $c$-e (Song et al., 2007b). The same set of OPA1 bands has been observed in other studies, although the relative intensities can vary, perhaps due to differences in culture conditions (Anand et al., 2014b).

The dependencies of bands $c-e$ on the OMA1 or YME1L proteases were confirmed by the OPA1 band pattern in lysates from Omal-null or Ymell-null cells (Fig. 2.1B). The OPA1 band patterns observed in the protease mutant cells are tabulated in Fig. S2.1B. The identities of the Omal-null and YmelL-null cells were confirmed by Western blotting against the relevant 
proteases (Fig. S2.1C). Notably, there is a longer band emerging in both the Ymell-null and Yme1//Omal-null cells, likely due to the disruption of YME1L-dependent short isoform formation (i.e., isoform 8).

To understand the role of these proteases in regulating OPA1 fusogenic function, we examined Omal/Ymell double knockout MEFs, which contain only long forms of OPA1 (Anand et al., 2014b). In standard high glucose culture medium, these cells show increased mitochondrial fragmentation compared to wildtype (WT) cells (Fig. 2.1C, D). Many Oma1/Yme1l mutant cells nevertheless show short tubular mitochondria (Fig. 2.1D), so the degree of mitochondrial fragmentation is substantially less severe than in $M f n 1 / M f n 2$-null or Opal-null cells (Chen et al., 2005a, 2007). Omal-null MEFs show normal mitochondrial morphology profiles, and Ymell-null MEFs show highly fragmented mitochondria (Fig. 2.1D). The overall trends - that mitochondria in Oma1/Yme1l mutant cells are more fragmented than in WT cells, but less than in Ymell mutant cells - are consistent with a previous study (Anand et al., 2014). OPA1 band patterns are similar under the different media conditions (Fig. S2.1D).

We tested how these mutant cells respond to culture conditions that increase the level of mitochondrial fusion. Cultured MEFs increase mitochondrial fusion activity and show mitochondrial elongation when cultured in medium that induces oxidative phosphorylation (OXPHOS). This response has been shown to depend on YME1L activity (Mishra et al., 2014b). Consistent with this idea, we found mitochondrial elongation caused by OXPHOS-inducing medium to be abrogated in Ymell-null and Omal/Ymell-null MEFs (Fig. 2.1C, D). As measured in the photo-activatable (PA)-GFP fusion assay, mitochondrial fusion under basal conditions was substantially reduced in Omal/Yme1-null MEFs compared to WT cells and did not increase in response to OXPHOS-inducing medium (Fig. 2.1E). In contrast, cells of all genotypes tested in 
this study show robust mitochondrial elongation in response to cycloheximide (CHX) treatment, which causes stress-induced mitochondrial hyperfusion (Tondera et al., 2009b) (Fig. 2.1C, D, E). Hyperfusion is induced rapidly after the addition of CHX (Fig. 2.1E). CHX treatment enhanced PA-GFP signal dilution in both WT and Oma1/Yme1l-null cells. WT cells showed a substantially greater fusion rate than the latter, indicating the reduced fusion capability in $O m a 1 / Y m e 1 l$-null cells even in the presence of a strong fusion stimulus. Taken together, our observations agree with the previous finding that Omal/Ymell-null cells, containing only 1-OPA1, retain mitochondrial fusion activity (Anand et al., 2014b). However, the level of fusion activity is substantially lower compared to WT cells. The OMA1 and YME1L proteases differentially regulate OPA1 function. Stress-induced hyperfusion does not depend on either protease, consistent with the report that 1OPA1 is sufficient in mediating fusion under this stress condition (Tondera et al., 2009b). In contrast, OXPHOS-induced mitochondrial elongation is dependent on YME1L activity, as noted previously (Mishra et al., 2014b).

\section{The Opa1 S2 site is necessary for mediating OXPHOS-induced fusion in vivo}

To clarify the functions of YME1L-dependent cleavage of OPA1, we used CRISPR-Cas9mediated gene targeting in MEFs to generate mutations in the Opal genomic locus at exon 5b, which encodes the S2 cleavage site (Fig. 2.2A, B). We hoped to generate deletions in exon $5 \mathrm{~b}$ that would disrupt the Opal reading frame, resulting in a downstream premature stop codon that would prevent the formation of functional OPA1 isoforms containing S2. In this scenario, the mutant cells would be expected to have only long isoform 1 (band $b$ ) and the corresponding S1 cleavage product (band $e$ ). Bands $a, c$, and $d$ would be missing, because those arise from exon 5 b-containing transcripts (Fig. 2.2B). 
After the transient expression of Cas9 and sgRNA against the exon 5b target in MEFs, we identified multiple colonies that lacked bands $a, c$, and $d$ (Fig. 2.2C). To ensure against clonespecific effects, we used three or more $\Delta$ exon $5 b$ clones in all our experiments, and results from two different clones are shown in the figures. DNA sequencing showed that clone 1 has a homozygous two-nucleotide deletion, and clone 2 has two longer deletions that each cause frameshifting (Fig. 2.2A).

The $\Delta$ exon $5 b$ cells showed normal mitochondrial profiles when grown in a regular glucose-containing medium. In addition, they showed a normal stress-induced hyperfusion response, dramatically elongating mitochondria upon $\mathrm{CHX}$ stimulation (Fig. 2.2D and Fig. S2.2A). In OXPHOS-inducing medium, however, the mutant cells did not show any elongation of mitochondria compared to growth in control medium, indicating that S2-cleavage of Opa1 is required for OXPHOS-induced hyperfusion. Although OXPHOS-inducing medium does not promote mitochondrial elongation in $\Delta$ exon $5 b$ cells, we did not detect physiological consequences. The OXPHOS-inducing medium caused upregulation of oxygen consumption in these cells (Fig. S2.2B), indicating that the increase in respiratory function is not dependent on mitochondrial elongation. Moreover, both WT cells and $\Delta$ exon $5 b$ cells showed similar cell growth after switching to the OXPHOS-inducing medium (Fig. S2.2C). In addition to MEFs, we generated two $\Delta$ exon $5 b$ mouse lines. Western analysis of mutant tissues showed abrogation of Opal exon 5b-containing protein bands (Fig. S2.2D). Despite this biochemical change in OPA1 isoforms, the mutant mice showed no obvious physiological dysfunction and gained weight normally (Fig. S2.2E) for at least one year of age. 


\section{Detection of a new OPA1 cleavage site in WT and $\Delta$ exon $5 b$ cells}

In the course of this study, we switched to a higher resolution gel system to study these OPA1 protein isoforms. With this new system, what was previously designated as band $d$ in WT cells could be resolved into doublet bands, which we designate as $d$ and $d^{\prime}$ (Fig. 2.3A). Upon reanalysis, we realized that $\Delta$ exon $5 b$ cells produce two short Opal bands, $d$ ' and $e$. With the deletion of the YME1L-dependent S2 site, $\Delta$ exon $5 b$ cells would be expected to show only OMA1mediated processing of OPA1. In $\Delta$ exon 5b/Omal-null cells, band $e$ is missing, but $d$ ' band remains, indicating that it is OMA1-independent (Fig. 2.3A).

Because YME1L is the other known intermembrane protease involved in OPA1 posttranslational processing, we used shRNA to test whether it is essential for the production of band $d^{\prime}$. The knockdown efficiencies of the Ymell and Omal shRNAs were confirmed by Western blotting (Fig. S2.3A, B). In WT cells, knockdown of YME1L caused the disappearance of band $d$ and $d^{\prime}$, whereas knockdown of OMA1 caused the disappearance of bands $c$ and $e$ (Fig. 2.3B). The dependence of $d$ ' on YME1L but not OMA1 was further confirmed in both $\Delta$ exon 5b/Omal-null cells and $\Delta$ exon $5 b$ cells (Fig. 2.3B).

To identify the source of the new short OPA1 band $d$ ', we used PCR analysis of cDNA from $\Delta$ exon $5 b$ cells to identify the major remaining Opal mRNA transcripts. Using primers flanking the alternative splicing exons $4,4 \mathrm{~b}$, and $5 \mathrm{~b}$ to distinguish individual mRNA transcripts (Fig. S2.3C), we found that the mutant cells expressed mRNA splice variants 1 and 5 (confirmed both by PCR analysis and sequencing), with isoform 1 being slightly more abundant (Fig. 2.3C, D). It is not surprising that isoforms 7 and 8 are missing, because they contain exon $5 \mathrm{~b}$. Isoform 5 instead contains the alternative exon $4 \mathrm{~b}$ (Fig 2.3D), and like other exon 4b-containing isoforms, was previously shown to be constitutively cleaved into a short isoform (Song et al., 2007b). The 
constitutive cleavage of isoform 5 was assumed to occur at the S1 site, the only known cleavage site in this isoform (Song et al., 2007b). However, we wondered whether this assumption might be incorrect, because cleavage of isoform 5 at $\mathrm{S} 1$ would lead to band $e$, not $d$ '. To test whether isoform 5 could generate band $d$ ', we expressed isoform 5 in Opal-null cells. Isoform 5 generated a short isoform that was clearly distinct from $e$ and co-migrated with the $d$ ' bands present in $\Delta e x o n 5 b$ and Aexon 5b/Oma1-null cells (Fig. 2.3E). This observation indicates that isoform 5 is the likely source of band $d^{\prime}$. Its cleavage site is $\mathrm{N}$-terminal to $\mathrm{S} 1$, as indicated by the slightly larger size of $d^{\prime}$ compared to $e$.

To determine the protease responsible for cleavage of isoform 5, and further confirm that the YME1L-dependent band $d$ ' indeed arises from isoform 5, we compared the behavior of Cterminal FLAG-tagged human isoforms 5 and 1 in mutant MEFs lacking OMA1 or YME1L. Consistent with previous studies showing cleavage at S1 by OMA1, isoform 1-FLAG produces a short isoform that is dependent on OMA1 (Fig. 2.3F). When YME1L is absent, more of this short isoform is produced, presumably due to upregulation of OMA1 activity (Wai et al., 2015). As expected, isoform 5-FLAG is completely processed in control MEFs. The absence of OMA1 does not affect the cleavage pattern, indicating that the production of isoform 5 short form is independent of OMA1 (Fig. 2.3F). The removal of Yme $1 l$ completely abrogates production of this short form and results in a lower band that co-migrates with the short isoform generated from isoform 1 (Fig. 2.3F), as well as a small amount of long form. These results indicate that, under normal conditions, isoform 5 is processed exclusively at a site upstream of S1, in a YME1Ldependent manner, to produce band $d^{\prime}$. When YME1L is absent, cleavage is activated at the downstream S1 site. Isoform 5 produces only long form when both OMA1 and YME1L are absent (Fig. 2.3F, last lane). Together, the results suggest that OPA1 isoform 5 is highly susceptible to 
protease processing, normally by YME1L. OMA1 is also capable of processing isoform 5 into S1cleaved OPA1 in the absence of YME1L. No additional proteases are sufficient for isoform 5 cleavage, as indicated by the formation of long isoform 5 when both Yme1l and Omal are absent.

\section{Identification of a leucine-rich, YME1L-dependent cleavage site in exon 4b}

Our results so far suggest that isoform 5 contains a novel YME1L-dependent cleavage site upstream of S1. To pinpoint the new cleavage site (which we designate as S3), we asked if cleavage is dependent on exon $4 \mathrm{~b}$, the first exon upstream of exon 5 . Knockdown of exon $4 \mathrm{~b}$ with shRNA substantially reduced the intensity of band $d^{\prime}$ in WT, $\Delta$ exon $5 b$, and $\Delta$ exon $5 b / O m a l$-null cells (Fig. 2.4A, band d' marked with asterisks). This dependence suggests that the cleavage site likely resides in exon $4 \mathrm{~b}$. To test this idea, we analyzed the behavior of a series of isoform 5 mutants (Mut 1-9) containing various short deletions within exon 4b (Fig. 2.4B). Mutants 1, 2, and 3, containing deletions in the 5 ' half of exon $4 \mathrm{~b}$, show no defect in isoform 5 cleavage processing, indicating that the first half of exon $4 \mathrm{~b}$ does not contain the cleavage site (Fig. 2.4C). Mutations 4, 5, and 6 result in small amounts of the long form, which normally is absent. In addition, these mutants show prominent production of band $e$. Mutants 7 and 8 also show presence of the long form and band $e$. In addition, these mutants show novel cleavage products forming a smear above band $e$ (Fig. 2.4C). Mutation 9, which deleted the bridging amino acids between exon 4b and 5, generates band $e$ and produces new cleavage products without generating a long form of isoform 5. These results suggest that S3 cleavage is sensitive to mutations in the 3' half of exon $4 \mathrm{~b}$, and particularly to mutations (mutants 7 and 8) affecting a stretch of 5 consecutive leucines (amino acids 199-203 in isoform 5). Interestingly, the most prominent effects of the mutations are in appearance of the long form and novel cleavage products, rather than in elimination of band $d^{\prime}$. 
To determine the proteases responsible for cleavage of the mutants, we expressed the FLAG-tagged mutants in protease mutant MEFs (Fig. 2.4D). In mutants 6 and 7, the smallest cleavage product disappeared in Omal-null cells, indicating that it is OMA1-dependent. In mutant 7, there are larger short forms that are YME1-dependent. In the absence of both OMA1 and YME1L, no cleavage was observed, demonstrating that these two proteases are essential for isoform 5 processing.

To identify the cleavage site more precisely, we used tandem mass spectrometry to determine the N-terminal sequence of band $d$, produced by overexpression of FLAG-tagged human isoform 5 in 293 T cells. Band d' was collected by immunoprecipitation against FLAG tag, resolved on SDS-PAGE, and isolated for biochemical analysis. Peptides were generated by trypsin digestion and identified by liquid chromatography coupled with nanospray ionization tandem mass spectrometry (LC-MS). Of the peptides identified, only three peptides had non-tryptic N-termini.

The two predominant ones had N-termini located within exon $4 \mathrm{~b}$, beginning with the second to last leucine and last leucine of the penta-leucine stretch (Fig 2.4E). The latter peptide had by far the highest intensity, suggesting that it represents the predominant S3 cleavage product. To test this idea, we designed isoform 5 mutants (mutants 10-13) in which the last 1-3 leucines of the penta-leucine stretch were substituted with alanine. We found that substitution of a single leucine (mutants 12 and 13) had little effect on band $d^{\prime}$ production, though a small level of larger cleavage products could be observed (Fig. 2.4F). When both of the last two leucines were substituted (mutant 11), there was a more substantial level of aberrant cleavage, and the long isoform appeared. Both these effects were increased when the last three leucines were substituted (mutant 10). Taken together, the LC-MS and mutational analysis strongly argue that the S3 cleavage site lies within the penta-leucine stretch encoded by the end of exon $4 \mathrm{~b}$. Although the 
product ion spectra indicated that cleavage primarily occurs $\mathrm{N}$-terminal to the last leucine, the mutational phenotypes suggest that cleavage can occur at other locations very near the pentaleucine stretch (Fig. 2.4C, lane 9-13). None of the mutations completely eliminate production of band $d^{\prime}$, suggesting that cleavage can still occur very close to $\mathrm{S} 3$ when the site is mutated. It is also apparent that isoform 5 is highly prone to proteolytic processing-when the S3 site is mutated, cleavage is activated at $\mathrm{S} 1$ or at cryptic sites $\mathrm{N}$-terminal to $\mathrm{S} 3$, leading to variant cleaved forms of isoform 5. Our analysis indicates that $\Delta e x o n 5 b$ cells retain two dominant Opa1 mRNA transcripts, isoforms 1 and 5. This simplified transcript profile results in one 1-OPA1 (from isoform 1) and two s-OPA1 (isoform 1/S1 and isoform 5/S3) (Fig. 2.4G).

\section{The S3-cleaved short form of isoform 5 regulates mitochondrial tubulation}

In both humans and mice, half of the Opal splice forms contain exon $4 \mathrm{~b}$ and are constitutively cleaved to yield exclusively short isoforms (Song et al., 2007b). This raises the issue of why Opa1 encodes multiple mRNA splice forms whose only apparent purpose is to generate short isoforms. We used isoform 5 to address this issue. Consistent with previous studies (Song et al., 2007b), isoform 5 has almost no mitochondrial elongation activity when expressed in Opa1null MEFs, which have highly fragmented mitochondria due to the complete loss of inner membrane fusion (Fig. 2.5A, S5A, and S4A). Isoform 5 mutations that disrupt S3 processing, however, show substantial mitochondrial fusion activity, as indicated by their ability to elongate mitochondria (Fig. 2.5A, S5A and S4A, B). Because these mutants produce a variety of short forms as well as a long form, these results fit with the model that a mixture of long and short isoforms of OPA1 is important for mitochondrial fusion activity (Song et al., 2007b). Alternatively, these 
results could be explained by proposing that it is the production of the long isoform that induces mitochondrial fusion activity.

To distinguish between these two models, we further probed the physiological function of the short form of isoform 5 . We used shRNA to knock down exon $4 \mathrm{~b}$ in $\Delta$ exon $5 b$ and $\Delta$ exon 5b/Omal-null cells, which have a simplified portfolio of Opa1 mRNA splice forms. As expected, knockdown of exon $4 \mathrm{~b}$ resulted in reduction of band d' in both cell lines (Fig. 2.4A, Fig 2.5B). In the $\triangle$ exon $5 \mathrm{~b}$ MEFs, exon $4 \mathrm{~b}$ knockdown leads to dramatic mitochondrial fragmentation (Fig. 2.5C, Fig. S2.5B). Similarly, in $\Delta$ exon 5b/Omal-null cells, knockdown of exon 4b results in mitochondrial fragmentation (Fig. 2.5C, Fig. S2.5B). To verify that the knockdown result is not due to off-target effects, we re-expressed isoform 5 in the exon $4 \mathrm{~b}$ knockdowns (Fig. 2.5B) and observed elongation of mitochondrial morphology in both cell lines (Fig. 2.5C, Fig. S2.5B).

To further test this idea that a short form of OPA1 can collaborate with a long form to regulate mitochondrial fusion, we developed an expression system to co-express two different OPA1 splice forms in Opa1-null MEFs. We constructed a bicistronic expression vector to generate isoform 1 along with isoform 5 or isoform 1 along with control YFP. Western blot confirmed the expected OPA1 band pattern in these cells (Fig. 2.5D). With isoform 1 expression alone, the long band $\mathrm{b}$ and the short band e were produced. With bicistronic expression of isoform 1 and isoform 5, an additional short form (d' from isoform 5) was added. This dual expression recapitulated the bands observed in $\Delta$ exon $5 \mathrm{~b}$ cells (Fig. 2.5D) and resulted in longer mitochondrial profiles. Significantly more cells contained interconnected mitochondria, and fewer cells had fragmented mitochondria, compared to expression of isoform 1 alone (Fig. 2.5E, Fig. S2.5C). These results suggest that S3-cleaved s-OPA1 can work with 1-OPA1 to promote mitochondrial fusion. To determine whether this synergy requires GTP hydrolysis activity in s-OPA1, we used the 
bicistronic system to express wildtype isoform 1 with isoform 5 containing a dysfunctional GTPase domain (iso 5-G300E) in Opa1-null MEFs. The protein band pattern was similar in cells expressing Iso 1-IRES-Iso 5-G300E compared to cells expressing Iso 1-IRES-Iso 5 (Fig. S2.5D). The mutated isoform 5 was ineffective at promoting mitochondrial fusion in combination with wildtype isoform 1, demonstrating that GTPase activity is required in s-OPA1 to maintain fusion function (Fig. S2.5 C, E). 


\section{DISCUSSION}

The OPA1 locus has several mechanisms that safeguard the production of s-OPA1. Of the 8 OPA1 mRNA isoforms in humans, 4 contain exon 4 b (Delettre et al., 2001) and encode polypeptides that are fully processed to s-OPA1 (Song et al., 2007b). It remains to be determined why inclusion of exon $4 \mathrm{~b}$ causes such extensive processing by YME1L. A short hydrophobic sequence (FAWFP) has been found to act as a degron for YME1L (Shi et al., 2016a). However, no similar sequences are apparent in exon $4 \mathrm{~b}$ or the rest of OPA1. In the mouse, only 4 mRNA isoforms are expressed (Akepati et al., 2008), but similar to humans, half of them contain exon 4b and produce only s-OPA1. Moreover, with our identification of S3, there are now three cleavage sites that result in the production of s-OPA1 (Fig. 2.5F). This apparent redundancy of sites ensures production of s-OPA1 when one mechanism is disrupted. There is evidence that s-OPA1 is important for cell health, especially under conditions of oxidative stress (Lee et al., 2020b).

The redundancy of two YME1L-dependent sites (S2 and S3) likely explains why mice lacking the S2 site do not show obvious physiological abnormalities. In $\Delta$ exon $5 \mathrm{~b}$ mice and cells, RNA isoforms retaining the S3 site are still present and produce sufficient levels of YME1dependent s-OPA1 to maintain cellular function. In addition to redundancy of S2 and S3, there appears to be crosstalk between sites to ensure cleavage of OPA1 precursors. With isoform 5, for example, mutation of the S3 site does not abrogate proteolytic processing of OPA1. Rather, it activates OMA1-dependent cleavage at S1 and YME1L-dependent cleavage at upstream cryptic sites, resulting in new short isoforms (Fig. 2.5F). Similarly, mutation of the $\mathrm{S} 1$ site activates cryptic cleavage sites (Song et al., 2007b). The presence of these multiple mechanisms to generate s-OPA1 suggests that the processed forms likely play an important functional role. It is known that s-OPA1 alone is functional for maintenance of mitochondrial DNA, respiratory complexes, and cristae 
structure (Lee et al., 2017c; Del Dotto et al., 2017a), but its role in modulating mitochondrial fusion has been less clear.

Our results support a model in which s-OPA1 collaborates with 1-OPA1 to regulate mitochondrial inner membrane fusion. This principle can be readily observed in our engineered cells that have various altered combinations of OPA1 isoforms. In $\Delta$ exon 5b/Oma1-null cells, only one long form and one short form are produced. Further reduction of s-OPA1 in these cells causes dramatic mitochondrial fragmentation (Fig. 2.5C). Although the long forms by themselves can have fusion activity under certain contexts (mainly the stress-induced hyperfusion condition) (Tondera et al., 2009b), 1-OPA1 alone is ineffective at maintaining tubular mitochondrial morphology after exon $4 \mathrm{~b}$ is knocked down in exon 5b/Oma1-null cells, which then produce only the long form of isoform 1 (band b). Similarly, cells expressing both isoform 1 and 5 show more tubular mitochondria compared to cells expressing only isoform 1, suggesting that additional production of s-OPA1 in this case promotes mitochondrial fusion (Fig. 2.5E). These results suggest that the ratio of 1-OPA1 to s-OPA1 is an important parameter affecting fusion levels.

At present, there is no evidence that the various short forms of OPA1 are functionally distinct with respect to their role in mitochondrial fusion. Our working model is that all the short forms are functionally similar. Mechanistically, the enhancement of tubular mitochondria by sOPA1 requires its GTP hydrolysis activity (Fig. S2.5C, E), suggesting that it plays an enzymatic role in this process. The multiple cleavage sites allow for production of short forms under basal and stress conditions.

Similar to these results, in an in vitro liposome fusion assay, 1-OPA1 was sufficient to mediate membrane fusion, but the addition of increasing amounts of s-OPA1 to the same reaction caused higher levels of liposome fusion (Ban et al., 2017b). A previous study suggested that 
overexpression of s-OPA1 leads to mitochondrial fission, a result interpreted as supporting a role in fission (Anand et al., 2014b). We cannot rule out a role of s-OPA1 in fission. However, it is possible that overexpression of s-OPA1 causes an imbalance of the 1- to s-OPA1 ratio, which we have shown is critical for controlling mitochondrial dynamics.

It should be noted that the S3 cleavage site we have discovered is a mechanism for constitutive cleavage in OPA1 isoforms containing exon $4 \mathrm{~b}$, rather than for induced cleavage under special physiological cues or cellular stress conditions, as is the case for S1 (constitutive and CCCPinducible). Unlike isoforms containing S1 and S2, which are partially cleaved to generate a cohort of long and short forms, isoforms containing S3 are fully processed under basal conditions to produce solely s-OPA1. The physiological importance of this constitutive cleavage is indicated by our results showing that removal of S3-containing isoforms can cause mitochondrial fragmentation under basal growth conditions.

\section{Materials and Methods}

\section{Antibodies}

The following antibodies were used: anti-Actin (Mab1501R, Millipore), anti-HSP60 (SC1054, Santa Cruz Biotech), anti-FLAG (F-1804, Sigma), anti-OPA1 (612606, BD Biosciences), anti-TOMM20 (FL-145, Santa Cruz Biotech), anti-TUBULIN (T6199, Sigma), anti-OMA1 (sc515788, Sigma), and anti-YME1L (11510, Protein Tech Group). 


\section{Western Blot}

Homemade $8 \%$ SDS-PAGE gel was used prior to switching to the high resolution gel system.

High resolution gel system: The Bio-Rad Mini-PROTEAN precast gel system was used for Western blotting. 10ug of each cell lysate sample was loaded onto 7.5\% Mini-PROTEAN TGX precast gels, run at $150 \mathrm{~V}$ at $4^{\circ} \mathrm{C}$ for 2 hours to resolve OPA1 bands, then transferred onto nitrocellulose membranes using Semi-dry Transfer Cell (Bio-Rad), immunoblotted with primary antibodies followed by horseradish peroxidase-conjugated secondary antibodies. Densitometry of Western blots was done using ImageJ.

\section{Plasmid construction and retroviral transduction}

The eight isoforms of human OPA1 cDNA were described previously (Song et al., 2007b). FLAG-tagged OPA1 isoforms were constructed by inserting a 3XFLAG sequence (amino acid sequence: DYKDHDGDYKDHDIDYKDDDDK) into the 3 ' end of the OPA1 gene at the MfeI/NotI site. OPA1 sequences are highly similar between mouse and human (Fig. S2.4B).

To generate the OPA1 bicistronic expression system, isoform 1 was cloned into pMSCVIRES-YFP II (Addgene, \#52108) at BamHI/ApaI sites. Isoform 5 was then cloned into NcoI/HpaI sites, replacing YFP.

To generate mutants of OPA1 isoform 5, primers containing deletion mutations were used to amplify 5' and 3' OPA1 fragments, which were then fused together by PCR. The amplified DNA fragments were subcloned back into the pMSCV-Opa1 isoform 5 vector and expressed via retroviral infection. 
To disrupt Opa1 exon 5b, CRISPR-Cas9 sgRNA was analyzed by the online gRNA design platform at crispr.mit.edu. The gRNA sequence with the highest score was selected and cloned into the pSpCas9n(BB)-2A-Puro plasmid. Transfection and selection were done as previously reported (Ran et al., 2013). The guide RNA was generated by the following oligonucleotides:

Forward: CACCGGGGCATAGCTCGTGCTATAC

Reverse: AAACGTATAGCACGAGCTATGCCCC

shRNAs were cloned into the BglII/EcoRI sites of the retroviral vector pRetroX-H1, which contains the $\mathrm{H} 1$ promoter. The shRNA target sequences were:

Opa1 exon4b: GGTTAGTGAAGTCCTAGAAGT

Oma1: GCAGTCTACCAGGATAAATCG

Yme11: GCAAATGCTCCTTGTGTTATA

Non-targeting: GACTAGAAGGCACAGAGGG

All constructs were authenticated by DNA sequencing.

\section{Mouse breeding}

Cas9 mRNA and sgRNA targeting Opa1 exon 5b were injected into fertilized oocytes at the one cell stage by the Transgenic/Knockout Rodent Core Facility at USC. The genotypes of the animals were analyzed by PCR and DNA sequencing, and animals with frameshift mutations were selected. Two homozygous mutant mouse lines - one with a 13-nucleotide deletion and the other with a 44-nt deletion-were found to lack OPA1 transcripts containing exon $5 \mathrm{~b}$. These $\Delta$ exon $5 \mathrm{~b}$ mice were subsequently crossed to Oma1-null mice to generate $\Delta$ exon 5b/Oma1-null animals. Mouse experiments were conducted under protocols approved by the Caltech Institutional Animal Care and Use Committee. 


\section{Cell lines and cell culture}

MEFs (mouse embryonic fibroblasts) were maintained in Dulbecco's Modified Eagle Medium (DMEM) supplemented with $10 \%$ fetal bovine serum (FBS) and $1 \%$ penicillin/streptomycin at $37{ }^{\circ} \mathrm{C}$ and $5 \% \mathrm{CO}$. The OXPHOS-inducing medium and glycolytic medium were described previously (Mishra et al., 2014b).

The Opa1-null, Oma1-null, Yme11-null, and Oma1/Yme11-null MEFs were described previously (Song et al., 2007b; Anand et al., 2014b). To generate $\triangle$ exon 5b MEFs, WT MEFs were transiently transfected with the CRISPR-Cas9 plasmid. 24 hours later the transfected MEFs were selected with $1 \mu \mathrm{M}$ puromycin for 24 hours, and cells were cloned by limiting dilution in 96-well plates. After expansion, the clones were lysed with RIPA buffer (ThermoFisher Scientific) and analyzed by Western blotting against OPA1. Clones with the expected OPA1 band pattern were expanded, and their genotypes were confirmed by DNA sequencing.

To generate $\Delta$ exon 5b/Oma1-null fibroblasts, cells were isolated from $\Delta$ exon $5 \mathrm{~b} / \mathrm{Oma} 1$ mouse tails at postnatal day 21 and grown in DMEM supplemented with $10 \%$ fetal bovine serum (FBS), essential amino acids, and penicillin/streptomycin at $37{ }^{\circ} \mathrm{C}$ and $5 \% \mathrm{CO}$. The fibroblasts were immortalized by expression of SV40 large T antigen. The genotype of the cells was confirmed by PCR and immunoblotting.

\section{Cell respiration measurement}

Oxygen consumption rate was measured in a Seahorse Biosciences Extracellular Flux Analyzer (model XF96), as described previously (Mishra et al., 2014b). 


\section{Imaging and quantification}

All images were acquired with a Zeiss LSM 710 confocal microscope with a PlanApochromat 63X/1.4 oil objective. Alexa 488 and Alexa 546 conjugated dyes were excited by the $488 \mathrm{~nm}$ laser and the $561 \mathrm{~nm}$ laser, respectively. Mitochondria were visualized and imaged by immunostaining with Tom20 antibody.

To determine mitochondrial fusion activity, cells were visually scored under a fluorescent microscope (Axiovert 200M; Carl Zeiss MicroImaging, Inc.) into four morphological classifications. Scoring was done manually, blinded. "Fragmented" refers to cells that contain spherical mitochondrial fragments with no more than two short tubules found. "Short tubular" refers to cells with a mixture of fragmented and short tubular mitochondria. "Long tubular" refers to cells in which mitochondria were elongated, but not highly interconnected into a mitochondrial mesh. "Interconnected" refers to cells in which mitochondria formed a highly interconnected network of mitochondrial filaments, with few isolated mitochondria. All data are presented as mean \pm s.d (standard deviation) unless otherwise noted. Quantitative data were analyzed by Student's t-test for the comparison between two groups (two tailed, unpaired).

\section{PA-GFP fusion assay}

Cells expressing matrix DsRed and photoactivatable GFP (PA-GFP) targeted to either the $\mathrm{OM}$ or matrix were plated on glass coverslips and imaged live at $37^{\circ} \mathrm{C}$ on an LSM 710 confocal microscope (Carl Zeiss, Inc.). Measurements were initiated 1-2 hours after medium change. PAGFP was photoactivated in a region of interest $(5 \mu \mathrm{m} \times 5 \mu \mathrm{m})$ by illumination with a $405 \mathrm{~nm}$ laser. The activated fluorescent signal was then collected (for the entire cell) every 3 min for the next 
30 min using a spectral detector, followed by spectral unmixing based on the individual PA-GFP and DsRed spectra. Fusion events result in the dilution of the activated signal. The average pixel intensity (for the entire cell) over time is a measurement of fusion rates (Karbowski et al., 2014). Images were analyzed in MatLab (Mathworks, Inc.). P values were calculated using a Student's t test on the slopes of intensity versus time for individual measurements ( $>20$ per condition).

\section{Mass Spectrometry}

At 48 hours after transient transfection of FLAG-tagged isoform 5 in 293T cells, cell lysates were harvested and immune-precipitated against FLAG. Concentrated samples were run on SDS-PAGE, and colloidal Coomassie blue stain (Invitrogen) was used to identify the bands. The desired bands were excised, finely diced, and transferred to a microcentrifuge tube. The gel pieces were reduced, alkylated, and digested by trypsin overnight at $37^{\circ} \mathrm{C}$. Digested peptides were extracted from the gel, lyophilized, and desalted by C18 ZipTips (EMD Millipore). The peptides were separated with a 60 min gradient at a flow rate of $220 \mathrm{~nL} / \mathrm{min}$ using a nanoflow LC system, EASY-nLC 1000, (Thermo Fisher Scientific). The separated peptides were ionized by a Nanospray Flex ion source and subjected to MS/MS analysis using a Fusion Orbitrap mass spectrometer (Thermo Fisher Scientific, San Jose). LC-MS/MS Data Analysis: The Thermo raw data file was converted to mzML file using MSconvert. The mzML file was searched using MS-GF+ against the Opa1 sequence. Default options were used except precursor mass tolerance was set to 7 ppm, instrument was specified to "Orbitrap/FTICR/Lumos" (1), and the enzyme was "Unspecific cleavage" (0). Results were further refined by filtering out all peptide-spectrum matches with an E-value greater than 0.001 . 
Acknowledgements: We thank Thomas Langer (Max-Planck-Institute for Biology of Ageing) for the Oma1-null, Yme11-null, and Oma1/Yme11-null cells. We thank Carlos López Otin (Universidad de Oviedo) for the Oma1 knockout mice.

Conflict of interest: The authors declare they have no conflicts of interest with the contents of this article.

Author contributions: RW and DCC conceptualized this project, analyzed the data, and wrote the manuscript. RW carried the experimental analysis. PM performed the PA-GFP fusion assays. AM performed the LC-MS experiments, and the data were analyzed by SDG and MJS. 


\section{Figure Legends}

Figure 2.1: OMA1 and YME1L differentially regulate OPA1 fusion activity.

(A) Schematic of mouse OPA1 protein isoforms, showing the origin of protein bands a-e. MEFs express isoforms $1,5,7$, and 8 . Isoforms 1 and 7 produce long forms that constitute bands a-b and short forms that constitute bands c-e. Isoforms 5 and 8 produce exclusively short forms expected to co-migrate with bands c-e, but for simplicity, bands c-e are labeled according to their origin from isoforms 1 and 7. The MPP (Mitochondrial Processing Peptidase), S1 (by OMA1), and S2 (by YME1L) cleavage sites are indicated with arrows. Orange line and arrows show that band c and $\mathrm{d}$ are derived from long isoform 7/a, pink line and arrow show that band e is derived from the long isoform 1/b. (B) Western blot analysis of OPA1 bands in Oma1 and Yme11 mutant MEFs. Five OPA1 bands (a-e) are apparent in wildtype MEFs. Oma1-null MEFs lack c and e; Yme11null MEFs lack d; and Oma1/Yme11-null MEFs lack all the short forms, c-e. Tubulin was used as loading control. (C) Representative images of mitochondrial morphology (mito-DsRed) in WT and protease-null MEFs in different media. GLY: high glucose medium; OXI: OXPHOS-inducing medium; CHX: high glucose medium with $10 \square \mathrm{M}$ cycloheximide. Insets show magnified view. Scale bar, $5 \mu \mathrm{m}$. (D) Quantification of mitochondrial morphology of cells in (C). In each experiment, 100 cells were scored. Error bars show standard deviation from three independent experiments. (E) Comparison of mitochondrial fusion rates in vivo in different media between WT and Oma1/Yme1l-null MEFs. Fusion activity was measured by the intensity reduction of PA-GFP as a function of time. Error bars represent standard deviations from at least 6 independent measurements. 
Figure 2.2: The S2 site encoded by Opa1 exon 5b is necessary for OXPHOS-induced fusion in vivo.

(A) Schematic of exon 5b, showing locations of S2 and CRISPR-Cas9 gRNA target. Deletions present in clones 1 and 2 are indicated. (B) Schematic of OPA1 isoform composition after exon 5b-containing isoforms are eliminated; Red X's indicate isoforms expected to be missing in mutant cells. (C) Western blot analysis of $\triangle$ exon 5b MEF clones. Clones 1 and 2 are positive clones that show the expected disappearance of bands a, c, and $\mathrm{d}$. The last lane is a negative clone. Tubulin was used as loading control. (D) Quantification of mitochondrial morphology in WT and two $\Delta$ exon $5 \mathrm{~b}$ clones in the indicated media. 100 cells were counted for each experiment. Error bars indicate standard deviation from three independent experiments.

\section{Figure 2.3: Detection of a new OPA1 cleavage in WT and $\Delta$ exon $5 b$ cells.}

(A) Higher resolution Western analysis of WT, $\Delta$ exon 5b, and $\triangle$ exon 5b/Oma1-null MEFs. Note the additional band located under d in WT MEFs. This band (referred to as d') is more clearly seen in $\Delta$ exon $5 \mathrm{~b}$ and $\Delta$ exon 5b/Oma1-null clones. HSP60 was used as loading control. (B) Dependence of band d' on Yme1l. Cells expressing the indicated shRNA were analyzed by Western blotting. The two sets of panels were run on the same gel. HSP60 was used as loading control. (C) PCR analysis of Opa1 transcripts in $\Delta$ exon $5 \mathrm{~b}$ cells. The forward primer is located in exon 3 , and the reverse primer is located in exon 7 (Fig. S3C). Bands corresponding to isoforms 1 and 5 were confirmed by sequencing. (D) Schematic of full-length OPA1 isoforms 1 and 5. (E) Western blot analysis of the short form produced by isoform 5; HSP60 was used as loading control. (F) Dependence of isoform 5 band d' on YME1L. FLAG-tagged human OPA1 (isoform 1 or 5) was 
expressed in cells of the indicated genotype and analyzed by Western blotting against FLAG; HSP60 was used as loading control.

\section{Figure 2.4: Identification of the YME1L-dependent OPA1 S3 cleavage site within exon 4b.}

(A) Dependence of band d' on exon 4b. ShRNA against Opa1 exon $4 \mathrm{~b}$ was expressed in cells of the indicated genotype. Asterisks highlight band d'. HSP60 was used as loading control. (B) Schematic of Opa1 exon $4 \mathrm{~b}$ mutants. On top, the polypeptide sequence encoded by exon $4 \mathrm{~b}$ (red) and exon 5 (blue) is shown. The red arrow indicates the known S1 cleavage site in exon 5; the green arrows indicate the new S3 sites found in this study. The isoform 5 mutants are listed, along with amino acids deleted within exon $4 \mathrm{~b}$. (C) Effect of exon $4 \mathrm{~b}$ mutants on OPA1 processing. FLAG-tagged exon $4 \mathrm{~b}$ mutants of human isoform 5 were expressed in Opa1-null MEF cells and analyzed by Western blot. FLAG-tagged WT isoforms 1 and 5 were used as controls. HSP60 was used as loading control. (D) Effect of OMA1 and YME1L on OPA1 processing in isoform 5 mutants. WT human Iso 5 and its mutants 2, 6 and 7 were expressed in Oma1- and Yme11- null MEFs; FLAG-tagged OPA1 band patterns were analyzed by Western blot. HSP60 was used as loading control. (E) Identification of likely S3 cleavage sites within exon $4 \mathrm{~b}$ by tandem mass spectrometry of band d'. Band d' was produced by expression of FLAG-tagged Opa1 isoform 5 in $293 \mathrm{~T}$ cells, purified by anti-FLAG immunoprecipitation, resolved by SDS-PAGE, digested by trypsin, and subjected to tandem mass spectrometry. Left: Schematic indicates the three ragged Nterminal peptides identified, colored according to their relative intensity. Right: Plot of the intensity of the three peptides. (F) Effect of penta-leucine mutants on generation of band d'. As indicated, alanines were used to replace 1, 2, or 3 leucines within the C-terminal penta-leucine stretch encoded by exon 4b. FLAG-tagged mutants of isoform 5 were expressed in Opa1-null cells 
and analyzed by Western blot. HSP60 was used as loading control. (G) Schematic of the OPA1 isoform profile in $\triangle$ exon 5b MEFs. Green arrow represents the new S3 site identified.

\section{Figure 2.5: The S3-cleaved short form of isoform 5 regulates mitochondrial morphology.}

(A) Elongation of mitochondria by isoform 5 mutants. Isoform 5 mutants were expressed in Opa1 null cells, and mitochondrial morphologies were quantified. Error bars indicate standard deviation from six independent experiments. (B) Depletion of band d' by shRNA against exon $4 \mathrm{~b}$ and reexpression of d' by isoform 5. Ectopic expression of isoform 5 is sufficient to overcome the shRNA effect and produce d'. OPA1 isoforms were analyzed by Western blotting against OPA1; HSP 60 was used as loading control. (C) Effect of exon $4 \mathrm{~b}$ shRNA on mitochondrial morphology. Error bars indicate standard deviation from three independent experiments. (D) Analysis of bicistronic OPA1 expression. Opa1-null cells expressing isoform 1 or isoform 1 plus isoform 5 were analyzed by Western blotting against OPA1. $\Delta$ exon 5 b cells are shown for comparison. HSP60 was used as loading control. (E) Effect of band d' on mitochondrial morphology. Quantification of mitochondrial morphology of Opa1-null MEFs expressing isoform 1 with YFP or isoform 1 with isoform 5. Error bars indicate standard deviation from three independent experiments. $\mathrm{P}$ values for the fragmented, short tubular, long tubular, and interconnected were 4.8x10-4, 0.04, 0.001, 9.9x107, respectively. P-values were calculated by Student's t-test. (F) Model showing the OPA1 isoforms under normal and S3 disrupted conditions. When S3 is disrupted (bottom panel, box), cleavages at S1 and cryptic sites are activated, preserving production of s-OPA1. OM: outer membrane, IM: inner membrane. 
A

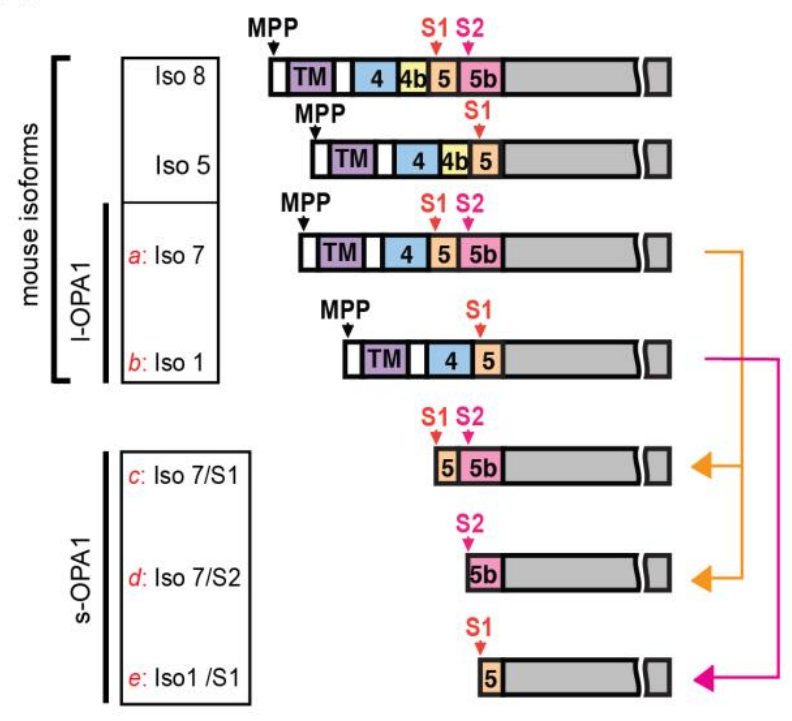

B

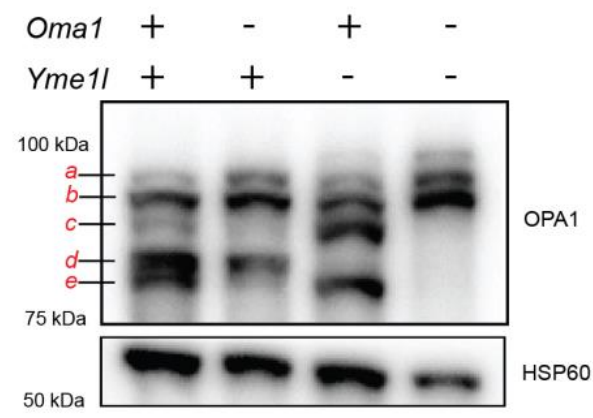

D

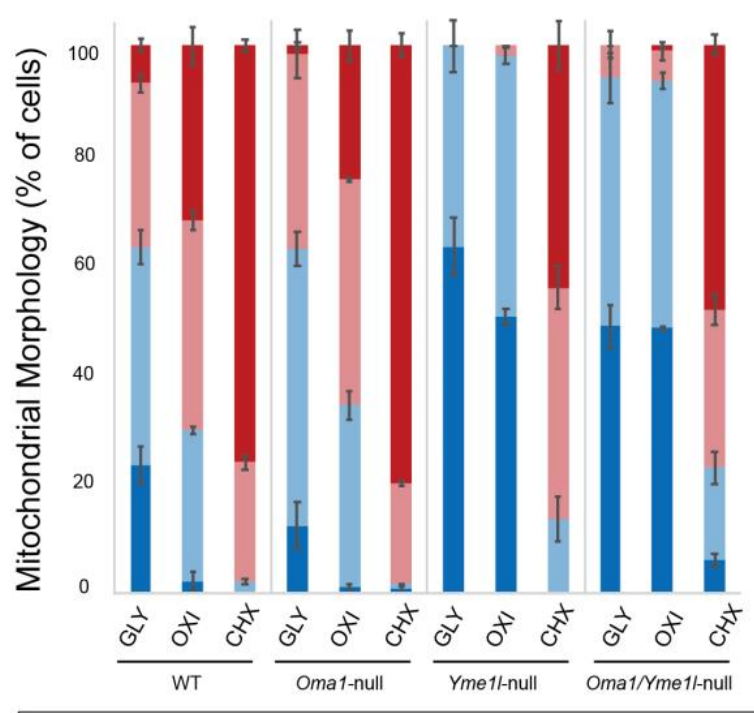

Interconnected Long Tubular $\square$ Short Tubular $\square$ Fragmented
C

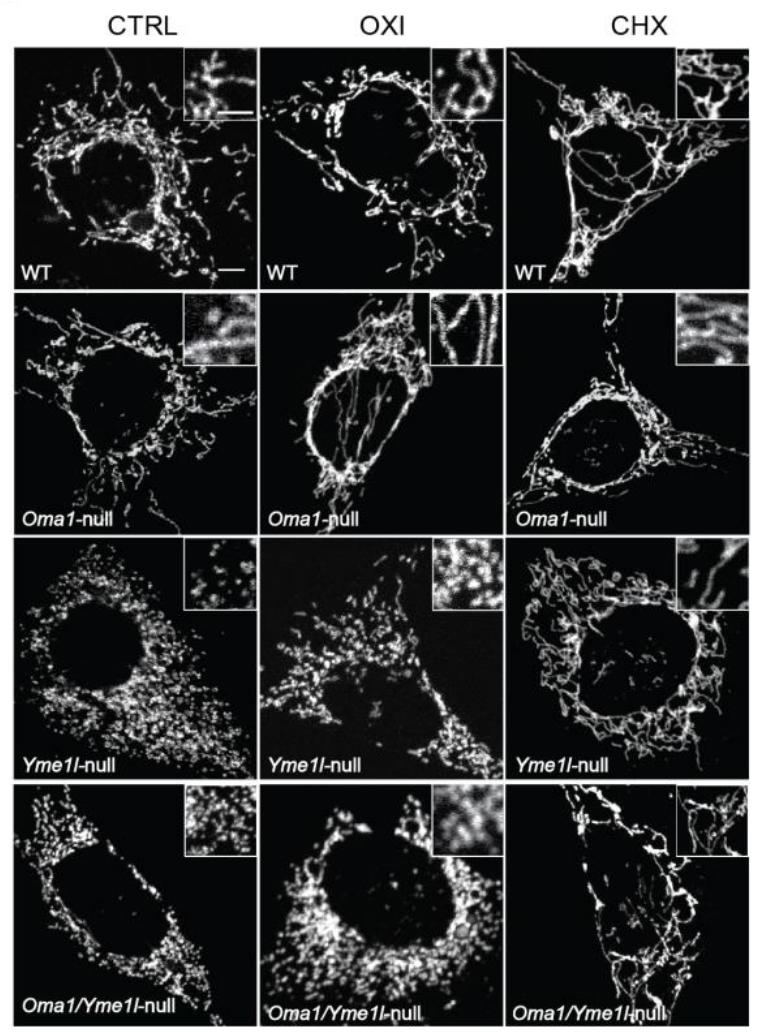

E

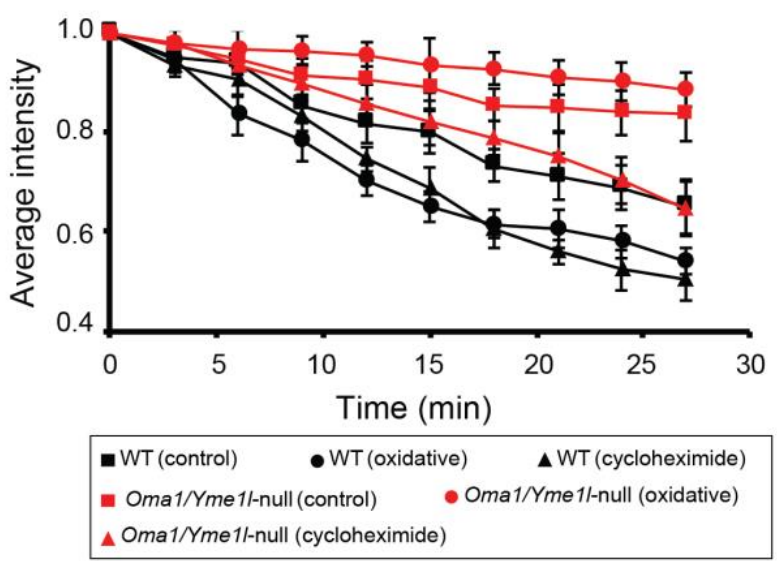

Figure 2.1: OMA1 and YME1L differentially regulate OPA1 fusion activity. 
A

GGTCTGCTTGGTGAGCTCATTCTCTTACAACAGCAAATTCAAGAGCACGAAGAGGAAGCACGCAGAGCCGCTGGCCAGTA--GCACGAGCACGAGCTATGCCCAACAGAAGCGCAAGG

Clone 1 GGTCTGCTTGGTGAGCTCATTCTCTTACAACAGCAAATTCAAGAGCACGAAGAGGAAGCACGCAGAGCCGCTGGCCAGTA--GCACGAGCACGAGCTATGCCCAACAGAAGCGCAAGG

GGTCTGCTTGGTGAGCTCATTCTCTTACAACAGCAAATTCAAGAGCACGAAGAGGAGGCACGCAGAGC

GGTCTGCTTGGTGAGCTCATTCTCTTACAACAGC---TATGCCCAACAGAAGCGCAAGG

B
a: Iso $7 \quad$ TTM
MPP
MPP $\quad$ S1 S2
b: Iso 1

\begin{tabular}{l|l|l|l|} 
MPP & \multicolumn{3}{c}{$s 1$} \\
\hline \\
\hline TTM & 4 & 5 & \\
\hline
\end{tabular}
$\sqrt{ }$
S1 S2
C: Iso $7 / S 1$
$55 b$
s2
d: Iso $7 / S 2$
$5 b$

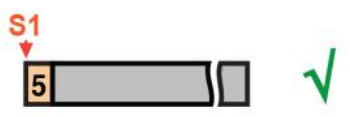

C

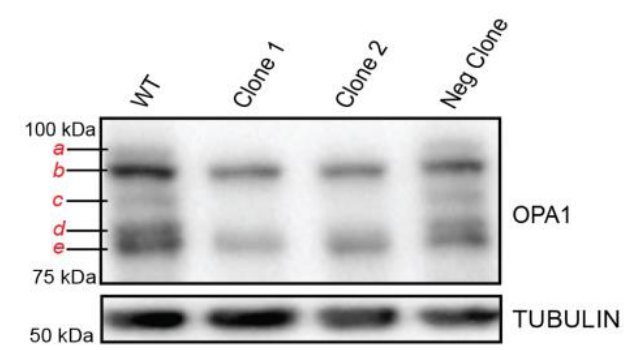

$\Delta$ exon $5 b$

D

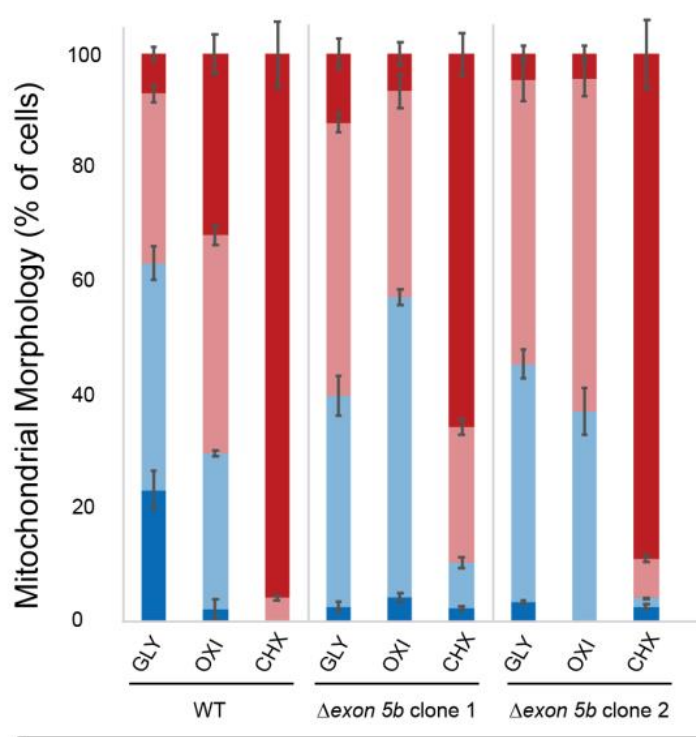

- Interconnected $\mathbf{n}$ Long Tubular $\mathbf{n}$ Short Tubular a Fragmented

Figure 2.2: The S2 site encoded by Opa1 exon 5b is necessary for OXPHOS-induced fusion in vivo. 
A

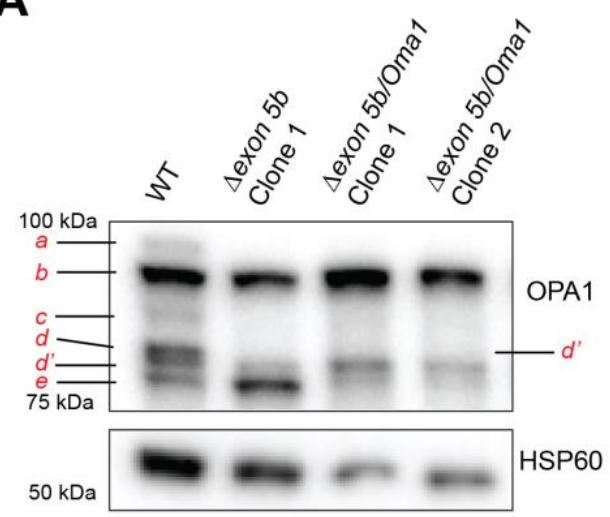

B

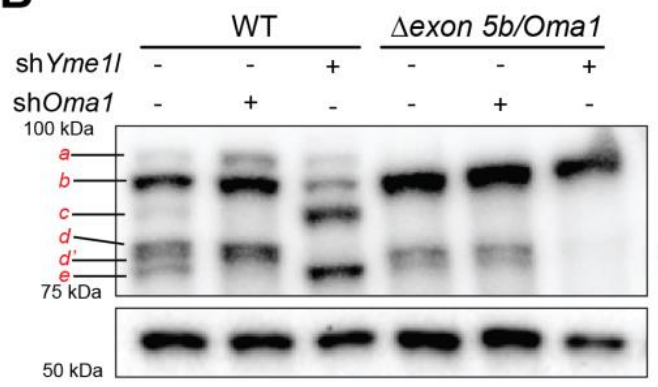

C

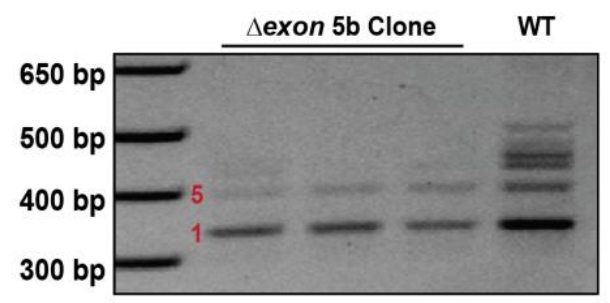

D

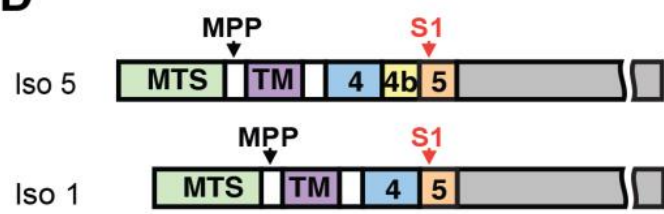

$\mathbf{E}$

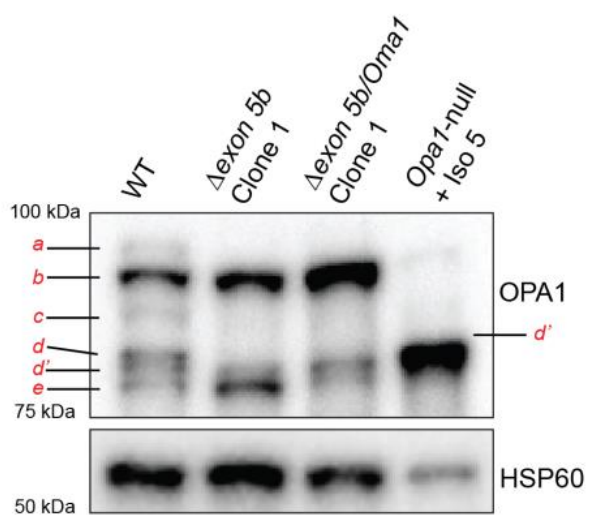

$\mathbf{F}$

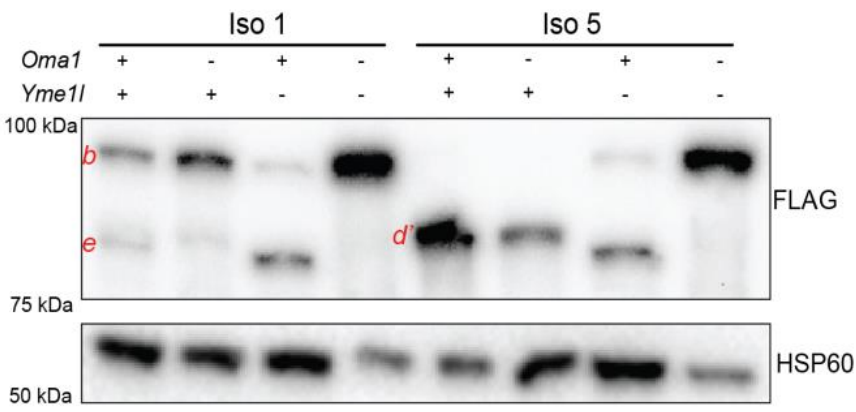

Figure 2.3: Detection of a new OPA1 cleavage in WT and $\Delta$ exon $5 \mathrm{~b}$ cells. 
A

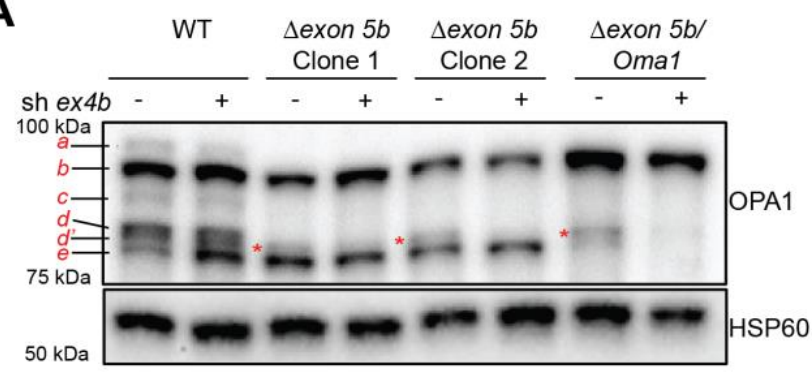

B

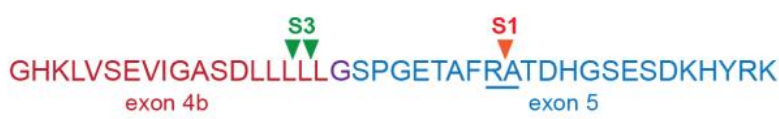

Mut 1: $\triangle \mathrm{HKLV}$

Mut 2: $\triangle$ VIGA

Mut 3: $\triangle$ SEVI

Mut 4: $\triangle I G A S D$

Mut 5: $\triangle \mathrm{GAS}$

Mut 6: $\triangle$ ASDL

Mut 7: $\triangle$ GASDLLLLL

Mut 8: $\Delta \mathrm{LLLL}$

C

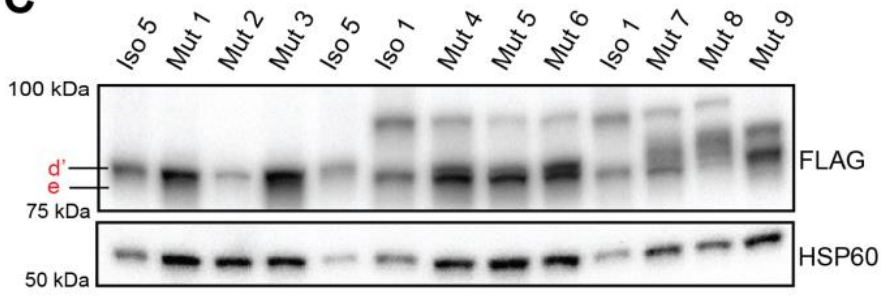

D

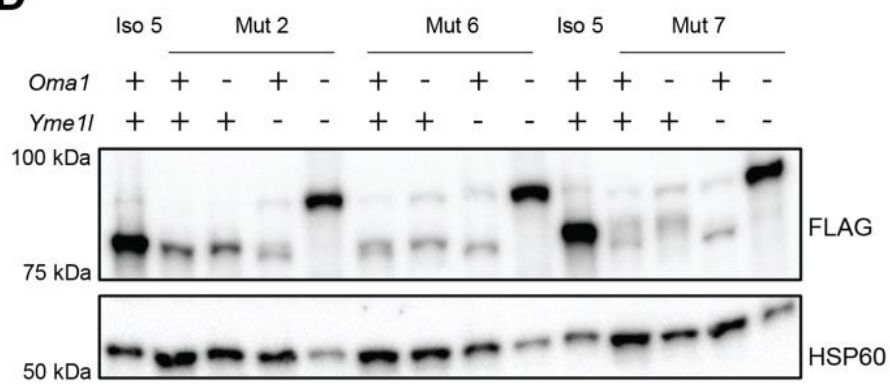

$\mathbf{F}$

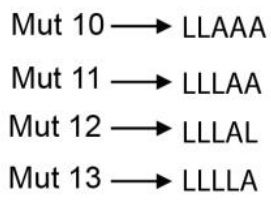

Mut $10 \longrightarrow$ LLAAA

Mut $12 \longrightarrow$ LLLAL

Mut $13 \longrightarrow$ LLLLA

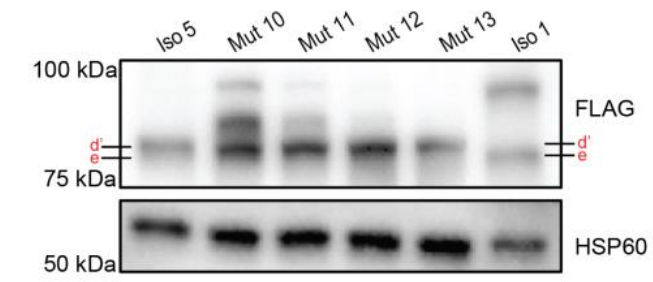

G

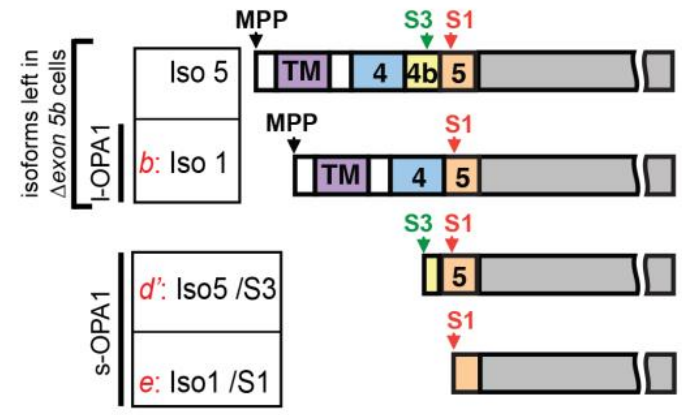

E
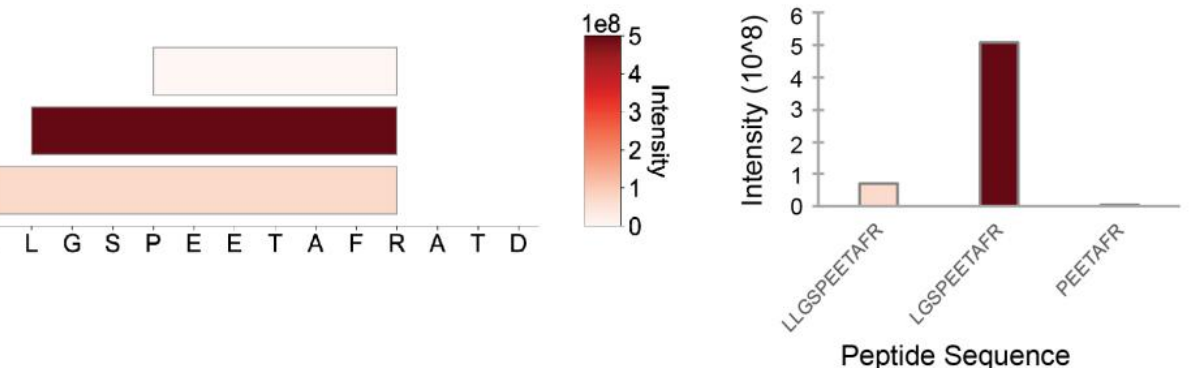

Figure 2.4: Identification of the YME1L-dependent OPA1 S3 cleavage site within exon 4b. 
A

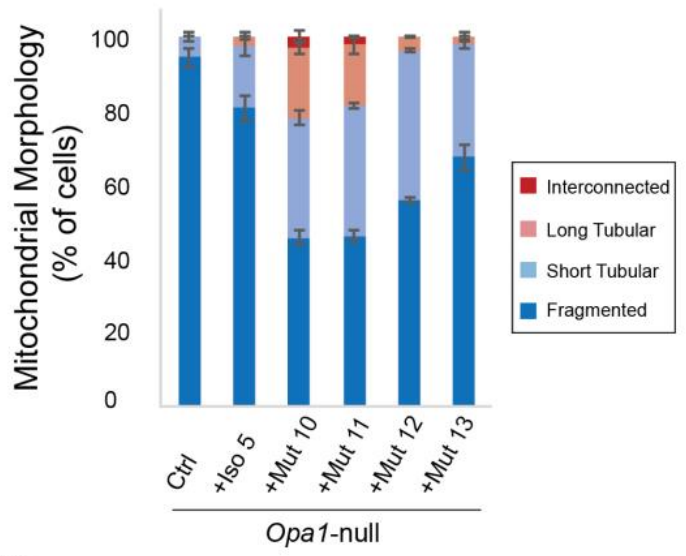

B

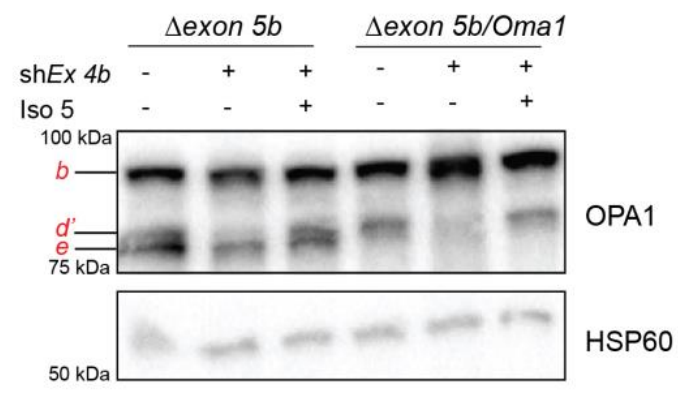

C

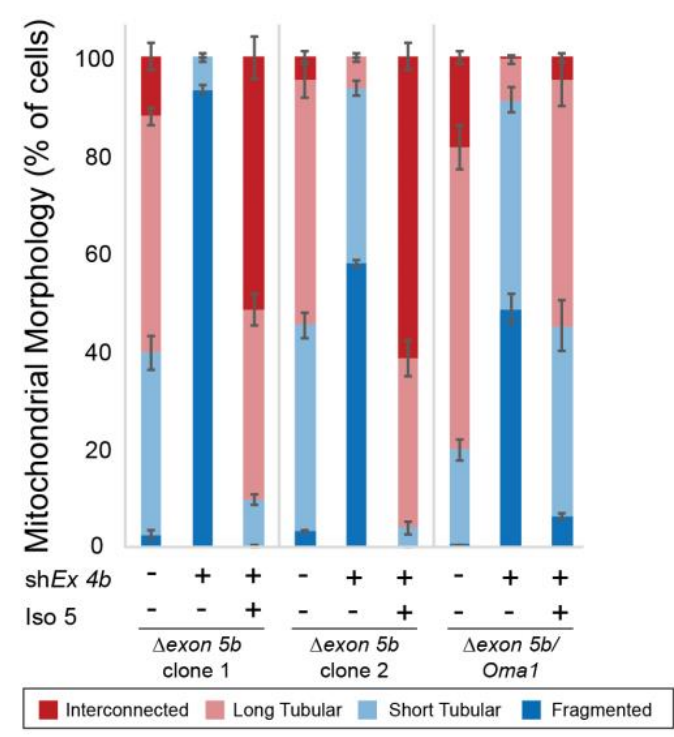

D

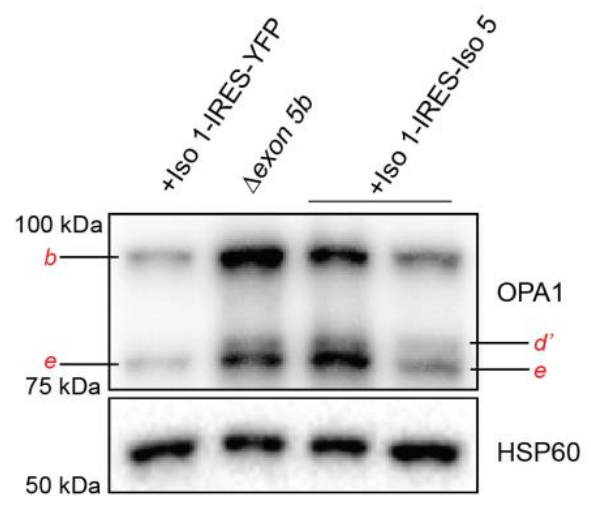

E

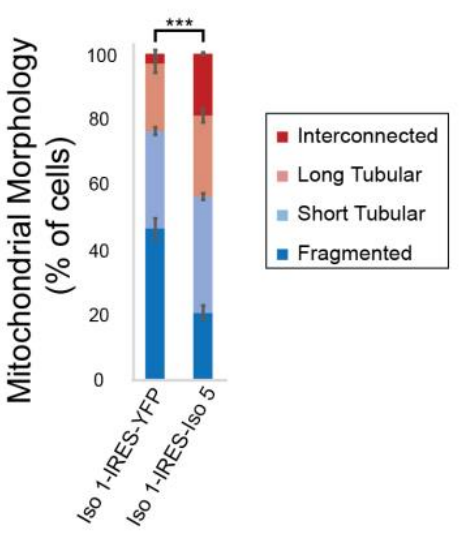

$\mathbf{F}$
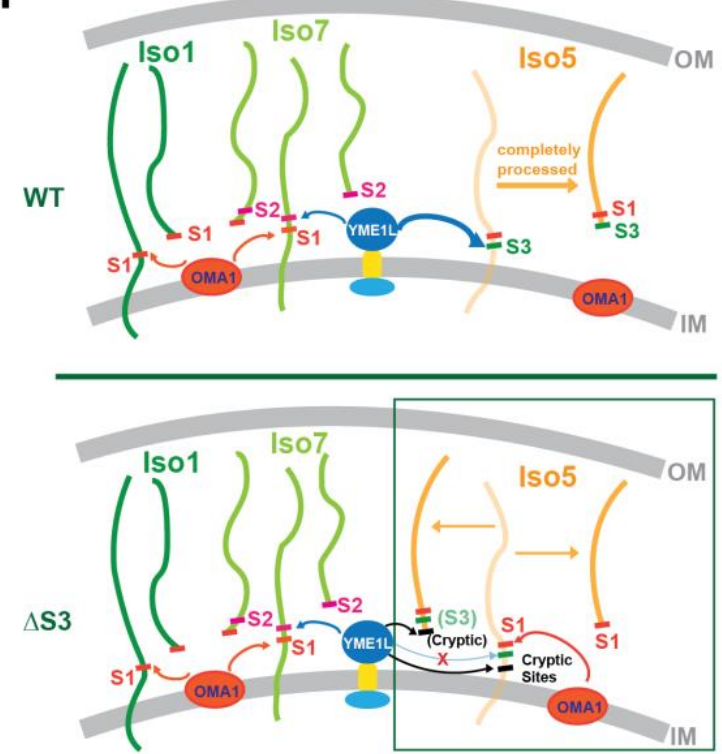

Figure 2.5: The S3-cleaved short form of isoform 5 regulates mitochondrial morphology. 


\section{Figure S2.1: OPA1 mRNA isoforms and the role of Oma1.}

(A) Schematic of the eight human OPAl isoforms. The isoforms differ based on alternative splicing of exons $4,4 \mathrm{~b}$, and 5b, and are grouped based on the production of 1-OPA1 or s-OPA1. The sites of the S1 and S2 cleavage sites are indicated. MPP: mitochondrial processing peptidase. (B) Tabulation of the isoforms, band pattern and mitochondrial morphology in wildtype and protease mutant MEF lines. (C) Verification of protease mutant cell lines by Western blotting. OMA1 and YME1L Western blots show the absence of corresponding protease in each cell line. Tubulin was used as loading control. (D) OPA1 band pattern in protease mutant cells grown in different media conditions. GLY: high glucose medium; OXI: OXPHOS-inducing medium; CHX: high glucose medium with $10 \mu \mathrm{M}$ cycloheximide.

\section{Figure S2.2: Phenotypic characterization of $\triangle e x o n 5 b$ MEFs and mice.}

(A) Representative images of mitochondrial morphology (Tom20 immunostaining) in WT and two $\triangle$ exon $5 b$ MEF clones in different media (supplemental data for Fig. 2D). GLY: high glucose medium; OXI: OXPHOS-inducing medium; CHX: high glucose medium with $10 \mu \mathrm{M}$ cycloheximide. Scale bar, $5 \mu \mathrm{m}$. (B) Seahorse data on cell respiration rate change after media switch from control glycolytic medium to OXPHOS-inducing medium. The blue arrow represents the time of media change. (C) Cell numbers in control glycolytic medium versus OXPHOS-inducing medium. Two sets of cells were seeded and grown in high glucose DMEM medium for 24 hours, washed with PBS, and transferred to control glycolytic medium or OXPHOS-inducing medium. Error bar: Standard deviation of three counts from the same experiment. (D) Western blot analysis of OPA1 in tissue lysates from WT and two $\Delta$ exon $5 b$ mutant mice. The disappearance of band $c$ was the most obvious change. Mut 1: mutant mouse 
line with 13 nucleotides deletion in Opal exon 5b; Mut 2: mutant mouse line with 44 nucleotides deletion in Opal exon 5b. (E) Weight of $\Delta$ exon $5 b$ (Mut 2) animals at week 24. Error bars indicate standard deviation; $n=6$ for each group.

\section{Figure S2.3: shRNA knockdown efficiency and primer design for Opa1 mRNA identification.}

(A) Efficiency of Omal knockdown analyzed by western blotting. shRNA-1 was used in the study. (B) Efficiency of Yme1L knockdown analyzed by western blotting. shRNA-1 was used in the study. Actin was used as the loading control. (C) Primers used for analysis of mRNA transcripts. Green arrowheads indicate the primers. The forward primer $(F)$ is in exon 3, and the reverse primer $(\mathrm{R})$ is in exon 7. Expected sizes for each amplified fragment are listed.

Figure S2.4: Tubulation of mitochondria by isoform 5 mutants.

(A) Quantification of mitochondrial morphology of Opal-null MEFs expressing WT or mutant FLAG-tagged isoform 5 (mutant 3-9). 100 cells were counted for each experiment. Error bars: standard deviation from three independent experiments. (B) Genomic and amino acid sequence comparison between mouse Opal and human OPAl exon 4b. Bold letters represent conserved sequences between mouse and human, and red letters indicate the exon 4b shRNA-targeted sequence. In the amino acid sequence, amino acids in parentheses are encoded partially by the adjacent exon.

Figure S2.5: Short form of isoform 5 regulates mitochondrial morphology. 
(A) Representative images of mitochondrial morphology (mt-Dsred) in Opal-null MEFs expressing isoform 5 and its mutant 10-13 (supplemental for Fig. 5A). Scale bar, $5 \mu \mathrm{m}$. (B) Representative images of mitochondrial morphology (Tom20 immunostaining) in cells shown in Fig. 5B. Scale bar, $5 \mu \mathrm{m}$. (C) Representative images of mitochondrial morphology (Tom20 immunostaining) in Opal-null MEFs expressing Iso1-IRES-Iso5 or Iso1-IRES-Iso-5 G300E. Scale bar, $5 \mu \mathrm{m}$. (D) Western blot against OPA1 to confirm the expression of Iso 1-IRES-Iso5 G300E. HSP60 was used as loading control. (E) Quantification of mitochondrial morphology in Opal-null MEFs expressing Iso 1, Iso1-IRES-Iso5, or Iso1-IRES-Iso5 G300E. Error bars indicate standard deviation from three independent experiments. 


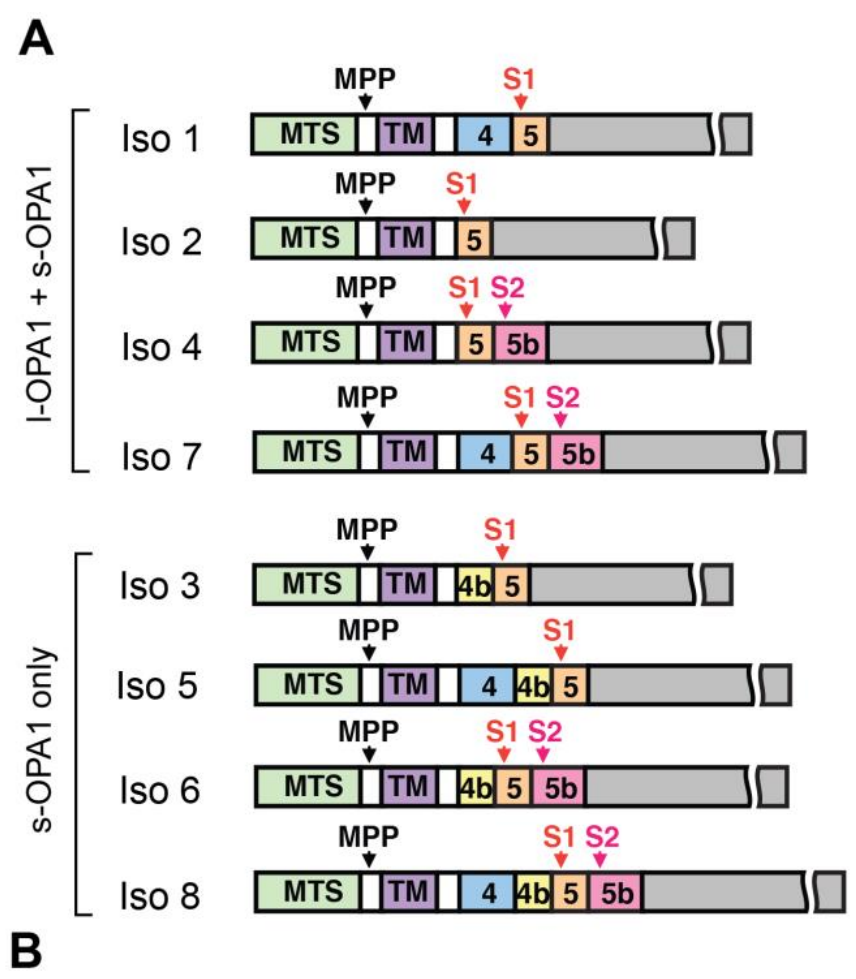

\begin{tabular}{|c|c|c|c|}
\hline Cell Line & Opa1 Isoforms & Band Pattern & $\begin{array}{c}\text { Mitochondrial } \\
\text { Morphology }\end{array}$ \\
\hline WT & $\begin{array}{c}\text { Iso 7L, Iso 1L } \\
\text { Iso 7/S1, Iso 7/S2, Iso 1/S1 }\end{array}$ & $\begin{array}{c}a, b \\
c, d, e\end{array}$ & Normal \\
\hline Oma1-null & $\begin{array}{c}\text { Iso 7L, Iso } 1 \mathrm{~L} \\
\text { Iso 7/S2 }\end{array}$ & $\begin{array}{c}a, b \\
d\end{array}$ & Normal \\
\hline Yme1L-null & $\begin{array}{c}\text { Iso 7L, Iso } 1 \mathrm{~L} \\
\text { Iso 7/S1, Iso 1/S1 }\end{array}$ & $\begin{array}{c}a, b \\
c, e\end{array}$ & Fragmented \\
\hline Oma1/Yme1L-null & Iso 7L, Iso 1L & $a, b$ & Partially Fragmented \\
\hline
\end{tabular}

C
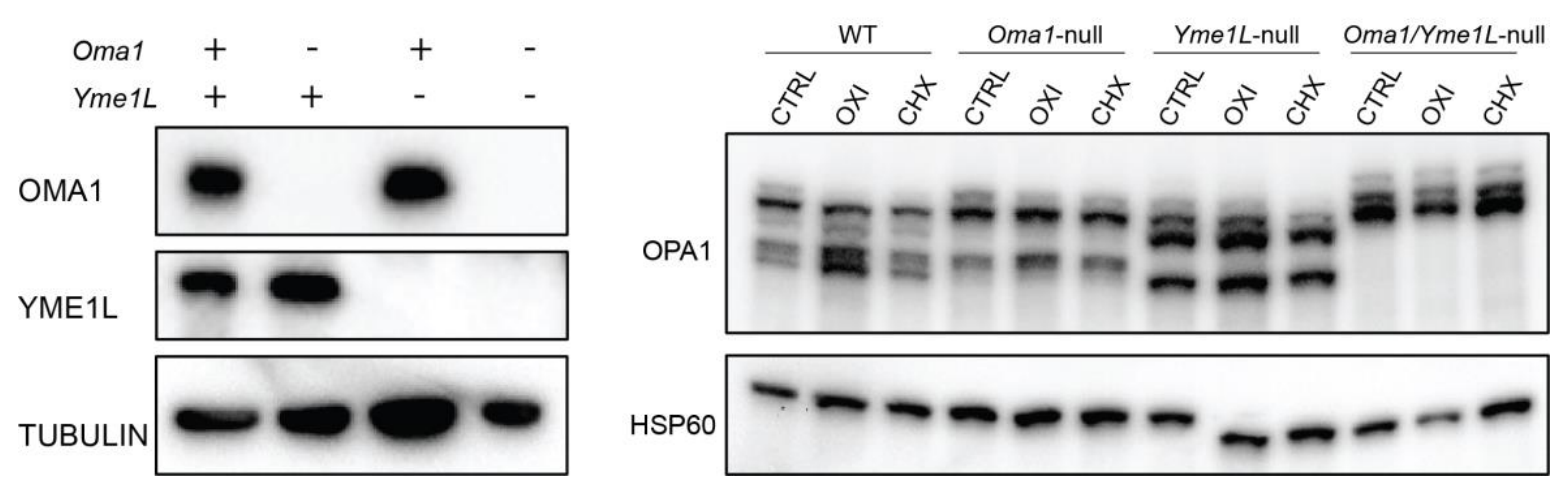

Figure S2.1: OPA1 mRNA isoforms and the role of Oma1. 
A
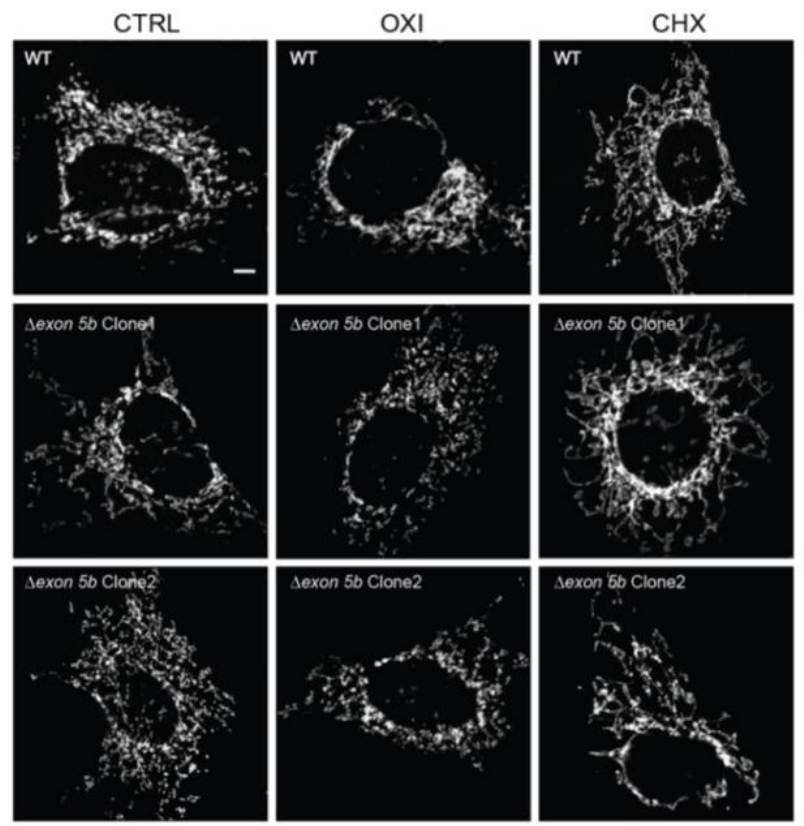

B
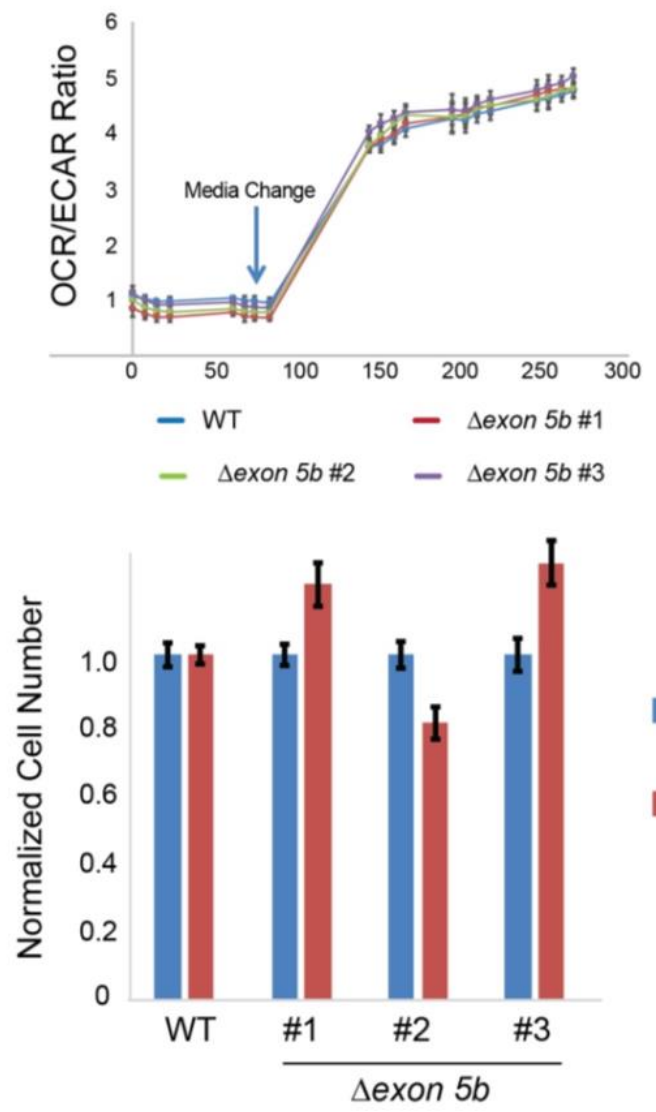

D
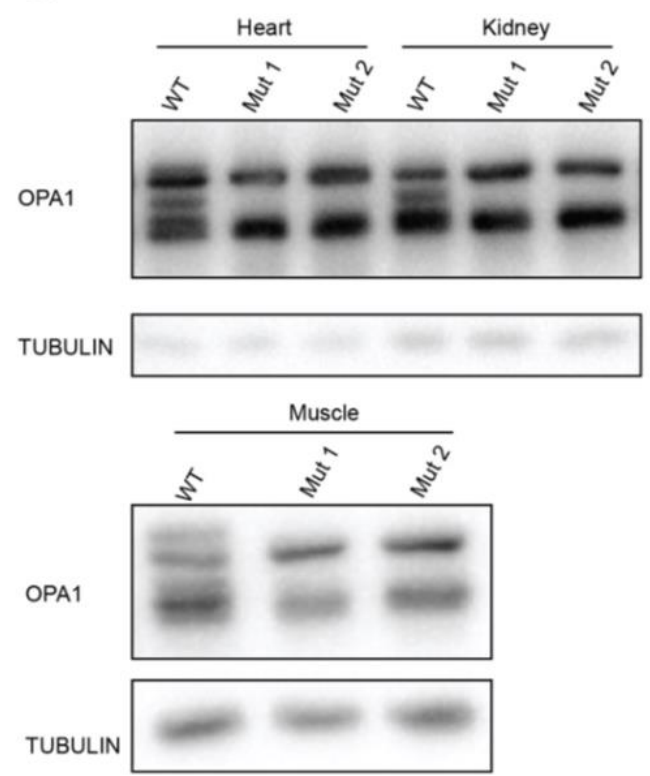

E

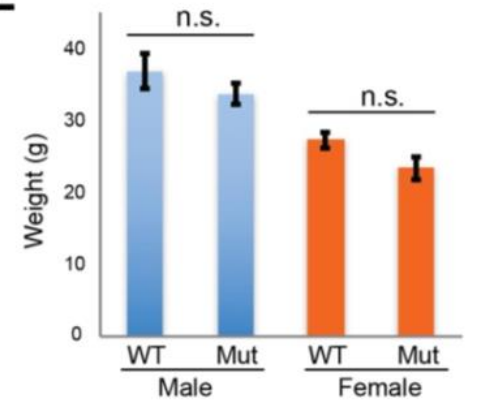

Figure S2.2: Phenotypic characterization of $\triangle e x o n 5 b$ MEFs and mice. 
A
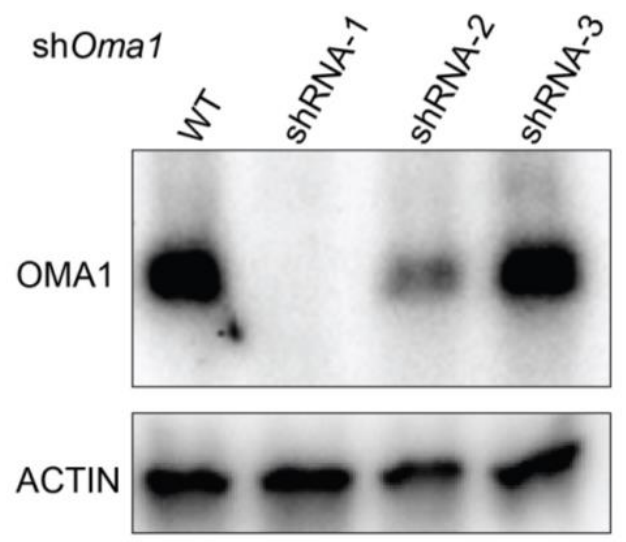

B

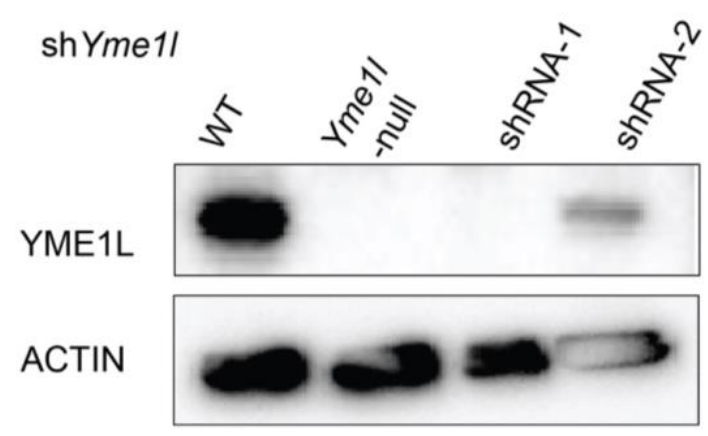

C

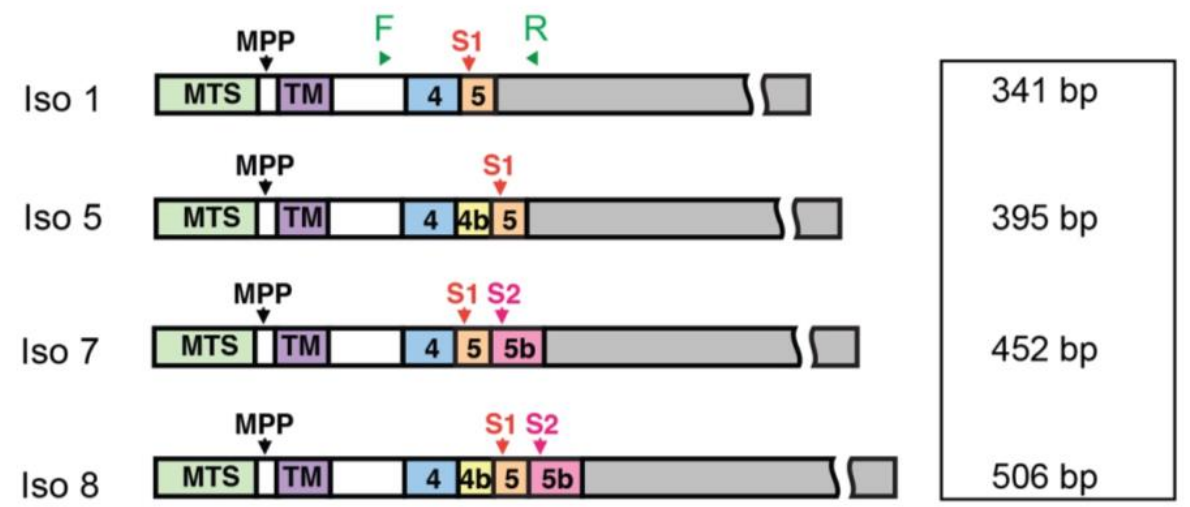

Figure S2.3: shRNA knockdown efficiency and primer design for Opal mRNA identification. 
A

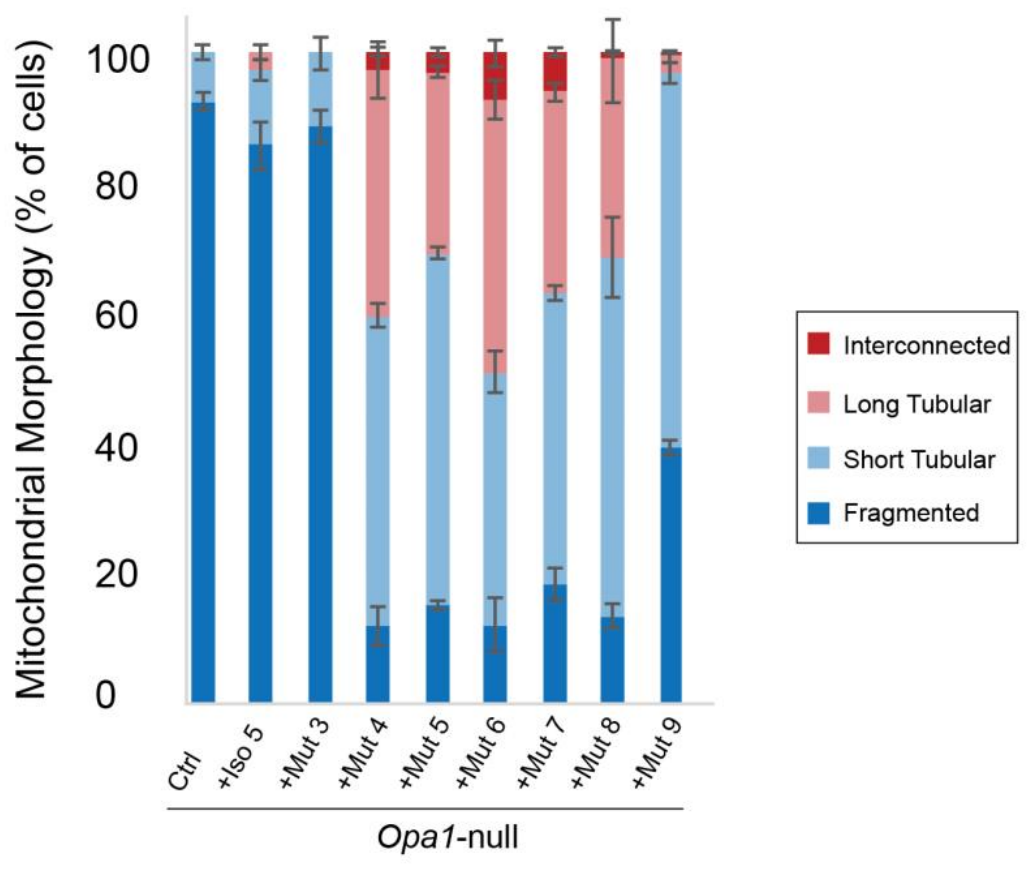

Genomic Sequence:

mouse: GTCCCAAATTGGTTAGTGAAGTCCTAGAAGTTTCTGAGGCCCTTCTCTTGTTAG

human: CACAAATTGGTAGTGAAGTCATAGGAGCTTCTGACCTACTTCTCTTGTTAGGT

Amino Acid Sequence:

mouse: (G) PKLVSEVLEVSEALLLL ( G)

human: ( G ) HKLVSEVIGASDLLLLL ( G)

Figure S2.4: Tubulation of mitochondria by isoform 5 mutants. 
A
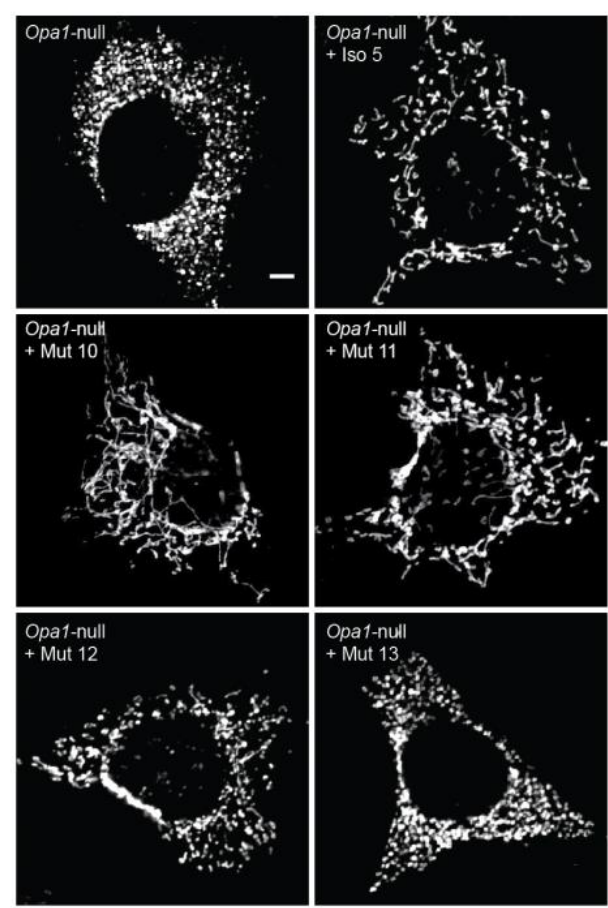

B
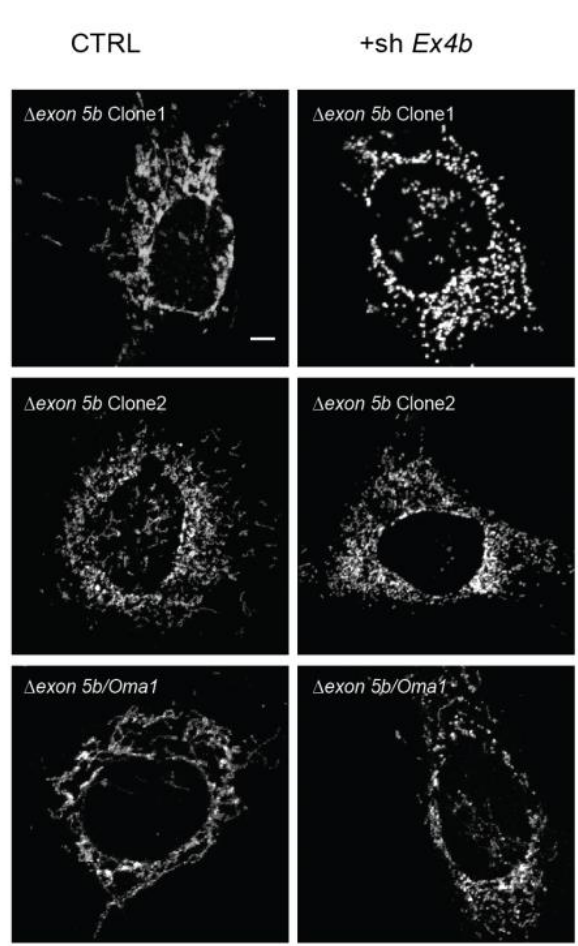
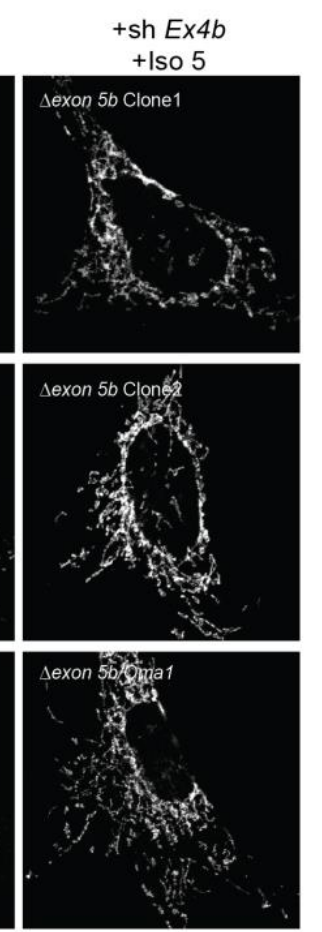

$\mathbf{E}$

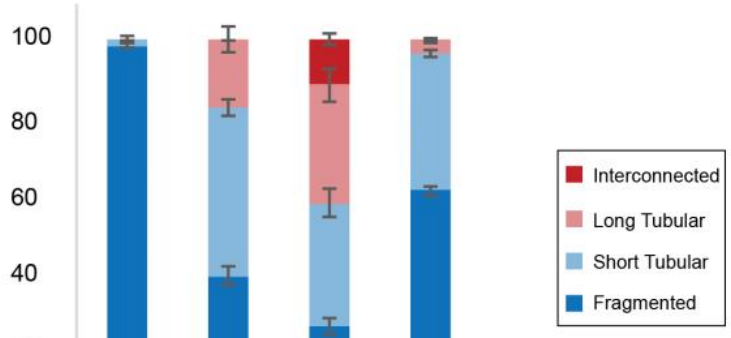

Figure S2.5: Short form of isoform 5 regulates mitochondrial morphology. 


\section{References}

Akepati, V.R., E.-C. Müller, A. Otto, H.M. Strauss, M. Portwich, and C. Alexander. 2008. Characterization of OPA1 isoforms isolated from mouse tissues. J. of Neurochemistry. 106:372-383. doi:10.1111/j.1471-4159.2008.05401.x.

Alavi, M.V., S. Bette, S. Schimpf, F. Schuettauf, U. Schraermeyer, H.F. Wehrl, L. Ruttiger, S.C. Beck, F. Tonagel, B.J. Pichler, M. Knipper, T. Peters, J. Laufs, and B. Wissinger. 2007. A splice site mutation in the murine Opa1 gene features pathology of autosomal dominant optic atrophy. Brain. 130:1029-1042. doi:10.1093/brain/awm005.

Alexander, C., M. Votruba, U.E. Pesch, D.L. Thiselton, S. Mayer, A. Moore, M. Rodriguez, U. Kellner, B. Leo-Kottler, G. Auburger, S.S. Bhattacharya, and B. Wissinger. 2000a. OPA1, encoding a dynamin-related GTPase, is mutated in autosomal dominant optic atrophy linked to chromosome 3q28. Nat. Genet. 26:211-215. doi:10.1038/79944.

Anand, R., T. Wai, M.J. Baker, N. Kladt, A.C. Schauss, E. Rugarli, and T. Langer. 2014a. The iAAA protease YME1L and OMA1 cleave OPA1 to balance mitochondrial fusion and fission. J Cell Biol. 204:919-929. doi:10.1083/jcb.201308006.

Ban, T., T. Ishihara, H. Kohno, S. Saita, A. Ichimura, K. Maenaka, T. Oka, K. Mihara, and N. Ishihara. 2017a. Molecular basis of selective mitochondrial fusion by heterotypic action between OPA1 and cardiolipin. Nat Cell Biol. 19:856-863. doi:10.1038/ncb3560.

Ban, T., H. Kohno, T. Ishihara, and N. Ishihara. 2018a. Relationship between OPA1 and cardiolipin in mitochondrial inner-membrane fusion. Biochim Biophys Acta Bioenerg. 1859:951-957. doi:10.1016/j.bbabio.2018.05.016.

Ban-Ishihara, R., T. Ishihara, N. Sasaki, K. Mihara, and N. Ishihara. 2013. Dynamics of nucleoid structure regulated by mitochondrial fission contributes to cristae reformation and release of cytochrome c. Proc. Natl. Acad. Sci. U.S.A. 110:11863-11868. doi:10.1073/pnas.1301951110.

Barrera, M., S. Koob, D. Dikov, F. Vogel, and A.S. Reichert. 2016. OPA1 functionally interacts with MIC60 but is dispensable for crista junction formation. FEBS Lett. 590:3309-3322. doi:10.1002/1873-3468.12384.

Bayrhuber, M., T. Meins, M. Habeck, S. Becker, K. Giller, S. Villinger, C. Vonrhein, C. Griesinger, M. Zweckstetter, and K. Zeth. 2008. Structure of the human voltagedependent anion channel. Proc. Natl. Acad. Sci. U.S.A. 105:15370-15375. doi:10.1073/pnas.0808115105.

Bleazard, W., J.M. McCaffery, E.J. King, S. Bale, A. Mozdy, Q. Tieu, J. Nunnari, and J.M. Shaw. 1999. The dynamin-related GTPase Dnm1 regulates mitochondrial fission in yeast. Nat. Cell Biol. 1:298-304. doi:10.1038/13014.

de Brito, O.M., and L. Scorrano. 2008. Mitofusin 2 tethers endoplasmic reticulum to mitochondria. Nature. 456:605-610. doi:10.1038/nature07534. 
Carelli, V., and D.C. Chan. 2014. Mitochondrial DNA: impacting central and peripheral nervous systems. Neuron. 84:1126-1142. doi:10.1016/j.neuron.2014.11.022.

Chan, D.C. 2020. Mitochondrial Dynamics and Its Involvement in Disease. Annu. Rev. Pathol. Mech. Dis. 15:235-259. doi:10.1146/annurev-pathmechdis-012419-032711.

Chen, H., A. Chomyn, and D.C. Chan. 2005b. Disruption of Fusion Results in Mitochondrial Heterogeneity and Dysfunction. J. Biol. Chem. 280:26185-26192. doi:10.1074/jbc.M503062200.

Chen, H., S.A. Detmer, A.J. Ewald, E.E. Griffin, S.E. Fraser, and D.C. Chan. 2003a. Mitofusins Mfn1 and Mfn2 coordinately regulate mitochondrial fusion and are essential for embryonic development. J Cell Biol. 160:189-200. doi:10.1083/jcb.200211046.

Chen, H., J.M. McCaffery, and D.C. Chan. 2007. Mitochondrial fusion protects against neurodegeneration in the cerebellum. Cell. 130:548-62. doi:10.1016/j.cell.2007.06.026.

Chen, H., M. Vermulst, Y.E. Wang, A. Chomyn, T.A. Prolla, J.M. McCaffery, and D.C. Chan. 2010. Mitochondrial fusion is required for mtDNA stability in skeletal muscle and tolerance of mtDNA mutations. Cell. 141:280-289. doi:10.1016/j.cell.2010.02.026.

Cipolat, S., O. Martins de Brito, B. Dal Zilio, and L. Scorrano. 2004. OPA1 requires mitofusin 1 to promote mitochondrial fusion. Proc. Natl. Acad. Sci. U.S.A. 101:15927-15932. doi:10.1073/pnas.0407043101.

Cogliati, S., C. Frezza, M.E. Soriano, T. Varanita, R. Quintana-Cabrera, M. Corrado, S. Cipolat, V. Costa, A. Casarin, L.C. Gomes, E. Perales-Clemente, L. Salviati, P. Fernandez-Silva, J.A. Enriquez, and L. Scorrano. 2013. Mitochondrial cristae shape determines respiratory chain supercomplexes assembly and respiratory efficiency. Cell. 155:160-171. doi:10.1016/j.cell.2013.08.032.

Cole, L.W. 2016. The Evolution of Per-cell Organelle Number. Front Cell Dev Biol. 4. doi:10.3389/fcell.2016.00085.

Crompton, M., and A. Costi. 1990. A heart mitochondrial Ca2+-dependent pore of possible relevance to re-perfusion-induced injury. Evidence that ADP facilitates pore interconversion between the closed and open states. Biochem J. 266:33-39. doi:10.1042/bj2660033.

Del Dotto, V., P. Mishra, S. Vidoni, M. Fogazza, A. Maresca, L. Caporali, J.M. McCaffery, M. Cappelletti, E. Baruffini, G. Lenaers, D. Chan, M. Rugolo, V. Carelli, and C. Zanna. 2017a. OPA1 Isoforms in the Hierarchical Organization of Mitochondrial Functions. Cell Rep. 19:2557-2571. doi:10.1016/j.celrep.2017.05.073.

Delettre, C., J.M. Griffoin, J. Kaplan, H. Dollfus, B. Lorenz, L. Faivre, G. Lenaers, P. Belenguer, and C.P. Hamel. 2001. Mutation spectrum and splicing variants in the OPA1 gene. Hum. Genet. 109:584-591. doi:10.1007/s00439-001-0633-y. 
Delettre, C., G. Lenaers, J.-M. Griffoin, N. Gigarel, C. Lorenzo, P. Belenguer, L. Pelloquin, J. Grosgeorge, C. Turc-Carel, E. Perret, C. Astarie-Dequeker, L. Lasquellec, B. Arnaud, B. Ducommun, J. Kaplan, and C.P. Hamel. 2000a. Nuclear gene OPA1, encoding a mitochondrial dynamin-related protein, is mutated in dominant optic atrophy. Nature Genetics. 26:207-210. doi:10.1038/79936.

DeVay, R.M., L. Dominguez-Ramirez, L.L. Lackner, S. Hoppins, H. Stahlberg, and J. Nunnari. 2009. Coassembly of Mgm1 isoforms requires cardiolipin and mediates mitochondrial inner membrane fusion. J. Cell Biol. 186:793-803. doi:10.1083/jcb.200906098.

Ehses, S., I. Raschke, G. Mancuso, A. Bernacchia, S. Geimer, D. Tondera, J.-C. Martinou, B. Westermann, E.I. Rugarli, and T. Langer. 2009. Regulation of OPA1 processing and mitochondrial fusion by m-AAA protease isoenzymes and OMA1. J. Cell Biol. 187:1023-1036. doi:10.1083/jcb.200906084.

Elachouri, G., S. Vidoni, C. Zanna, A. Pattyn, H. Boukhaddaoui, K. Gaget, P. Yu-Wai-Man, G. Gasparre, E. Sarzi, C. Delettre, A. Olichon, D. Loiseau, P. Reynier, P.F. Chinnery, A. Rotig, V. Carelli, C.P. Hamel, M. Rugolo, and G. Lenaers. 2011. OPA1 links human mitochondrial genome maintenance to mtDNA replication and distribution. Genome Res. 21:12-20. doi:10.1101/gr.108696.110.

Faelber, K., L. Dietrich, J.K. Noel, F. Wollweber, A.-K. Pfitzner, A. Mühleip, R. Sánchez, M. Kudryashev, N. Chiaruttini, H. Lilie, J. Schlegel, E. Rosenbaum, M. Hessenberger, C. Matthaeus, S. Kunz, A. von der Malsburg, F. Noé, A. Roux, M. van der Laan, W. Kühlbrandt, and O. Daumke. 2019. Structure and assembly of the mitochondrial membrane remodelling GTPase Mgm1. Nature. 571:429-433. doi:10.1038/s41586-0191372-3.

Focusing on mitochondrial form and function. 2018. Nat Cell Biol. 20:735-735. doi:10.1038/s41556-018-0139-7.

Fonseca, T.B., Á. Sánchez-Guerrero, I. Milosevic, and N. Raimundo. 2019. Mitochondrial fission requires DRP1 but not dynamins. Nature. 570:E34-E42. doi:10.1038/s41586-0191296-y.

Frezza, C., S. Cipolat, O.M. de Brito, M. Micaroni, G.V. Beznoussenko, T. Rudka, D. Bartoli, R.S. Polishuck, N.N. Danial, B.D. Strooper, and L. Scorrano. 2006. OPA1 controls apoptotic cristae remodeling independently from mitochondrial fusion. Cell. 126:177189. doi:10.1016/j.cell.2006.06.025.

Friedman, J.R., L.L. Lackner, M. West, J.R. DiBenedetto, J. Nunnari, and G.K. Voeltz. 2011. ER tubules mark sites of mitochondrial division. Science. 334:358-362.

doi:10.1126/science.1207385.

Fröhlich, C., S. Grabiger, D. Schwefel, K. Faelber, E. Rosenbaum, J. Mears, O. Rocks, and O. Daumke. 2013. Structural insights into oligomerization and mitochondrial remodelling of dynamin 1-like protein. The EMBO Journal. 32:1280-1292. doi:10.1038/emboj.2013.74. 
Gandre-Babbe, S., and A.M. van der Bliek. 2008. The novel tail-anchored membrane protein Mff controls mitochondrial and peroxisomal fission in mammalian cells. $M B o C$. 19:24022412. doi:10.1091/mbc.e07-12-1287.

Ge, Y., X. Shi, S. Boopathy, J. McDonald, A.W. Smith, and L.H. Chao. 2020. Two forms of Opal cooperate to complete fusion of the mitochondrial inner-membrane. eLife. 9:e50973. doi:10.7554/eLife.50973.

Gilkerson, R.W., E.A. Schon, E. Hernandez, and M.M. Davidson. 2008. Mitochondrial nucleoids maintain genetic autonomy but allow for functional complementation. J. Cell Biol. 181:1117-1128. doi:10.1083/jcb.200712101.

Glater, E.E., L.J. Megeath, R.S. Stowers, and T.L. Schwarz. 2006. Axonal transport of mitochondria requires milton to recruit kinesin heavy chain and is light chain independent. J. Cell Biol. 173:545-557. doi:10.1083/jcb.200601067.

Gomes, L.C., G. Di Benedetto, and L. Scorrano. 2011. During autophagy mitochondria elongate, are spared from degradation and sustain cell viability. Nat. Cell Biol. 13:589-598. doi:10.1038/ncb2220.

Griparic, L., T. Kanazawa, and A.M. van der Bliek. 2007a. Regulation of the mitochondrial dynamin-like protein Opal by proteolytic cleavage. J. of Cell Biol. 178:757-764. doi:10.1083/jcb.200704112.

Hales, K.G., and M.T. Fuller. 1997. Developmentally regulated mitochondrial fusion mediated by a conserved, novel, predicted GTPase. Cell. 90:121-129. doi:10.1016/S00928674(00)80319-0.

Head, B., L. Griparic, M. Amiri, S. Gandre-Babbe, and A.M. van der Bliek. 2009. Inducible proteolytic inactivation of OPA1 mediated by the OMA1 protease in mammalian cells. The J. of Cell Biol. 187:959-966. doi:10.1083/jcb.200906083.

Hoppel, C.L., B. Tandler, H. Fujioka, and A. Riva. 2009. Dynamic organization of mitochondria in human heart and in myocardial disease. Int. J. Biochem. Cell Biol. 41:1949-1956. doi:10.1016/j.biocel.2009.05.004.

Hwa, J.J., M.A. Hiller, M.T. Fuller, and A. Santel. 2002. Differential expression of the Drosophila mitofusin genes fuzzy onions (fzo) and dmfn. Mech. Dev. 116:213-216. doi:10.1016/s0925-4773(02)00141-7.

Ishihara, N., Y. Eura, and K. Mihara. 2004. Mitofusin 1 and 2 play distinct roles in mitochondrial fusion reactions via GTPase activity. J. of Cell Sci. 117:6535-6546. doi:10.1242/jcs.01565.

Ishihara, N., M. Nomura, A. Jofuku, H. Kato, S.O. Suzuki, K. Masuda, H. Otera, Y. Nakanishi, I. Nonaka, Y. Goto, N. Taguchi, H. Morinaga, M. Maeda, R. Takayanagi, S. Yokota, and K. Mihara. 2009. Mitochondrial fission factor Drp1 is essential for embryonic 
development and synapse formation in mice. Nat. Cell Biol.. 11:958-966. doi:10.1038/ncb1907.

Ishihara, T., R. Ban-Ishihara, M. Maeda, Y. Matsunaga, A. Ichimura, S. Kyogoku, H. Aoki, S. Katada, K. Nakada, M. Nomura, N. Mizushima, K. Mihara, and N. Ishihara. 2015. Dynamics of mitochondrial DNA nucleoids regulated by mitochondrial fission is essential for maintenance of homogeneously active mitochondria during neonatal heart development. Mol. Cell. Biol. 35:211-223. doi:10.1128/MCB.01054-14.

Karbowski, M., M.M. Cleland, and B.A. Roelofs. 2014. Photoactivatable green fluorescent protein-based visualization and quantification of mitochondrial fusion and mitochondrial network complexity in living cells. Meth. Enzymol. 547:57-73. doi:10.1016/B978-0-12801415-8.00004-7.

Knott, A.B., and E. Bossy-Wetzel. 2008. Impairing the mitochondrial fission and fusion Balance: A new mechanism of neurodegeneration. Ann N Y Acad Sci. 1147:283-292. doi:10.1196/annals.1427.030.

Koch, A., M. Thiemann, M. Grabenbauer, Y. Yoon, M.A. McNiven, and M. Schrader. 2003. Dynamin-like protein 1 is involved in peroxisomal fission. J. Biol. Chem. 278:85978605. doi:10.1074/jbc.M211761200.

Kondadi, A.K., R. Anand, S. Hänsch, J. Urbach, T. Zobel, D.M. Wolf, M. Segawa, M. Liesa, O.S. Shirihai, S. Weidtkamp-Peters, and A.S. Reichert. 2020. Cristae undergo continuous cycles of membrane remodelling in a MICOS-dependent manner. EMBO reports. 21:e49776. doi:10.15252/embr.201949776.

Kühlbrandt, W. 2015. Structure and function of mitochondrial membrane protein complexes. BMC Biol. 13. doi:10.1186/s12915-015-0201-x.

Labrousse, A.M., M.D. Zappaterra, D.A. Rube, and A.M. van der Bliek. 1999. C. elegans Dynamin-Related Protein DRP-1 Controls Severing of the Mitochondrial Outer Membrane. Molecular Cell. 4:815-826. doi:10.1016/S1097-2765(00)80391-3.

Lee, H., S.B. Smith, S.-S. Sheu, and Y. Yoon. 2020a. The short variant of optic atrophy 1 (OPA1) improves cell survival under oxidative stress. J. Biol. Chem. jbc.RA119.010983. doi:10.1074/jbc.RA119.010983.

Lee, H., S.B. Smith, and Y. Yoon. 2017a. The short variant of the mitochondrial dynamin OPA1 maintains mitochondrial energetics and cristae structure. J. Biol. Chem. 292:7115-7130. doi:10.1074/jbc.M116.762567.

Lee, J.E., L.M. Westrate, H. Wu, C. Page, and G.K. Voeltz. 2016. Multiple dynamin family members collaborate to drive mitochondrial division. Nature. 540:139-143. doi:10.1038/nature20555. 
Li, Z., K.-I. Okamoto, Y. Hayashi, and M. Sheng. 2004. The importance of dendritic mitochondria in the morphogenesis and plasticity of spines and synapses. Cell. 119:873887. doi:10.1016/j.cell.2004.11.003.

Liu, X., D. Weaver, O. Shirihai, and G. Hajnóczky. 2009. Mitochondrial "kiss-and-run": interplay between mitochondrial motility and fusion-fission dynamics. EMBO J. 28:3074-3089. doi:10.1038/emboj.2009.255.

Losón, O.C., Z. Song, H. Chen, and D.C. Chan. 2013. Fis1, Mff, MiD49, and MiD51 mediate Drp1 recruitment in mitochondrial fission. $M B o C .24: 659-667$. doi:10.1091/mbc.e12-100721.

Mears, J.A., L.L. Lackner, S. Fang, E. Ingerman, J. Nunnari, and J.E. Hinshaw. 2011. Conformational changes in Dnm1 support a contractile mechanism for mitochondrial fission. Nature Structural \& Molecular Biology. 18:20-26. doi:10.1038/nsmb.1949.

Menzies, R.A., and P.H. Gold. 1971. The turnover of mitochondria in a variety of tissues of young adult and aged rats. J. Biol. Chem. 246:2425-2429.

Mishra, P., V. Carelli, G. Manfredi, and D.C. Chan. 2014b. Proteolytic cleavage of Opa1 stimulates mitochondrial inner membrane fusion and couples fusion to oxidative phosphorylation. Cell Metab. 19:630-641. doi:10.1016/j.cmet.2014.03.011.

Misko, A., S. Jiang, I. Wegorzewska, J. Milbrandt, and R.H. Baloh. 2010. Mitofusin 2 is necessary for transport of axonal mitochondria and interacts with the Miro/Milton complex. J. Neurosci. 30:4232-4240. doi:10.1523/JNEUROSCI.6248-09.2010.

Olichon, A., L. Baricault, N. Gas, E. Guillou, A. Valette, P. Belenguer, and G. Lenaers. 2003. Loss of OPA1 perturbates the mitochondrial inner membrane structure and integrity, leading to cytochrome c release and apoptosis. J. Biol. Chem. 278:7743-7746. doi:10.1074/jbc.C200677200.

Olichon, A., G. Elachouri, L. Baricault, C. Delettre, P. Belenguer, and G. Lenaers. 2007. OPA1 alternate splicing uncouples an evolutionary conserved function in mitochondrial fusion from a vertebrate restricted function in apoptosis. Cell Death Differ. 14:682-692. doi:10.1038/sj.cdd.4402048.

Otera, H., C. Wang, M.M. Cleland, K. Setoguchi, S. Yokota, R.J. Youle, and K. Mihara. 2010. Mff is an essential factor for mitochondrial recruitment of Drp1 during mitochondrial fission in mammalian cells. J. Cell Biol. 191:1141-1158. doi:10.1083/jcb.201007152.

Palmer, C.S., L.D. Osellame, D. Laine, O.S. Koutsopoulos, A.E. Frazier, and M.T. Ryan. 2011. MiD49 and MiD51, new components of the mitochondrial fission machinery. EMBO reports. 12:565-573. doi:10.1038/embor.2011.54.

Patten, D.A., J. Wong, M. Khacho, V. Soubannier, R.J. Mailloux, K. Pilon-Larose, J.G. MacLaurin, D.S. Park, H.M. McBride, L. Trinkle-Mulcahy, M.-E. Harper, M. Germain, and R.S. Slack. 2014. OPA1-dependent cristae modulation is essential for cellular 
adaptation to metabolic demand. EMBO J. 33:2676-2691.

doi:10.15252/embj.201488349.

Pilling, A.D., D. Horiuchi, C.M. Lively, and W.M. Saxton. 2006. Kinesin-1 and dynein are the primary motors for fast transport of mitochondria in Drosophila motor axons. $M B O C$. 17:2057-2068. doi:10.1091/mbc.e05-06-0526.

Rambold, A.S., B. Kostelecky, N. Elia, and J. Lippincott-Schwartz. 2011. Tubular network formation protects mitochondria from autophagosomal degradation during nutrient starvation. Proc. Natl. Acad. Sci. U.S.A. 108:10190-10195. doi:10.1073/pnas.1107402108.

Ran, F.A., P.D. Hsu, J. Wright, V. Agarwala, D.A. Scott, and F. Zhang. 2013. Genome engineering using the CRISPR-Cas9 system. Nature Protocols. 8:2281-2308. doi:10.1038/nprot.2013.143.

Riva, A., B. Tandler, F. Loffredo, E. Vazquez, and C. Hoppel. 2005. Structural differences in two biochemically defined populations of cardiac mitochondria. Am. J. Physiol. Heart Circ. Physiol. 289:H868-872. doi:10.1152/ajpheart.00866.2004.

Rojo, M., F. Legros, D. Chateau, and A. Lombès. 2002. Membrane topology and mitochondrial targeting of mitofusins, ubiquitous mammalian homologs of the transmembrane GTPase Fzo. Journal of Cell Science. 115:1663-1674.

Russo, G.J., K. Louie, A. Wellington, G.T. Macleod, F. Hu, S. Panchumarthi, and K.E. Zinsmaier. 2009. Drosophila Miro is required for both anterograde and retrograde axonal mitochondrial transport. J. Neurosci. 29:5443-5455. doi:10.1523/JNEUROSCI.541708.2009 .

Santel, A., and M.T. Fuller. 2001. Control of mitochondrial morphology by a human mitofusin. Journal of Cell Science. 114:867-874.

Shi, H., A.J. Rampello, and S.E. Glynn. 2016a. Engineered AAA+ proteases reveal principles of proteolysis at the mitochondrial inner membrane. Nat Commun. 7:13301. doi:10.1038/ncomms13301.

Smirnova, E., L. Griparic, D.-L. Shurland, and A.M. van der Bliek. 2001. Dynamin-related Protein Drp1 Is Required for Mitochondrial Division in Mammalian Cells. MBoC. 12:2245-2256. doi:10.1091/mbc.12.8.2245.

Song, Z., H. Chen, M. Fiket, C. Alexander, and D.C. Chan. 2007a. OPA1 processing controls mitochondrial fusion and is regulated by mRNA splicing, membrane potential, and Yme1L. J. Cell Biol. 178:749-755. doi:10.1083/jcb.200704110.

Song, Z., M. Ghochani, J.M. McCaffery, T.G. Frey, and D.C. Chan. 2009. Mitofusins and OPA1 Mediate Sequential Steps in Mitochondrial Membrane Fusion. Mol Biol Cell. 20:35253532. doi:10.1091/mbc.E09-03-0252. 
Stowers, R.S., L.J. Megeath, J. Górska-Andrzejak, I.A. Meinertzhagen, and T.L. Schwarz. 2002. Axonal transport of mitochondria to synapses depends on milton, a novel Drosophila protein. Neuron. 36:1063-1077. doi:10.1016/s0896-6273(02)01094-2.

Sun, M.G., J. Williams, C. Munoz-Pinedo, G.A. Perkins, J.M. Brown, M.H. Ellisman, D.R. Green, and T.G. Frey. 2007. Correlated three-dimensional light and electron microscopy reveals transformation of mitochondria during apoptosis. Nature Cell Biology. 9:10571065. doi:10.1038/ncb1630.

Takeyama, N., N. Matsuo, and T. Tanaka. 1993. Oxidative damage to mitochondria is mediated by the Ca2+-dependent inner-membrane permeability transition. Biochem J. 294:719725. doi:10.1042/bj2940719.

Tanaka, A., M.M. Cleland, S. Xu, D.P. Narendra, D.-F. Suen, M. Karbowski, and R.J. Youle. 2010. Proteasome and p97 mediate mitophagy and degradation of mitofusins induced by Parkin. J Cell Biol. 191:1367-1380. doi:10.1083/jcb.201007013.

Tilokani, L., S. Nagashima, V. Paupe, and J. Prudent. 2018. Mitochondrial dynamics: overview of molecular mechanisms. Essays in Biochemistry. 62:341-360. doi:10.1042/EBC20170104.

Tondera, D., S. Grandemange, A. Jourdain, M. Karbowski, Y. Mattenberger, S. Herzig, S. Da Cruz, P. Clerc, I. Raschke, C. Merkwirth, S. Ehses, F. Krause, D.C. Chan, C. Alexander, C. Bauer, R. Youle, T. Langer, and J.-C. Martinou. 2009b. SLP-2 is required for stressinduced mitochondrial hyperfusion. EMBO J. 28:1589-1600. doi:10.1038/emboj.2009.89.

Twig, G., A. Elorza, A.J.A. Molina, H. Mohamed, J.D. Wikstrom, G. Walzer, L. Stiles, S.E. Haigh, S. Katz, G. Las, J. Alroy, M. Wu, B.F. Py, J. Yuan, J.T. Deeney, B.E. Corkey, and O.S. Shirihai. 2008. Fission and selective fusion govern mitochondrial segregation and elimination by autophagy. EMBO J. 27:433-446. doi:10.1038/sj.emboj.7601963.

Verstreken, P., C.V. Ly, K.J.T. Venken, T.-W. Koh, Y. Zhou, and H.J. Bellen. 2005. Synaptic Mitochondria Are Critical for Mobilization of Reserve Pool Vesicles at Drosophila Neuromuscular Junctions. Neuron. 47:365-378. doi:10.1016/j.neuron.2005.06.018.

Wai, T., J. García-Prieto, M.J. Baker, C. Merkwirth, P. Benit, P. Rustin, F.J. Rupérez, C. Barbas, B. Ibañez, and T. Langer. 2015. Imbalanced OPA1 processing and mitochondrial fragmentation cause heart failure in mice. Science. 350:aad0116. doi:10.1126/science.aad0116.

Yamaguchi, R., L. Lartigue, G. Perkins, R.T. Scott, A. Dixit, Y. Kushnareva, T. Kuwana, M. Ellisman, and D.D. Newmeyer. 2008. Opa1-mediated cristae opening is Bax/Bak- and BH3-dependent, required for apoptosis, and independent of Bak oligomerization. Mol Cell. 31:557-569. doi:10.1016/j.molcel.2008.07.010.

Zhao, J., T. Liu, S. Jin, X. Wang, M. Qu, P. Uhlén, N. Tomilin, O. Shupliakov, U. Lendahl, and M. Nistér. 2011. Human MIEF1 recruits Drp1 to mitochondrial outer membranes and 
promotes mitochondrial fusion rather than fission. The EMBO J.. 30:2762-2778. doi:10.1038/emboj.2011.198. 


\section{Chapter 3}

\section{Discussion}

This work has demonstrated the following points:

1. Opal is proteolytically cleaved in a novel site by a known protease.

2. This cleavage is complete under basal conditions whenever exon $4 \mathrm{~b}$ is present in an isoform.

3. The $\mathrm{S} 3$ cleaved isoform alone cannot promote fusion, but together with a long isoform, it can enhance mitochondrial tubulation.

Several key issues still remain to be solved:

Why would OPA1 need the additional cleavage site? What is the physiological function of the S3 site, especially given the fact that the existence of the site can lead to complete OPA1 processing? What is the structural significance of exon $4 \mathrm{~b}$ ? In comparison with exon $5 \mathrm{~b}$, which both serve as YME1L recognition sites, a better understanding of the structural difference between those two sites will not only enable us to understand OPA1 structure better, but also to elucidate more on YME1L's proteolytic mechanism.

It has been previously well established that OPAl protein has two cleavage sites in the main stalk in intermembrane space, generating three different short isoforms. With my study, we confirmed with solid evidence that a third cleavage site exists in exon $4 \mathrm{~b}$ which is present in half of the isoforms. Isoforms with exon $4 \mathrm{~b}$ expression are completely processed into short forms by the m-AAA protease YME11, which is well known to cleave at S2. The complete cleavage seems to be quite a strong effect on the protein given that half of the isoforms are completely processed 
at the site. Why Opa1 has evolved such a complicated cleavage system is not quite well understood. The alternative splicing mechanism only exists in higher vertebrates, and may be a product of evolution. Therefore, it is not difficult to imagine that the existence of this complicated proteolytic processing can be a protective mechanism for Opa1 to maintain a system where at least a proportion of OPA1 can be post-translationally processed at at least one site under most circumstances, even when the function of one protease gets disrupted or some isoforms of Opa1 are not expressed well.

Mgm1, the yeast homolog of Opa1, consists of a GTPase (G) domain, a bundle signaling element domain, a stalk, and a paddle domain that contains a membrane-binding site (Faelber et al., 2019). Biochemical and cell-based experiments demonstrate that the Mgm1 stalk mediates the assembly of bent tetramers into helical filaments. Electron cryo-tomography studies of Mgm1-decorated lipid tubes and fluorescence microscopy experiments on reconstituted membrane tubes indicate how the tetramers assemble on positively or negatively curved membranes (Faelber et al., 2019). Based on these studies, it seems that the membrane unassociated s-OPA1 have fusion capability by forming tubes and coordinate with the 1-OPA1 to pull the inner membrane open.

\section{Selectivity of YME1L proteolytic activity on OPA1}

It is interesting to continue understanding how YME1L can distinguish between S3 and $\mathrm{S} 2$, especially when both sites are present in one isoform. It remains to be determined why inclusion of exon $4 \mathrm{~b}$ causes such extensive processing by YME1L. A short hydrophobic sequence (FAWFP) has been found to act as a degron for YME1L (Shi et al., 2016b). However, 
no similar sequences are apparent in exon $4 \mathrm{~b}$ or the rest of OPA1. The exact sequence of S2 site is still unknown. Our data suggest that the $\mathrm{S} 2$ site exists in the LILL region in exon5b, which is also highly enriched in leucines (not published). Based on the similarity of the sequence, it is likely that YME1L does not distinguish the site based on sequence. One possibility is the spatial location, where YME1L cleaves at the first site it comes across. This is less likely since we know that when S3 does not exist, YME1L cleaves at S2 but incompletely, leading to only part of the long isoform generating a S2 short form. Whenever exon4b exists in the isoform, Yme1L can instead completely cleave the long form all into short forms. Therefore, another more likely explanation would be that the structure of exon $4 \mathrm{~b} / \mathrm{S} 3$ provides a better access of Yme1L to the Opa1 isoform and somehow facilitates the processing capability. Although recent study reveals a partial OPA1 structure starting from the G domain region, the authors suggest that the alternative splicing region is predicted to be unstructured and difficult to resolve. Future structural data are needed to better understand this mechanism, which will not only help better understand the OPA1 processing but also the Yme1L cleavage mechanism, which is a uniquely important inner membrane protease known to have multiple substrates on the inner membrane, and may be involved in essential cellular functions, proved by the embryonic lethality when knocked out in mouse.

Another intriguing question is whether YME1L distinguish between the two sites for different functions, in particular responding to various cellular stress. A previous study done in our lab suggests that the YME1L cleavage at S2 site is responsive to OXPHOS stress stimulation. When cells are forced to do pure OXPHOS to generate ATP (no glycolysis), cleavage at S2 gets enhanced and vice versa. When artificially cleaving at S2, mitochondrial 
inner membrane fusion is induced in vitro (Mishra et al., 2014a). There has been no obvious

evidence that the S3 serves the same function, as we did observe OXPHOS-induced elongation in exon $4 \mathrm{~b}$ knockdown cells. It is possible that the S3 site can be induced by another stress cue and YME1L cleavage can further coordinate according to different environmental stimuli. A recent study shows that cells expressing S3 cleaved short isoform only show better survival under oxidant insult in OXPHOS media compared to cells with only a non-cleavable long isoform 1, which mainly involves necrosis (Lee et al., 2020a). The authors propose a model where short Opa1 can cast a protective effect on cell survival under certain stress conditions, explaining the pronounced production of short Opa1 when cells are challenged with some external stimuli.

\section{Functions of S3 cleaved s-OPA1, fusion vs cristae/OXPHOS maintenance?}

Our findings suggest that this short Opal isoform contributes to fusion activity when expressed together with a long-form generating isoform. When expressing isoform 5 together with isoform 1 in Opal-null MEFs, the mitochondria are more tubulated compared to those in isoform 1 expressing MEFs alone. It seems like this short form exhibits an additive function to overall opa1 fusion activity. The persistence of the cleavage events on OPA1 also suggest that the production of short OPA1 seem to be fundamental for mitochondria and therefore evolved such a well maintained mechanism to keep this process. A previous idea where long form OPA1 being the functional unit and s-OPA1 is detrimental to cells seems to be inaccurate, as it is not beneficial for cells to spend energy on such a useless event. 


\section{The role distinguishing different s-OPA1 forms, if there is any}

It is now clear that the short forms of OPA1 can be roughly categorized into two typesOMA1-generated short forms and YME1L-generated short forms. In the YME1L generated short forms, it is more complicated in terms of actual cleavage site-exon $4 b$, exon $5 b$, and when both disrupted, potentially in exon 4. The reason why YME1L can recognize and cleave at so many different sites remains unknown. With different sizes and N-term residues, it is not known whether these different s-OPA1 forms have various functions from each other. It seems unlikely that with so few amino acid residue differences, there may be any major physiological function change, however, more detailed experiments are still needed to verify this point. Previous results from two independent studies have suggested that s-OPA1 seem to share similar functions in mtDNA maintenance, cristae structure, etc.

According to a contradictory result from Ban et al, additional analysis is needed to understand the molecular properties of s-OPA1 (e.g., assembly state and orientation), both in solution and on the membrane.

\section{Which is more important, absolute or relative amount of s-OPA1?}

It is still not clear whether the absolute amount of OPA1 matters more for fusion and other activities, or the relative amount of short compared to long OPA1 is more important.

So far, the model for OPA1 mediated inner membrane fusion seems to be that the GTP hydrolysis process functions as a powerful stroke and when hydrolyzed, strongly pull the inner 
membrane together to facilitate fusion. The GTP hydrolysis activity gets dramatically enhanced by interacting with cardiolipin, which is in high concentration on the inner membrane. The reason that various in vitro studies suggest that 1-OPA1 with cardiolipin alone can promote fusion whereas in vivo studies show that the existence of both are needed to complete fusion may be explained by the absolute amount needed for mediating fusion. In one study, the authors found that with a fixed amount of OPA1, the higher the 1-OPA1 content, the more fusion, and vice versa. When only the 1-OPA1 level is kept constant, the more s-OPA1 was added to the reaction (therefore the total amount of OPA1 increases), the more fusion events were observed.

A structural study revealed that both 1- and s-OPA1 can form tetramers and further tubulate, as the tubulating domain exists in the middle stalk that is in the middle to C-term end of OPA1. When membrane anchored 1-Opa1 is enriched, the more free-flowing s-OPA1, the better fusion activity it may possess as the free s-OPA1 can freely bind to any 1-OPA1 that need to tubulate to fuse; however, when the binding gets saturated, the upper limit of fusion gets reached and by adding 1-Opa1 at the same time, the fusion limit gets uplifted. The difference shown between in vivo vs in vitro systems likely resides in the absolute amount of OPA1. In live cells, the amount of OPA1 is tightly regulated. At any given time without external stimuli, the amount of Opal mRNAs and proteins are limited and may not show obvious variations. Therefore, a tight regulation of s-OPA1 production is needed to fulfill the need to reach an optimal fusion level. If all OPA1 remains as long forms and anchored on the inner membrane, the lack of free OPA1 substrate prevents tetramer and tubule formation of the proteins; if excessive s-OPA1 is produced, without membrane anchor partner (1-OPA1), the formed free tetramer cannot properly bind to the membrane to mediate fusion either. 
It is important to notice that previous studies and our data all prove that over-expressing Opal can lead to mitochondrial fragmentation, even with the long form generating isoforms. It is possible that a mechanism exists that when OPA1 concentration is too high, fusion activity get inhibited. It can be a mechanism where the inner membrane protects from fusing with each other, especially in cristae junction regions where Opal can exist on opposing sides of the same inner membrane.

An interesting new study done by Ge et al. established an in vitro assay to visualize the inner membrane fusion more closely on a single event basis. In their study, they further broke down one single inner membrane fusion process into four steps: membrane tethering, docking, hemifusion, and pore opening. They found that while both 1- and s-OPA1 can complete the first tethering step, where membranes are brought into close proximity, only 1-OPA1 can further mediate the docking step, where tethered membranes are brought into extremely close distance (FRET distance, <40-60 A) and ready to progress into fusion (Ge et al., 2020). Furthermore, 1OPA1 on a heterotypic membrane (in this case, only one membrane is anchored with 1-OPA1 protein and the other is free of OPA1) can progress further into lipid bilayer hemifusion, however, the efficiency of hemifusion is at least 3-folds lower compared to that of 1-OPA1 on a homotypic membrane (1-OPA1 expressed on both lipid bilayers). The final step of fusion is pore opening and content release, where both leaflets merge. The authors found that about half of the particles undergoing hemifusion can proceed to pore opening (Ge et al., 2020). S-OPA1 labeled particles or heterotypic 1-OPA1 particles do not show pore opening or content mixing. Equimolar addition of s-OPA1 into the 1-OPA1 homotypic particle system increases the pore opening efficiency by at least eight-fold and decreases the dwell time by four-fold. Excessive s-OPA1 
have a dominant negative effect to this process and have been shown to facilitate vesicle detachment of the 1-OPA1 particles.

\section{The interplay between OPA1 and mitochondrial permeability transition}

Mitochondrial permeability transition is an alteration in mitochondrial inner membrane permeability, which is usually highly nonpermeable. It is thought to be the strategic regulator of

cell death when first discovered (Crompton and Costi, 1990), and can be induced by $\mathrm{Ca}^{2+}$ as well as oxidative stress (Takeyama et al., 1993).

A recent study on OPA1 short isoform showed that when cells are supplemented with only short OPA1, they can decrease the sensitivity of MPT, leading to a 'protective' effect on cells under oxidative insults (Lee et al., 2020a). The opposite effect was seen with cells expressing solely exogenous 1-OPA1, which sensitize the cells under oxidative stress and which results in early membrane permeability collapse. This new finding opens up the path to think possible additional roles of OPA1 besides mediating fusion and cristae remodeling, and extends the level of the OPA1 function to a broader scheme within the mitochondrial microenvironment. However, the researchers were not able to elucidate the pathway that OPA1 may be playing a role in, and the functional partner (if any) of OPA1. The here introduced in vivo model of 1- and s-OPA1 isoforms and their combination would be able to test this MPT sensitization function of endogenous OPA1 under various stress conditions, and further study the link between OPA1 and other inner membrane components. 


\section{Reference}

Crompton, M., and A. Costi. 1990. A heart mitochondrial Ca2+-dependent pore of possible relevance to re-perfusion-induced injury. Evidence that ADP facilitates pore interconversion between the closed and open states. Biochem. J. 266:33-39. doi:10.1042/bj2660033.

Faelber, K., L. Dietrich, J.K. Noel, F. Wollweber, A.-K. Pfitzner, A. Mühleip, R. Sánchez, M. Kudryashev, N. Chiaruttini, H. Lilie, J. Schlegel, E. Rosenbaum, M. Hessenberger, C. Matthaeus, S. Kunz, A. von der Malsburg, F. Noé, A. Roux, M. van der Laan, W. Kühlbrandt, and O. Daumke. 2019. Structure and assembly of the mitochondrial membrane remodelling GTPase Mgm1. Nature. 571:429-433. doi:10.1038/s41586-0191372-3.

Ge, Y., X. Shi, S. Boopathy, J. McDonald, A.W. Smith, and L.H. Chao. 2020. Two forms of Opal cooperate to complete fusion of the mitochondrial inner-membrane. eLife. 9:e50973. doi:10.7554/eLife.50973.

Lee, H., S.B. Smith, S.-S. Sheu, and Y. Yoon. 2020. The short variant of optic atrophy 1 (OPA1) improves cell survival under oxidative stress. J. Biol. Chem. jbc.RA119.010983. doi:10.1074/jbc.RA119.010983.

Shi, H., A.J. Rampello, and S.E. Glynn. 2016. Engineered AAA+ proteases reveal principles of proteolysis at the mitochondrial inner membrane. Nat. Commun. 7. doi:10.1038/ncomms13301.

Takeyama, N., N. Matsuo, and T. Tanaka. 1993. Oxidative damage to mitochondria is mediated by the Ca2+-dependent inner-membrane permeability transition. Biochem. J. 294:719725. doi:10.1042/bj2940719. 